\title{
HOROCYCLIC COORDINATES FOR RIEMANN SURFACES \\ AND MODULI SPACES. I: TEICHMÜLLER AND RIEMANN SPACES OF KLEINIAN GROUPS
}

\author{
IRWIN KRA
}

Dedicated to Lipman Bers on the occasion of his seventy-fifth birthday

\section{TABLE OF CONTENTS}

0 . Introduction and statement of main results

1. Horocyclic coordinates

2. The $z w=t$ plumbing construction

3. The plumbing construction for an admissible graph

4. Deformation (Teichmüller) and moduli (Riemann) spaces

5. Torsion free terminal $b$-groups

6. One-dimensional deformation spaces

7. Deformation spaces for torsion free terminal $b$-groups

8. One-dimensional moduli spaces

9. Moduli spaces for torsion free terminal $b$-groups

10. Forgetful maps

11. Metrics on surfaces and their Teichmüller spaces

12. Appendix I: Calculations in $\operatorname{PSL}(2, \mathbb{C})$ and $\operatorname{SL}(2, \mathbb{C})$

13. Appendix II: A computer program for computing torsion free terminal $b$ groups

14. Appendix III: Independence of gluing on choice of annuli

\section{INTRODUCTION AND STATEMENT OF MAIN RESULTS}

This paper is concerned with the general problem of explicitly describing intrinsic parameters for Teichmüller and Riemann spaces. Ideally, we want to be able to read off from a given Riemann surface its position in moduli space. Further, we want to attach various geometric and analytic objects such

Received by the editors June 25, 1988; results from this paper were presented at the AMS-SIAMIMS Summer Research Conference on the geometry of Riemann surfaces and discrete groups, July 17, 1989.

1980 Mathematics Subject Classification (1985 Revision). Primary 32G15, 30F40.

Research partially supported by NSF grant DMS 8701774. This paper was revised and prepared for publication while the author was a Lady Davis Visiting Professor at the Hebrew University of Jerusalem during the fall of 1989.

(c) 1990 American Mathematical Society 
as uniformizations by Kleinian groups, meromorphic differentials, lengths of geodesics, etc., to the Riemann surface. These objects should be analytic functions of the moduli. This work is a contribution towards this general goal. At times our methods parallel those of Bers [B11], Earle-Marden [EM] and Wolpert [W1]. All of this development is based very significantly on the fundamental work of Maskit [Mt2]. We have profited greatly from reading these papers and from conversations with their authors. We will describe our main results after orienting the reader by discussing a classical example.

Every point $\tau$ in the upper half-plane $\mathbb{H}^{2}$ determines a rank 2 parabolic group $G_{\tau}$ generated by the motions $A: z \mapsto z+1$ and $B: z \mapsto z+\tau$ and a torus $T_{\tau}=\mathbb{C} / G_{\tau}$. Two such tori $T_{\tau_{1}}$ and $T_{\tau_{2}}$ are conformally equivalent if and only if there exists an $M \in \operatorname{PSL}(2, \mathbb{Z})$ with $M\left(\tau_{1}\right)=\tau_{2}$. The space $\mathbb{H}^{2}$ is the deformation or Teichmüller space $\mathbf{T}(1,0)$ for tori and $\mathbf{R}(1,0)=$ $\mathbb{H}^{2} / \operatorname{PSL}(2, \mathbb{Z})$ is its Riemann or moduli space. It is well known that $\mathbf{R}(1,0)$ is complex analytically equivalent to the sphere $\hat{\mathbb{C}}$ with three distinguished points: a Riemann surface of signature $(0,3 ; 2,3, \infty)$. To compactify $\mathbf{R}(1,0)$ one needs to add a single point at $\infty$. It is convenient to think of this ideal point as a singular torus obtained by pinching a curve to a point to produce a "node".

We now consider an alternate description of the quotient $T_{\tau}$. Start with the infinite cylinder $C=\mathbb{C} /\langle A\rangle$. Perfectly reasonable coordinates each vanishing at one end of the cylinder are $z=e^{2 \pi i \zeta}$ and $w=e^{-2 \pi i \zeta}, \zeta \in \mathbb{C}$. For each $t \in \mathbb{C}$ with $0<|t|<1$, we construct a torus $S_{t}$ by a "plumbing procedure". Remove from $C$ the two punctured discs $\{0<|z| \leq|t|\}$ and $\{0<|w| \leq|t|\}$ to obtain a finite cylinder $C^{\prime}$. Identify two points $P$ and $Q$ on $C^{\prime}$ if and only if $z(P) w(Q)=t$, and thus obtain a torus $S_{t}$ together with a "central curve" described in local coordinates by $\{|z|=1\}=\{|w|=1\}$. It is easy to see that we have obtained surfaces that satisfy $S_{e^{2 \pi i \tau}}=T_{\tau}$ for all $\tau \in \mathbb{H}^{2}$. This construction can be extended to $t=0$ to obtain a singular surface $S_{0}$ : a torus with a node.

The punctured unit disc (the set of $t$ coordinates) is a covering of the moduli space for tori; it corresponds to $\mathbf{T}(1,0) / \mathbb{Z}$, where $\mathbb{Z}$ acts on $\mathbb{H}^{2}=\mathbf{T}(1,0)$ by translation and the generator of $\mathbb{Z}$ acts on the torus as the Dehn twist about the central curve (changing $\tau$ to $\tau+1$ ). The addition of the origin gives a partial compactification of $\mathbf{T}(1,0) / \mathbb{Z}$.

If we normalize the metric (of zero curvature) so that $S_{t}$ has area 1 , then the length of the central curve is $\sqrt{-2 \pi / \log |t|}$. Thus the limiting surface corresponding to $t=0$ is obtained by shrinking the central curve on $S_{t}$ to obtain a node. (On $S_{t}$, there is also a geodesic "transverse" to the central curve of length

$$
\frac{|\log t|}{2 \pi} \sqrt{\frac{-2 \pi}{\log |t|}} .
$$

On the limiting surface $S_{0}$, the transverse curves have infinite length.) 
The above construction and analysis is carried over, in this paper, to the case of Riemann surfaces of finite analytic type and constant negative curvature. To begin, we describe the topological data.

Let $\mathscr{G}$ be an "admissible graph" (see $\S 3.2)$ of type $(p, n)$ with $d=3 p-3+n$ $(\geq 0)$ edges and $v=2 p-2+n(>0)$ vertices. We associate to each vertex of $\mathscr{G}$ a "pair of pants" (a sphere with three disjoint open discs removed) and to each edge "a tube for a plumbing construction" to connect two boundary components of the same or different pairs of pants. The edges also determine central curves on the tubes. In this manner, the graph $\mathscr{G}$ determines a nonsingular topological surface $S$ of type (genus $p, n$ punctures) together with a maximal partition $\Sigma$ of the surface into parts. The edges of $\mathscr{G}$ are in one-to-one correspondence with the partition ( $=$ central) curves in $\Sigma$. We shall denote by $a_{k}$ both the $k$ th edge on $\mathscr{G}$ and the partition curve on $S$ that it determines. Each partition curve $a_{k}$ also determines a subsurface $T_{k}$ of $S$ of type $(0,4)$ or $(1,1)$, known as a "modular part" of $S$ (see $\S 5.3$ ): it is the component containing $a_{k}$ of the surface obtained by cutting $S$ along all partition curves in $\Sigma$ except $a_{k}$. We can consider surfaces $S$ with singularities by shrinking (pinching) some or all of the partition curves in $\Sigma$ to nodes. This data specifies the topological construction of a surface from thrice punctured spheres.

For the construction of Riemann surfaces (perhaps with nodes), we introduce complex coordinates (numbers assigned to each edge in $\mathscr{G}$ ). We begin with some standard notation. For $r \in \mathbb{R}^{+}$,

$$
\Delta_{r}=\{z \in \mathbb{C} ;|z|<r\} \text { and } \Delta=\Delta_{1} .
$$

For $t \in \mathbb{C}^{d}, t=\left(t_{1}, \ldots, t_{d}\right)$, we set

$$
|t|=\max \left\{\left|t_{j}\right| ; j=1, \ldots, d\right\} \text {. }
$$

Using a graph $\mathscr{G}$ and "complex coordinates" $t$, with $t_{k}$ associated to the edge $a_{k}$, one constructs a Riemann surface $S_{t}$ using plumbing operations (see $\S \S 2.3$ and 3.4). The region (in $\mathbb{C}^{d}$ ) for which the construction is valid can only be described qualitatively; its exact shape, for example, is not known. A subregion of the resulting coordinate space corresponds to Riemann surfaces obtained by a particularly simple form of the construction. These simple plumbing constructions we call "tame". In tame plumbing we glue horocircles to horocircles on the thrice punctured spheres (and other building blocks); the general plumbing operation replaces the horocircles by arbitrary Jordan curves.

Theorem 1 (see also [EM]). Fix a graph $\mathscr{G}$ of type $(p, n)$.

(a) For $t \in \mathbb{C}^{d}$ with $|t|<e^{-2 \pi}$, there is a canonically (depending on $\mathscr{G}$ and $t$ ) constructed Riemann surface $S_{t}$. The construction consists of $d$ tame plumbing operations. These Riemann surfaces fill out an open set in the compactification of the moduli space $\mathbf{R}(p, n)$.

(b) There exists a simply connected domain of holomorphy $\mathbf{D}(\mathscr{G}),\left(\Delta_{e^{-2 \pi}}\right)^{d} \subset$ $\mathbf{D}(\mathscr{G}) \subset\left(\Delta_{e^{-\pi / 2}}\right)^{d}$, such that every Riemann surface of type $(p, n)$ corresponds 
to (in general, infinitely many) points $t \in \mathbf{D}(\mathscr{G})$ via the the (general) plumbing construction.

The parameters $t$ described by the above theorem are the horocyclic coordinates for moduli spaces referred to in the title of this paper. The surface $S_{t}$ is nonsingular whenever all the $t_{j}$ are nonzero. The zero components of $t$ correspond precisely to the nodes of $S_{t}$. To describe the construction, start with $v$ (thrice punctured) spheres, one for each vertex of $\mathscr{G}$. If two vertices of $\mathscr{G}$ are joined by an edge $a_{k}$, plumb the associated spheres using the parameter $t_{k}$. On the resulting surface $S_{t}$, the edge $a_{k}$ is replaced by a tube with a partition curve or node. The complement of the set of nodes and partition curves is a disjoint union of $v$ pairs of pants; these are the parts of $S_{t}$. The interpretation of the plumbing construction in a Kleinian group setting leads to the following uniformization

Theorem 2. For each $t \in \mathbf{D}(\mathscr{G})$ with $t_{j} \neq 0, j=1, \ldots, d$, we construct a unique torsion free terminal b-group $\Gamma_{t} \subset \operatorname{PSL}(2, \mathbb{C})$ that represents $S_{t}$ (see $\S 5.1)$. The generators for $\Gamma_{\text {, }}$ are represented by elements of $\operatorname{SL}(2, \mathbb{C})$ whose entries are rational functions of $\log t_{j}, j=1, \ldots, d$.

Theorem 3 (see also [EM, B11, Mt3]). The graph $\mathscr{G}$ determines global complex analytic coordinates ${ }^{1} \tau=\left(\tau_{1}, \ldots, \tau_{d}\right)$ on the Teichmüller space $\mathbf{T}(p, n)$ with the following properties:

(a) We have the inclusions

$$
U^{(1)} \times \cdots \times U^{(d)} \supset \tau(\mathbf{T}(p, n)) \supset\left(U_{2}\right)^{d},
$$

where $U_{k}=\{z \in \mathbb{C} ; \operatorname{Im} z>k\}, k \in \mathbb{R}^{+}, U^{(j)}=U_{1 / 2}$ if the modular subsurface $T_{j}$ corresponding to the jth edge in $\mathscr{G}$ is a four times punctured sphere, and $U_{1}$ otherwise (the edge corresponds to a punctured torus).

(b) The Dehn twist about the curve $a_{k}$ corresponding to the kth edge in $\mathscr{G}$ is given by the restriction to $\tau(\mathbf{T}(p, n))$ of the translation of $\mathbb{C}^{d}$ by the vector $2(0, \ldots, 0,1,0, \ldots, 0)$ (where the one is in the kth spot).

(c) Let $c:[0,1) \rightarrow \mathbf{T}(p, n)$ be a continuous path. For $0 \leq s<1$, let $l_{k}(s)$ be the hyperbolic length on the marked Riemann surface $c(s)$ of the unique geodesic freely homotopic to the curve $a_{k}$. Then $\lim _{s \rightarrow 1} \operatorname{Im} \tau_{k}(s)=\infty$, whenever $\lim _{s \rightarrow 1} l_{k}(s)=0$.

(Here $\tau_{k}(s)$ is the $k$ th component of $c(s)$.)

Before describing a converse to part (c) of the above theorem, we discuss the nature of our coordinates and introduce some forgetful maps. We use the horocylic coordinates to identify $\mathrm{T}(p, n)$ with an open subset of $\mathbb{C}^{d}$. Let $J$ be a subset of $\{1,2, \ldots, d\}=\mathbb{Z}_{d}$. Consider the topological surface with nodes

\footnotetext{
${ }^{1}$ These coordinates are essentially canonical. The $t$-coordinates are uniquely determined up to some signs by $\mathscr{G}$ and an ordering on its edges. To obtain the $\tau$ 's one must choose branches of the logarithms of the $t$ 's. Thus each $\tau_{j}$ is uniquely determined by $\mathscr{G}$ modulo $\mathbb{Z}$.
} 
obtained from $S$ by shrinking each curve $a_{j}$, with $j \in J$, to a node. Call the resulting surface $S_{J}$. The curves $a_{k}, k \in \mathbb{Z}_{d}-J$, form a maximal partition on each of the parts of $S_{J}$. We identify $\mathbb{C}^{d-|J|}$ with the subspace $\left\{t \in \mathbb{C}^{d} ; t_{j}=0\right.$ for $j \in J\}$. Let $\rho_{J}$ be the projection of $\mathbb{C}^{d}$ onto $\mathbb{C}^{d-|J|}$ and $\mathbf{T}_{J}(p, n)$ be the image of $\mathbf{T}(p, n)$ under $\rho_{J}$. As a consequence of an isomorphism theorem due to Maskit [Mt4], $\mathbf{T}_{J}(p, n)$ is biholomorphic to the product of the Teichmüller spaces of the parts of $S_{J}$.

We are now ready to state the converse to Theorem 3 (c).

Theorem 3. (d) In addition to the hypothesis of Theorem 3(c), also assume that $\lim _{s \rightarrow 1} \operatorname{Im} \tau_{k}(s)=\infty, k \in J$, and that $\lim _{s \rightarrow 1} \rho_{J}(c(s))$ exists (in $\left.\mathbf{T}_{J}(p, n)\right)$. Then $\lim _{s \rightarrow 1} l_{k}(s)=0$, all $k \in J$.

Thus we have that for $k \in J, \operatorname{Im} \tau_{k}$ tends to plus infinity if and only if the hyperbolic length of the geodesic freely homotopic to $a_{k}$ tends to zero provided the remaining horocyclic coordinates converge.

To see the relation between Theorems 1 and 3 , let $\mathbf{D}_{0}(\mathscr{G})$ consist of those points in $\mathbf{D}(\mathscr{G})$ with all coordinates nonzero. Then $\mathbf{D}_{0}(\mathscr{G})$ is precisely

$$
\tau(\mathbf{T}(p, n)) /(2 \mathbb{Z})^{d}
$$

where the generators of $(2 \mathbb{Z})^{d}$ are the Dehn twists about the partition curves.

Theorem 4. The group of automorphisms of $\mathscr{G}$, Aut $\mathscr{G}$, acts as a group of complex analytic self-maps of $\mathbf{D}(\mathscr{G})$ that preserves $\mathbf{D}_{0}(\mathscr{G})$. The quotient space $\mathbf{D}(\mathscr{G}) /$ Aut $\mathscr{G}$ is a complex orbifold. The quotient $\mathbf{D}_{0}(\mathscr{G}) /$ Aut $\mathscr{G}$ represents conjugacy classes of terminal regular b-groups determined by the graph $\mathscr{G}$.

A road map to the proofs of the theorems of the introduction is as follows. Theorem 1(a) is to be found in $\S 3.5$; the estimates for part (b) and Theorem 3 (a) appear in $\S \S 6.1$ and 6.3. Theorem 2 is proven in $\S 7.5$. The horocyclic coordinates for Theorem 3 appear in $\S 7.2$. Theorem $3(\mathrm{~b})$ is in $\S 7.4$, while parts (c) and (d) are proven in $\S 11.6$. Theorem 4 is established in $\S \S 9.4$ and 9.7.

This paper is intended to be the first of a series; in subsequent parts we shall study

II: The strong deformation spaces of Bers, and

III: Cusp forms for terminal $b$-groups.

We end the introduction with a few more remarks about motivation and a brief description of the historical background. The main motive is to find good complex analytic coordinates for moduli spaces. There are several reasons for continuing to seek such coordinates. Good coordinates for Teichmüller spaces should be intrinsic, and one should be able to determine the relationships between various sets of such coordinates. We are also interested in obtaining coordinates that extend to the "points at infinity" of moduli spaces; these points correspond to surfaces with nodes. The earliest complex coordinates for Teichmüller spaces involve periods of abelian differentials (Ahlfors [A1, 1960] 
and Rauch [Ra, 1960]) and Schwarzian derivatives of univalent maps (Bers [B1, 1958; B4, 1966]). Special classes of Kleinian groups produce coordinates for $\mathrm{T}(p, n)$; these have been investigated by Maskit [Mt3, 1974], Earle [E, 1981], Kra-Maskit [KM1, 1981; KM2, 1982], Kra [K6, 1988].

Beginning in the early 1970s Lipman Bers as well as Clifford Earle and Albert Marden (joint work) began to study coordinates for compactified moduli space as well as the plumbing constructions known much earlier to algebraic geometers. ${ }^{2}$ Earle-Marden were probably among the first complex analysts to use plumbing constructions; they discussed plumbings during the 1972/73 special year at Mittag-Leffler. Their results were alluded to in expository papers [Mn1, 1977; Mn2, 1980] and described in a recent research announcement [Mn3, 1987] by Marden. Bers introduced strong deformation spaces and new uses for Fenchel-Nielsen coordinates at the 1973 Maryland Conference; his results were announced without proofs in [B7, 1974; B8, 1974; B9, 1975; B11, 1981]. Earle and Marden outlined their methods at talks at the Hawaii Conference in $1979^{3}$ and at Oberwolfach 1981; the proofs of their results are not in print except for the partial preprint [EM, $\geq 1989]$. Although Maskit [Mt3, 1974] was mainly interested in coordinates for $\mathbf{T}(p, n)$, his methods lead as well to coordinates at infinity. In reviewing the literature on this subject one must also mention Fay's interesting book [F, 1973] although it approaches the subject from a more algebraic-geometric point of view. It should be remarked that the compactification of moduli space using horocyclic coordinates yields the same complex orbifold as the one studied by the algebraic geometers (for example [DM]). Clearly, a tremendous amount of work had been done in this area by early 1988. This body of work was used by many authors explicitly (for example, Masur [Mr, 1976], Earle-Kra [EK2, 1986], Wolpert [W1, 1983; W2, $\geq 1989$ ], Earle-Sipe [ES, $\geq 1989]$ ) and Hejhal [H2, $\geq 1989]$ ) despite the fact that most of the results and almost all proofs had not appeared in print. There was also speculation (conjectured by Bers) that the various approaches discussed above lead to essentially the same coordinates.

My interest in the subject originated with an attempt to understand the different methods of compactifying moduli space and to obtain geometric coordinates for $\mathbf{T}(p, n)$ [K6, 1988]. I used generalizations of the groups first considered by Maskit [Mt3] to obtain coordinates. These are terminal, regular $b$-groups. Bers [B11] and Earle-Marden [EM] do not use b-groups; we shall discuss the exact relations between these three approaches in the second of this series of papers.

Part (a) of Theorem 1 appears in [EM]. The constants in part (b) are new. The Earle-Marden construction emphasizes the use of arbitrary coordinates on thrice punctured spheres. They thus obtain more general local coordinates on

\footnotetext{
${ }^{2}$ I have been unable to trace the historical origins of the $z w=t$ construction. I learned it from Marden and Wolpert (especially from the latter's 1987 Helsinki lecture). A special feature of the current work is the use of the rigid nature of this construction, if one restricts to special coordinates $z$ and $w$.

${ }^{3}$ An abstract of Marden's talk was distributer at this conference.
} 
$\mathbf{D}(\mathscr{G})$. In our approach, we use only horocyclic coordinates on spheres, thus leading to a general definition of $\mathbf{D}(\mathscr{G})$ (see $\$ 3.5$ ) that does not require analytic continuation. Theorem 2 is new and one of the main results of this investigation: the terminal $b$-group uniformizing the surface is a rational function of the logarithms of the plumbing parameters. This theorem will allow us to explore the relations between the constructions of this paper, and those of Maskit [Mt3], Bers [B11] and Earle-Marden [EM]. We will show as a result of Theorem 2, in part II, that the approaches of Bers, Earle-Marden, Maskit and this author to compactifying moduli space are essentially the same. Theorem 3 appears in [EM and Mt3]. A result of this type can also be derived from the ideas of [B11]. This paper contains the first proof of the existence of coordinates on $\mathbf{T}(p, n)$ having properties of parts (b), (c) and (d). Theorem 4 is new, but such a result can be derived from the methods of [EM, Mt3 or B11].

We have included in this paper, for the convenience of the reader, those results that are well known to the experts but are nevertheless absent from the literature. Our exposition of these topics includes material not previously formulated. We hope that the leisurely approach that we have taken will be of benefit to the reader. It is obvious that, in portions of this manuscript, I am reproving results obtained (but not published) by Earle-Marden and Bers. I hope that I have properly attributed credit to their work. This task is complicated because the evolving nature of the theorems and approaches in the research announcements makes it difficult to determine who knew what at which moment in time. It is my pleasure to thank the referee for a careful reading of this manuscript and for the many helpful suggestions.

Notation. $\mathbb{Z}, \mathbb{Q}, \mathbb{R}, \mathbb{C}:$ the integers, rationals, reals, complexes.

$\mathbb{Z}^{+}, \mathbb{R}^{+}:$the positive integers, reals.

$\hat{\mathbb{C}}=\mathbb{C} \cup\{\infty\}$ : the extended complex plane (similarly, $\hat{\mathbb{Q}}, \hat{\mathbb{R}}$ ).

$\mathbb{C}^{d}=($ complex $)$ euclidean $d$-dimensional space.

$\mathbb{H}^{2}=$ upper half-plane $=\{\zeta \in \mathbb{C} ; \operatorname{Im} \zeta>0\}$.

$\mathbb{H}^{2}=$ lower half-plane $=\{\zeta \in \mathbb{C} ; \operatorname{Im} \zeta<0\}$.

$\operatorname{SL}(2, \mathbb{C}): 2 \times 2$ complex matrices of determinant 1 .

$\operatorname{PSL}(2, \mathbb{C})=\operatorname{SL}(2, \mathbb{C}) / \pm I$ acting on $\hat{\mathbb{C}}$ via Möbius transformations.

$\operatorname{SL}(2, \mathbb{R}), \operatorname{PSL}(2, \mathbb{R}), \operatorname{SL}(2, \mathbb{Z}), \operatorname{PSL}(2, \mathbb{Z})$ : obvious subgroups of above two groups.

$\operatorname{PGL}(2, \mathbb{Z}): 2 \times 2$ integral matrices with determinant \pm 1 , moduli $\pm I$.

$A=\left[\begin{array}{ll}a & b \\ c d\end{array}\right] \in \mathrm{SL}(2, \mathbb{C}), \operatorname{tr} A=(a+d)$.

For parabolic $A \in \mathrm{SL}(2, \mathbb{C}), f(A)=$ fixed point of (the Möbius transformation) $A$.

$[A, B]=A^{-1} \circ B^{-1} \circ A \circ B=$ commutator of the elements $A$ and $B$ of $\operatorname{SL}(2, \mathbb{C})$.

$\operatorname{cr}(\zeta, a, b, c)=\frac{\zeta-b}{\zeta-a} \frac{c-a}{c-b}$ (a fixed cross ratio function of four distinct points in $\hat{\mathbb{C}})$.

$\Gamma=$ torsion free (usually) terminal $b$-group (see $\S 5.1$ ). 
$\Delta=\Delta(\Gamma)=$ invariant component of $\Gamma$.

$\Omega=\Omega(\Gamma)=$ region of discontinuity of $\Gamma$.

$\Lambda=\Lambda(\Gamma)=$ limit set of $\Gamma$.

$N(\Gamma)=$ normalizer of $\Gamma$ in $\operatorname{PSL}(2, \mathbb{C})$.

$N_{\mathrm{qc}}(\Gamma)=$ normalizer of $\Gamma$ in the group of quasiconformal automorphisms of $\hat{\mathbb{C}}$.

$\mathscr{G}=$ augmented admissible graph (see $\S 3.2$ ).

$(p, n)=$ type of $b$-group $\Gamma$ or of graph $\mathscr{G}$.

$d=3 p-3+n=d(\mathscr{G})$.

$v=2 p-2+n=v(\mathscr{G})$.

$\mathbf{T}(\Gamma)=$ Teichmüller or deformation space of $\Gamma$ (see $\S 4.1)$.

$\mathbf{V}(\Gamma)=$ punctured Teichmüller curve of $\Gamma$ (see $\S 4.6)$.

Mod $\Gamma=$ modular group of $\Gamma$ (see $\S 4.2)$.

$\mathbf{R}(\Gamma)=$ Riemann or moduli space of $\Gamma$ (see $\S 4.2)$.

$\mathbf{T}(p, n)=$ Teichmüller space of Riemann surfaces of type $(p, n)$. $\S 3.5)$.

$\mathbf{D}(\mathscr{G})=$ Teichmüller (or deformation) space corresponding to graph $\mathscr{G}$ (see

$\mathbf{D}_{0}(\mathscr{G})=$ points in $\mathbf{D}(\mathscr{G})$ representing nonsingular surfaces (see $\S 3.8$ ).

$\mathbf{V}(\mathscr{G})=$ curve over $\mathbf{D}(\mathscr{G})$ (see $\S 3.10)$.

$\mathbf{R}(\mathscr{G})=$ Riemann space corresponding to graph $\mathscr{G}$ (see $\S 9.7$ ).

$F(a, b, c)=$ triangle group defined in $\S 12.1$.

\section{HOROCYCLIC COORDINATES}

This section contains the basic facts about horocyclic coordinates on the thrice punctured sphere $S$ as well as convenient ways to choose generators for covering groups of $S$. These groups are the basic building blocks for the constructions of terminal $b$-groups in $\S \S 5,6$ and 7 (via the Klein-Maskit [Mt3] combination theorems).

1.1. We start with some useful language.

(A) A horocycle or horocircle $L$ for a parabolic Möbius transformation $C$ with fixed point $c$ is a circle through $c$ invariant under $C$. We shall consider the horocircle to be oriented so that for all $z \in L-\{c\}$, the three points $z$, $C(z)$, and $C^{2}(z)$ follow each other in the positive orientation. By abuse of language we shall also call $L-\{c\}$ a horocircle. The interior of a horocircle is called a horodisc.

(B) Let $\Gamma$ be a group of Möbius transformations and $G$ a subgroup of $\Gamma$. Let $X$ be a subset of $\hat{\mathbb{C}}$. We say that $X$ is precisely invariant under $G$ in $\Gamma$ (Maskit [Mt3]) if $\gamma(X)=X$ for all $\gamma \in G$ and $\gamma(X)$ is disjoint from $X$ for all $\gamma \in \Gamma-G$.

(C) A triangle group is a Kleinian group $\Gamma$ with invariant component $\Delta$ such that $\Delta / \Gamma$ has signature $\left(0,3 ; \nu_{1}, \nu_{2}, \nu_{3}\right)$ with $\nu_{1}^{-1}+\nu_{2}^{-1}+\nu_{3}^{-1}<1$. It is well known (see, for example $[\mathrm{K} 1]$ ) that $\Delta$ is a disc in $\hat{\mathbb{C}}$ and hence two triangle groups are conjugate in $\operatorname{PSL}(2, \mathbb{C})$ if and only if they have the same signature. 


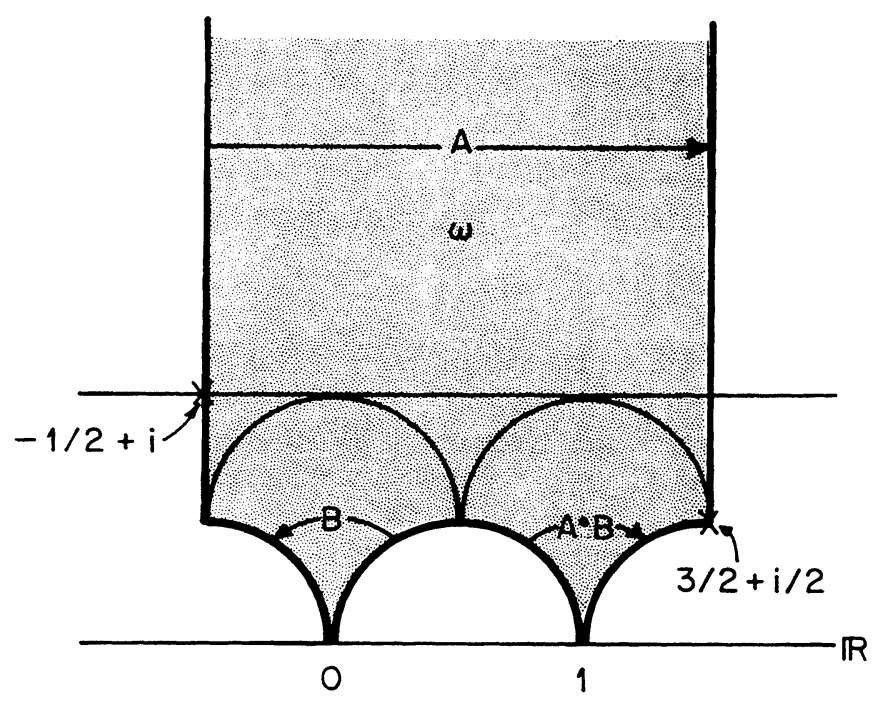

FIGURE 1. The fundamental domain in $\mathbb{H}^{2}$ for triangle group $F=F(\infty, 0,1)$, showing the largest disjoint horodiscs of the same radii $\left(e^{-\pi}\right)$.

We shall make extensive use of torsion free triangle groups (groups of signature $(0,3 ; \infty, \infty, \infty))$. A notation for describing such groups will be found in $\S 12$.

1.2. Let $S$ be the thrice punctured sphere:

$$
S=\hat{\mathbb{C}}-\left\{P^{1}, P^{2}, P^{3}\right\},
$$

where $P^{j} \in \hat{\mathbb{C}}$ for $j=1,2,3$. Let $\rho: \mathbb{H}^{2} \rightarrow S$ be a holomorphic universal covering map. Let $F$ be the covering group of $\rho$. By conjugation, we may take $F$ to be the level 2 principal congruence subgroup of $\operatorname{PSL}(2, \mathbb{Z})$; that is,

$$
F=\{\gamma \in \operatorname{PSL}(2, \mathbb{Z}) ; \gamma \equiv I(\bmod 2)\} .
$$

The group $F$ is generated by two parabolic motions

$$
A=\left[\begin{array}{cc}
-1 & -2 \\
0 & -1
\end{array}\right], \quad B=\left[\begin{array}{cc}
-1 & 0 \\
2 & -1
\end{array}\right]
$$

with

$$
A \circ B=\left[\begin{array}{ll}
-3 & 2 \\
-2 & 1
\end{array}\right]
$$

also parabolic. Every parabolic element of $F$ is conjugate to a power of one of the above three elements. A fundamental domain $\omega$ for the action of $F$ on $\mathbb{H}^{2}$ is shown in Figure 1. 
The positively oriented straight lines $\left\{x+i y_{0} ; x \in \mathbb{R}\right\}$ with fixed $y_{0} \in \mathbb{R}$ are the horocircles for $A$ and $\mathbb{H}^{2}+\frac{1}{2} i=\left\{z \in \mathbb{C} ; \operatorname{Im} z>\frac{1}{2}\right\}$ is precisely invariant under the cyclic subgroup $\langle A\rangle$ in $F$.

The map $\rho$ extends continuously to the parabolic fixed points of $F$ (that is, to $\hat{\mathbb{Q}} \subset \hat{\mathbb{R}}=\partial \mathbb{H}^{2}$ ) and it involves no loss of generality to assume that

$$
\rho(\infty)=P^{1}, \quad \rho(0)=P^{2}, \quad \rho(1)=P^{3} .
$$

The map $\rho$ induces a canonical correspondence between conjugacy classes of maximal parabolic cyclic subgroups of $F$ and the punctures of $S$. Thus the punctures $P^{1}, P^{2}, P^{3}$ on $S$ are determined by (or correspond to) the primitive parabolic elements $A, B,(A \circ B)^{-1}$ of $F$.

1.3. Let $\Gamma$ be any torsion free triangle group. Two parabolic elements $A$ and $B$ are called canonical generators for $\Gamma$ if they generate $\Gamma$ and if $A \circ B$ is also parabolic. The generators of $F$ (of $\S 1.2$ ) given by (1.2.2) are obviously canonical generators. Clearly, any primitive parabolic element of $\Gamma$ can appear as one of a pair of canonical generators, and each generator must be a primitive element of $\Gamma$.

Lemma. Let $\Gamma$ be a torsion free triangle group. Let $(A, C)$ and $\left(A, C_{1}\right)$ be two sets of canonical generators for $\Gamma$. Then there exists an $n \in \mathbb{Z}$ with $C_{1}=$ $A^{n / 2} \circ C \circ A^{-n / 2}$.

Proof. Without loss of generality $\Gamma=F$, the triangle group described in $\S 1.2$, and $A$ is given by (1.2.2). Choose a lift of $C$ to $\operatorname{SL}(2, \mathbb{C})$ with $\operatorname{tr} C=-2$;

$$
C=\left[\begin{array}{cc}
a & b \\
c & -2-a
\end{array}\right], \quad-2 a-a^{2}-b c=1,
$$

where $a$ is an odd integer and $b, c$ are even integers. Since $A \circ C$ is parabolic, $\operatorname{tr}(A \circ C)= \pm 2$. If $\operatorname{tr}(A \circ C)=2$, then $c=0$ and $\Gamma$ is elementary (see also [K5]). Thus $\operatorname{tr}(A \circ C)=-2$ and it follows that $(A, C)$ is a canonical pair of generators for $F$ if and only if

$$
C=\left[\begin{array}{cc}
a & -\frac{1}{2}(1+a)^{2} \\
2 & -2-a
\end{array}\right], \quad a \in \mathbb{Z}, a \equiv 1(\bmod 2) .
$$

The fixed point $f(C)$ is $\frac{1}{2}(1+a) \in \mathbb{Z}$. Take $B$ of (1.2.1). It follows that

$$
C=A^{n / 2} \circ B \circ A^{-n / 2}, \quad n=\frac{1}{2}(1+a)=\text { fixed point of } C .
$$

Remark. Note that if $(A, C)$ is a canonical pair of generators for $\Gamma$ then $\left(A, C^{-1}\right)$ is not canonical; that is, $A \circ C^{-1}$ must be hyperbolic. We also observe that for a pair $(A, C)$ of canonical generators for $\Gamma, A \circ C=A^{1 / 2} \circ C^{-1} \circ A^{-1 / 2}$ and hence $f(A \circ C)=A^{1 / 2}(f(C))$.

Let $\Gamma$ be a torsion free triangle group. Two pairs $\left(A_{1}, B_{1}\right)$ and $\left(A_{2}, B_{2}\right)$ of canonical generators for $\Gamma$ are equivalent provided $A_{1}$ is conjugate to $A_{2}^{ \pm 1}$ and $B_{1}$ is conjugate to $B_{2}^{ \pm 1}$ in $\Gamma$. Choose an invariant component $\Delta$ of $\Gamma$ 
and a covering map $\rho$ of $S$ by $\Delta$ with covering group $\Gamma$. Then $\left(A_{1}, B_{1}\right)$ is equivalent to $\left(A_{2}, B_{2}\right)$ if and only if $\rho\left(f\left(A_{1}\right)\right)=\rho\left(f\left(A_{2}\right)\right)$ and $\rho\left(f\left(B_{1}\right)\right)=$ $\rho\left(f\left(B_{2}\right)\right)$.

Corollary. Let $(A, B)$ be canonical generators for the torsion free triangle group $\Gamma$. The most general pair of canonical generators for $\Gamma$ equivalent to $(A, B)$ are $\left(C \circ A \circ C^{-1}, C \circ A^{n} \circ B \circ A^{-n} \circ C^{-1}\right)$ and $\left(C \circ A^{-1} \circ C^{-1}, C \circ A^{n} \circ B^{-1} \circ A^{-n} \circ C^{-1}\right)$ with $n \in \mathbb{Z}$ and $C \in \Gamma$.

Remarks. (1) Let $\Gamma$ be a torsion free triangle group with invariant components $\Delta$ and $\Delta^{*}$. Let $(A, B)$ be canonical generators for $\Gamma$. We observe that the fixed points of $A, B$ and $B^{-1} \circ A^{-1}$ always lie on the same side of the respective horocircles determined by these elements on a given component. The fixed points lie to the left of the horocircles on $\Delta$ if and only if they lie to the right of the horocircles on $\Delta^{*}$.

(2) Let $\left(A_{j}, B_{j}\right)$ be canonical generators for the triangle group $F_{j}, j=$ 1, 2. There exists a unique $C \in \operatorname{PSL}(2, \mathbb{C})$ such that $C \circ A_{1} \circ C^{-1}=A_{2}$, $C \circ B_{1} \circ C^{-1}=B_{2}$ and $C F_{1} C^{-1}=F_{2}$. See $\S 12.1$

1.4. Proposition. Consider the thrice punctured sphere $S$ endowed with the Poincaré metric $d$ of constant negative curvature -1 . Let $P^{1}$ and $P^{2}$ be two distinct punctures on $S$. There exists on $S$ a unique simple geodesic $c=c\left(P^{1}, P^{2}\right), c: \mathbb{R} \rightarrow S$, such that for the arc length parametrization $c(s)$,

$$
\lim _{s \rightarrow \infty} c(s)=P^{1}, \quad \lim _{s \rightarrow-\infty} c(s)=P^{2} .
$$

Proof. We use the notation of $\S 1.2$. Existence of $c$ is trivial. Set

$$
c(s)=\rho\left(i e^{s}\right), \quad s \in \mathbb{R} .
$$

Property (1.4.1) holds and

$$
d\left(c\left(s_{1}\right), c\left(s_{2}\right)\right)=\left|s_{1}-s_{2}\right|, \quad \text { all } s_{1}, s_{2} \in \mathbb{R} .
$$

Let $\tilde{c}$ be a simple geodesic that satisfies (1.4.1) (see Figure 2, p. 510). The lift of $\tilde{c}$ may be chosen as a straight line in $\mathbb{H}^{2}$, perpendicular to $\mathbb{R}$. Its end point is the fixed point of a generator $B_{1}$ of $F$ with the property that $B_{1}$ is conjugate to $B$ in $F$ and $\left(A, B_{1}\right)$ is a canonical pair of generators. Now by Lemma 1.3, $B_{1}=A^{n / 2} \circ B \circ A^{-n / 2}$ with $n \in \mathbb{Z}$. But $n$ must be even because $A^{1 / 2} \circ B \circ A^{-1 / 2}=(A \circ B)^{-1}$ is not conjugate in $F$ to $B$. Thus $\tilde{c}=c$.

Remark. The thrice punctured sphere $S=\mathbb{H}^{2} / F$ has a unique anticonformal involution $J$ fixing the three punctures; $J$ is induced by the self-map of $\mathbb{H}^{2}$ given by $z \mapsto-\bar{z}$. The fixed curves of $J$ are precisely the three geodesics described by the proposition. A symmetric fundamental domain for $F$, with lifts for the three geodesic curves, is shown in Figure 3 (see p. 511). 

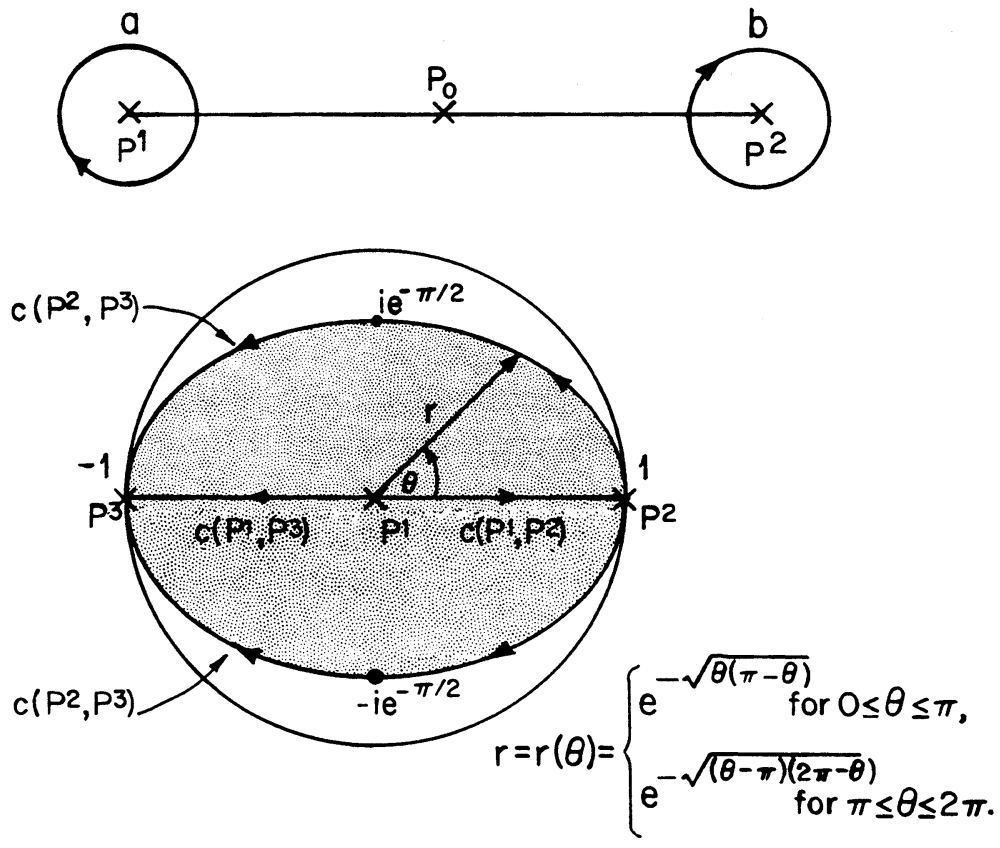

FIGURE 2. Canonical generators determined by a simple geodesic between punctures on a thrice punctured sphere and a target domain of a horocyclic coordinate at $P^{1}$ relative to $P^{2}$.

1.5. We now use the geodesics defined above to introduce distinguished coordinates at the punctures of thrice punctured spheres. Assume the situation of Proposition 1.4 and let $P^{3}$ be the third puncture on $S$. We define a function $f$ on a deleted neighborhood $N$ of $P^{1}$ by

$$
f(z)=e^{\pi i \rho^{-1}(z)}, \quad z \in N,
$$

where we choose the branch of $\rho^{-1}$ so that $f$ maps the portion of $c=$ $c\left(P^{1}, P^{2}\right)$ inside $N$ into the open unit interval $I=\{z \in \mathbb{C} ; 0<\operatorname{Re} z<1$, $\operatorname{Im} z=0\}$. The function $f$ has a holomorphic extension to the origin of $N$ (the point $P^{1}$ ) that satisfies $f(0)=0$. The germ of $f$ can be continued analytically along all curves in $S \cup\left\{P^{1}\right\}$. Thus the germ of $f$ defines a holomorphic function on each simply connected domain $D$ in $S \cup\left\{P^{1}\right\}$. The resulting function is necessarily injective. A maximal domain of definition for this function consists of the complement in $\hat{\mathbb{C}}$ of a simple curve from $P^{2}$ to $P^{3}$. In particular, we now let $f$ be our function defined on

$$
D=S \cup\left\{P^{1}\right\}-c\left(P^{2}, P^{3}\right) .
$$

Thus we have $f\left(P^{1}\right)=0, f(c)=I$ and $\lim _{s \rightarrow-\infty} f(c(s))=1$. Now if $x_{j}=$ 


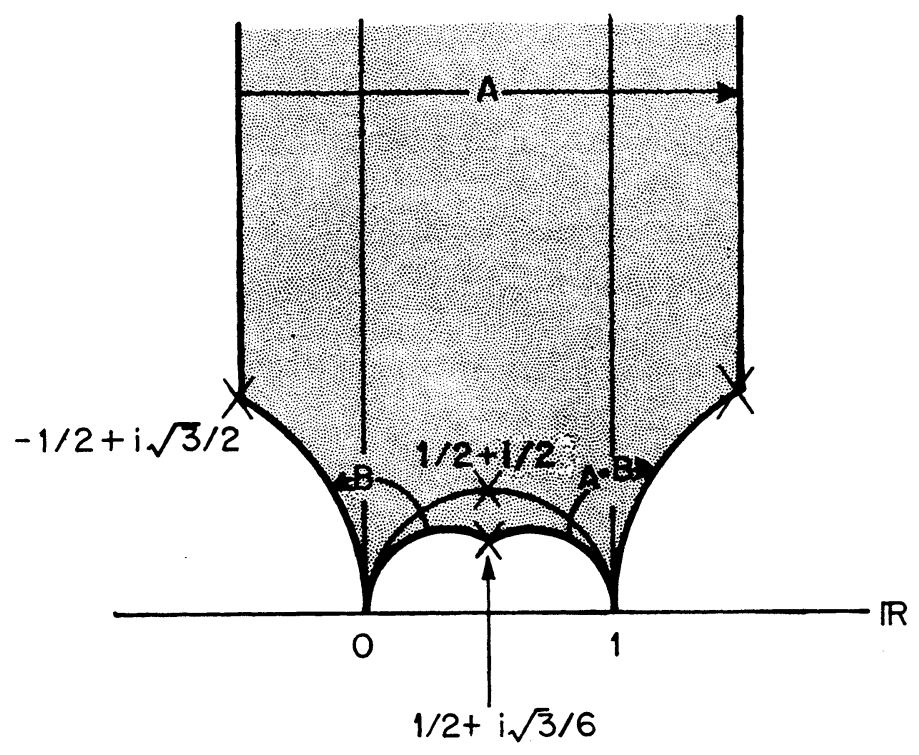

FIGURE 3. The three geodesics determined by the punctures on a surface of type $(0,3)$.

$c\left(s_{j}\right), j=1,2$, then from (1.4.3) and (1.5.1) we see that

$$
d\left(x_{1}, x_{2}\right)=\left|s_{1}-s_{2}\right|=\left|\log \left(\frac{\log f\left(x_{1}\right)}{\log f\left(x_{2}\right)}\right)\right| .
$$

Further, the formula for $f$ shows that it defines an isometry from $D-\left\{P^{1}\right\}$ with metric $d$ (the restriction of the Poincare metric on $S$ of curvature -1) into the punctured unit disc $\{0<|w|<1\}$ with its hyperbolic metric (ds= $-|d w| /|w| \log |w|)$. As a matter of fact, this property characterizes $f$.

Proposition and definition. Let $g$ be the germ of a holomorphic local homeomorphism defined in a neighborhood $N_{0}$ of $P^{1} \in S \cup\left\{P^{1}\right\}$. Assume that $g\left(P^{1}\right)=0$ and that $g$ maps the portion of $c$ inside $N_{0}$ isometrically into the positive real axis in the punctured disc. Then $g=f$ (as germs). Any analytic continuation of $f$ is hence called a horocyclic coordinate at $P^{1}$ relative to $P^{2}$.

Proof. Let $\varphi=g \circ f^{-1}$. Then $\varphi$ is defined in some neighborhood $N_{1}$ of the origin, $\varphi$ has a simple zero at $0, \varphi\left(I \cap N_{1}\right) \subset I$ and for some fixed $\varepsilon>0$ we have

$$
\log \left(\frac{\log \varphi\left(x_{1}\right)}{\log \varphi\left(x_{2}\right)}\right)=\log \frac{\log x_{1}}{\log x_{2}} \text { for all } 0<x_{2}<x_{1}<\varepsilon .
$$

We conclude that $\log \varphi(x) / \log x$ is a constant $\alpha$ for $x \in I \cap N_{1}$. Hence $\log \varphi(z)=\alpha \log z$, all $z \in N_{1}-\{0\}$. Choose the branch of $\log \varphi$ which is real on $I \cap N_{1}$ and continue it analytically around the origin. Since $\varphi(z)=z \psi(z)$, for $|z|$ small, with $\psi$ holomorphic and nonzero at 0 , it follows that the continuation 
of $\log \varphi$ around the origin leads to $\log \varphi+2 \pi i$. Thus $\alpha=1$ and $\varphi$ is the identity map.

Remarks. For the horocyclic coordinate $f$ with domain $D$ defined by (1.5.2), we have:

(1) The image $f(D)$ contains the disc of radius $e^{-\pi / 2}$ about the origin.

(2) For all $\theta \in \mathbb{R}$, the curve $\left\{z \in D-\left\{P^{1}\right\} ; \arg f(z)=\theta\right\}$ is a geodesic on the surface $S$.

(3) For $0<r_{1}<r_{2}<e^{-\pi / 2}$, the two parallel circles (which we will call horocircles on $S$ relative to the puncture $\left.P^{1}\right)\left\{z \in D-\left\{P^{1}\right\} ;|f(z)|=r_{j}\right\}, j=$ 1,2 , are at distance $\frac{1}{\pi} \log \left(r_{2} / r_{1}\right)$ from each other. The interior of a horocircle is called a horodisc (it is, of course, a punctured disc on $S$ ).

(4) The domain $D$ contains the geodesic $c\left(P^{1}, P^{3}\right)=\tilde{c}$ and $\lim _{s \rightarrow-\infty} f(\tilde{c}(s))$ $=-1$.

(5) The domain of a horocyclic coordinate is not well defined. The germ of any horocyclic coordinate at a puncture relative to another is well defined. Our constructions involving horocyclic coordinates will depend only on the germ of the coordinate (by uniqueness of analytic continuation). Hence it makes sense to speak of "the" horocyclic coordinate at $P^{1}$ relative to $P^{2}$.

Corollary. (a) If $f_{1}$ is the horocyclic coordinate at $P^{1}$ relative to $P^{3}$ then $f_{1}=$ $-f$.

(b) If $f_{2}$ is the horocyclic coordinate at $P^{2}$ relative to $P^{1}$ then $(\log f)\left(\log f_{2}\right)$ $=\pi^{2}$ (use, in each case, the branch of the logarithm that is real on $c$ ).

1.6. We leave it to the reader to verify the following

Proposition. (a) Horodiscs of radii less than $e^{-\pi}$ about distinct punctures on $S$ are disjoint. Any two horodiscs of radii $r, e^{-\pi}<r<e^{-\pi / 2}$ (about distinct punctures), intersect nontrivially.

(b) Horodiscs of radii $r, e^{-\sqrt{3} \pi / 2}<r<e^{-\pi / 2}$, about the three punctures cover all of $S$ (but no smaller number will do).

Remarks. (1) For each $r$ with $0<r \leq e^{-\pi / 2}$, we can look at the space $Y_{r}$ obtained by deleting from $S$ the open horodiscs of radii $r$ about each of the punctures. For $0<r<e^{-\pi}, Y_{r}$ is a pair of pants; that is, $Y_{r}$ is a bordered Riemann surface whose interior is topologically equivalent to $S$. For $r=e^{-\pi}$, each pair of boundary curves of $Y_{r}$ intersects in a single point: "the midpoint" of the (infinite length) simple geodesic connecting the corresponding punctures.

(2) Let $z_{j}$ be a horocyclic coordinate at $P^{j}, j=1,2$. Let us consider horocircles of radii $r_{j}$ (in the coordinate $z_{j}, 0<r_{1}<e^{-\pi / 2}$ ). The product $\left(\log r_{1}\right)\left(\log r_{2}\right)$ determines the relative position of the two horocircles: they are disjoint if the product is greater than $\pi^{2}$, they are tangent at one point if the product equals $\pi^{2}$ and they intersect in two points if the product is less than $\pi^{2}$. This observation will be useful in $\S 2.1$. 
1.7. The modular group $\operatorname{PSL}(2, \mathbb{Z})$ is the normalizer of $F$ in $\operatorname{PSL}(2, \mathbb{R})$. Note that $\operatorname{PSL}(2, \mathbb{Z}) / F \cong \operatorname{Aut}\left(\mathbb{H}^{2} / F\right) \cong \mathscr{S}_{3}=$ permutation group on three letters. The two Möbius transformations

$$
A^{1 / 2}=\left[\begin{array}{ll}
1 & 1 \\
0 & 1
\end{array}\right], \quad B^{1 / 2}=\left[\begin{array}{cc}
1 & 0 \\
-1 & 1
\end{array}\right],
$$

for example, generate $\operatorname{PSL}(2, \mathbb{Z}) / F$. For each puncture $P$ on $S$, there exists an automorphism $\varphi$ of $S$ which fixes $P$ and interchanges the other two punctures. If $z$ is a horocyclic coordinate at the puncture $P$, then the automorphism $\varphi$ is completely described by $\varphi(z)=-z$.

The normalizer $N(F)$ of $F$ in $\operatorname{PSL}(2, \mathbb{C})$ is the $\mathbb{Z}_{2}$-extension of $\operatorname{PSL}(2, \mathbb{Z})$ by the motion $E_{0}=i\left[\begin{array}{cc}1 & 0 \\ 0 & -1\end{array}\right]$. This normalizer may be identified with $\operatorname{PGL}(2, \mathbb{Z})$. Further, $\operatorname{PGL}(2, \mathbb{Z}) / F \cong \mathscr{S}_{3} \oplus \mathbb{Z}_{2}$. It follows that if $E \in N(F)$ satisfies $E \circ A \circ E^{-1}=A^{ \pm 1}$, then there are only two possibilities: either

(I) $E\left(\mathbb{H}^{2}\right)=\mathbb{H}^{2}$ and $E=A^{k / 2}$ with $k \in \mathbb{Z}$ (in this case $E \circ A \circ E^{-1}=A$ ), or

(II) $E\left(\mathbb{H}^{2}\right)=\mathbb{H}_{*}^{2} \quad(=$ the lower half-plane $)$ and $E=E_{0} \circ A^{k / 2}$ with $k \in \mathbb{Z}$ (in this case $E \circ A \circ E^{-1}=A^{-1}$ ).

\section{The $z w=t$ Plumbing CONSTRUCtion}

This section describes the basic plumbing construction. We build surfaces of type $(0,4)$ by plumbing two surfaces of type $(0,3)$. Surfaces of type $(1,1)$ are constructed by plumbing a single $(0,3)$ surface. The limiting cases of these constructions lead to surfaces with nodes.

2.1. Let $S$ be the thrice punctured sphere and let $z$ be a horocyclic coordinate at a puncture on $S$. For $0<r<e^{-\pi / 2}$, let

$$
S_{(r)}=S-\{P \in S ; 0<|z(P)| \leq r\} .
$$

Define $S_{(0)}=S$. If $w$ is a horocyclic coordinate at another puncture of $S$, then

$$
\left\{Q \in S ; 0<|w(Q)|<r^{*}\right\} \subset S_{(r)},
$$

for $r^{*}<\min \left\{e^{-\pi / 2}, e^{\pi^{2} / \log r}\right\}$. See $\S 1.6$.

2.2. Consider two thrice punctured spheres $S^{1}$ and $S^{2}$. Let $z$ and $w$ be horocyclic coordinates at punctures $P^{1}$ and $P^{2}$ of $S^{1}$ and $S^{2}$, respectively. For each $t \in \mathbb{C}$ with $0<|t|<e^{-\pi}$, we construct a Riemann surface $S_{t}$ by introducing an equivalence relation on the disjoint union $S_{(r)}^{1} \cup S_{(r)}^{2}$, where $r=e^{\pi / 2}|t|$. A point $P \in S_{(r)}^{1}$ is identified with a point $Q$ on $S_{(r)}^{2}$ if and only if

$$
z(P) w(Q)=t .
$$

See Figure 4 on page 515 . Clearly $S_{t}$ is a Riemann surface of type $(0,4)$. There exist canonical embeddings $S_{(r)}^{j} \rightarrow S_{t}, j=1,2$. The surface $S_{t}$ contains the 
annulus $\mathscr{A}_{t}$; it is described in terms of the $z$ and $w$ coordinates by

$$
\left\{P \in S_{t} ; r<|z(P)|<e^{-\pi / 2}\right\}=\left\{Q \in S_{t} ; r<|w(Q)|<e^{-\pi / 2}\right\} .
$$

The modulus of $\mathscr{A}_{t}$ is $-\frac{1}{2}-\frac{1}{2 \pi} \log |t|$. The complement of the closure of $\mathscr{A}_{t}$ in $S_{t}$ is the disjoint union $S_{\left(e^{-\pi / 2}\right)}^{1} \cup S_{\left(e^{-\pi / 2}\right)}^{2}$. Each puncture on $S_{t}$ still has a horocyclic coordinate; however, its domain of definition has changed. The image of such a coordinate may no longer cover, for example, the disc of radius $e^{-\pi / 2}$ about the origin. If, however, $|t|<e^{-3 \pi / 2}$, then $\left(r<e^{-\pi}\right.$ and $)$ each of the horocyclic mappings on $S_{t}$ covers the disc of radius $e^{-\pi}$ about zero. See Remark (2) of $\S 1.6$.

By construction, the surface $S_{t}$ has a distinguished simple closed loop in $\mathscr{A}_{t}$. It is described in terms of the $z$ and $w$ coordinates by

$$
\left\{P \in S_{t} ;|z(P)|=\sqrt{|t|}\right\}=\left\{Q \in S_{t} ;|w(Q)|=\sqrt{|t|}\right\} \text {. }
$$

This curve will be called the central curve on $\mathscr{A}_{t}$; it partitions $S_{t}$ into two (topological) thrice punctured spheres (see also §2.4).

Remark. The horocyclic coordinates on $S_{t}$ are defined by the embeddings of $S_{(r)}^{j}, j=1$ and 2 , into $S_{t}$. If one represents the surface $S_{t}$ as $\Delta / \Gamma$ with $\Gamma$ a Kleinian group with invariant component $\Delta$, then the parabolic elements of $\Gamma$ define horocycles on $S_{t}$. These horocycles do not in general agree with the horocycles determined by our horocyclic coordinates. They will agree if $\Gamma$ is a terminal $b$-group with the accidental parabolic elements of $\Gamma$ corresponding to the central curves on $S_{t}$ (see $\left.\S 7.2\right)$.

2.3. Several choices were made in the above construction. For a given $t \in \mathbb{C}$, $0<|t|<e^{-\pi}$, we chose $r=e^{\pi / 2}|t|$. We can choose any $r \in \mathbb{R}$ with $e^{\pi / 2}|t| \leq$ $r<\sqrt{|t|}$. The construction leads to the same surface, since in each case $z$ is being continued analytically by defining $z=t / w$ on part of the domain of $w$. To construct families of surfaces depending analytically on $t$, it is convenient to use values of $r$ that depend on the range of the $t$ parameter.

Special case. Consider the case $0<|t|<e^{-2 \pi}$ and $r=e^{\pi}|t|<e^{-\pi}$. The plumbing construction takes place in the annuli

$$
\left\{P \in S^{1} ; r<|z(P)|<e^{-\pi}\right\} \text { and }\left\{Q \in S^{2} ; r<|w(Q)|<e^{-\pi}\right\} .
$$

Thus the open horodiscs of radii $e^{-\pi}$ about each of the four punctures on $S_{t}$ are disjoint (and we can repeat our construction).

The plumbing construction. In the above construction we have used annuli that are filled out by horocircles. We shall say that the plumbing construction is tame. We proceed to describe the not necessarily tame plumbing construction. Let $a_{1}$ and $a_{2}$ be simple closed curves on $S^{1}$ and $S^{2}$ which are contractible to the punctures $P^{1}$ and $P^{2}$, respectively. Let $\mathscr{A}_{i}(i=1,2)$ be an annular neighborhood of $a_{i}$. Assume that $\mathscr{A}_{1}$ (respectively, $\mathscr{A}_{2}$ ) is contained in a 


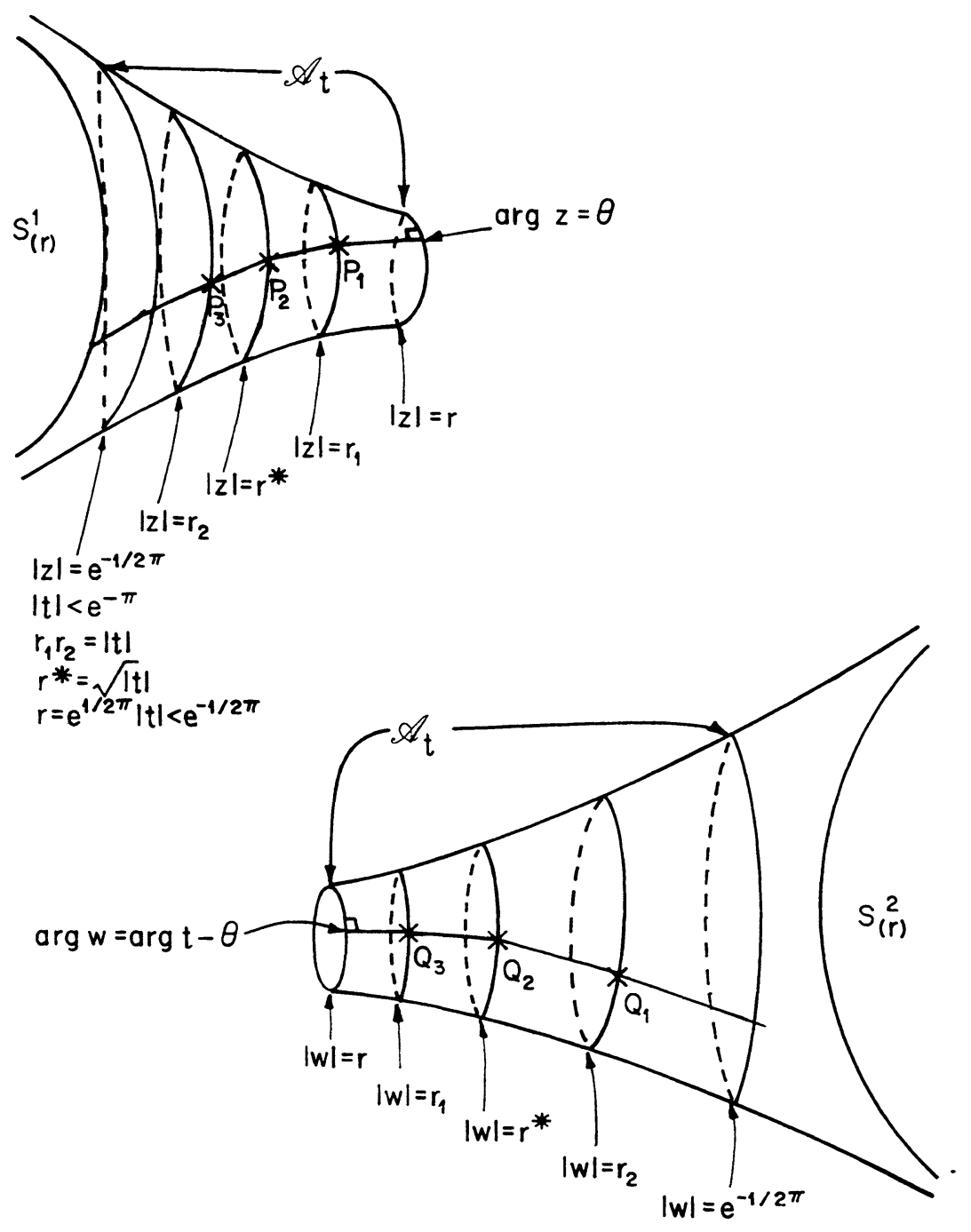

FIGURE 4. The plumbing construction on disjoint surfaces.

domain for a horocyclic coordinate $z \quad(w)$ at $P^{1} \quad\left(P^{2}\right)$. The complement of $\mathscr{A}_{i}$ in $S^{i}$ consists of two components; precisely one of these is a punctured disc. Let

$$
S_{\text {truncated }}^{i}=S^{i}-\{\text { closure of this punctured disc }\} .
$$

The annulus $\mathscr{A}_{i}$ has two boundary components; precisely one of these, the inner boundary, is part of boundary $S_{\text {truncated }}^{i}$ (we call the other one the outer boundary of $\mathscr{A}_{i}$ ). Assume now that there exists a one-to-one holomorphic map 
$f$ of $\mathscr{A}_{1}$ onto $\mathscr{A}_{2}$ with

$f\left(\right.$ outer boundary of $\left.\mathscr{A}_{1}\right)=$ inner boundary of $\mathscr{A}_{2}$,

and there exists a $t \in \mathbb{C}^{*}$, such that the map $f$ is given by $w=f(z)=t / z$. We define a surface $S$ by introducing an equivalence relation on the disjoint union $S_{\text {truncated }}^{1} \cup S_{\text {truncated }}^{2}$; a point $P \in \mathscr{A}_{1}$ is identified with its image $f(P) \in \mathscr{A}_{2}$. In this case, we shall say that the surface $S=S_{t}$ has been obtained from $S^{1}$ and $S^{2}$ by the (not necessarily tame) plumbing construction or operation with gluing or plumbing parameter $t$. It is clear that $S_{t}$ carries (as before) a central curve (namely, $a_{1}$ ). The important feature of the above definition is that we use distinguished coordinates to define the gluing parameters. The surface $S_{t}$ has been constructed by introducing an equivalence relation: a point $P$ on $\mathscr{A}_{1}$ is identified with a point $Q$ on $\mathscr{A}_{2}$ if and only if $z(P) w(Q)=t$. This relation can be used to extend the definition of the $z$ and $w$ coordinates to an open set containing the image of $\mathscr{A}_{1} \cup \mathscr{A}_{2}$ in $S_{t}$. It is not obvious that the surface $S_{t}$ depends only on $t$ (and not on the choice of the two annuli). We will show, using Kleinian groups, that the gluing construction is independent of the choice of annuli. See Theorems 6.2 and 7.3. For an alternate direct proof see $\S 14$. From now on, unless otherwise indicated, "plumbing" means "not necessarily tame plumbing".

Remarks. (1) The Klein four group acts as a group of automorphisms on $S_{t}$. Using the $z$ and $w$ coordinates (the relation $z w=t$ can be used to extend $z$ and $w$ to be defined on the complement in $S_{t}$ of two simple curves; each of these curves joins two punctures), we describe the three involutions:

$$
\begin{gathered}
J_{1}(P)=Q \Leftrightarrow z(P)=-z(Q), \\
J_{2}(P)=Q \Leftrightarrow z(P)=w(Q), \\
J_{3}=J_{2} \circ J_{1}=J_{1} \circ J_{2} .
\end{gathered}
$$

(2) Note that (see also §6.2) $S_{t}$ is conformally equivalent to $S_{-t}$ (they have, however, different "markings"); and thus the conformal equivalence class of $S_{t}$ does not depend on the choice of horocyclic coordinate (we can replace $z$ by $-z$ and/or $w$ by $-w)$.

(3) We will show that every marked surface can be constructed by a finite number of plumbings. We will have to use nontame plumbings. It is not known whether every surface (ignoring markings) can be constructed using only tame constructions with horocyclic coordinates. See $\S \S 6.1,6.3$, and 7.5.

2.4. We modify the tame constructions of $\S \S 2.2$ and 2.3 to the case of a single sphere. Let $z$ and $w$ be horocyclic coordinates at distinct punctures of the thrice punctured sphere $S$. Choose $t \in \mathbb{C}$ with $0<|t|<e^{-2 \pi}$. We consider (here $r=e^{\pi}|t|$ )

$$
S_{(r)}^{*}=S-(\{P \in S ; 0<|z(P)| \leq r\} \cup\{Q \in S ; 0<|w(Q)| \leq r\}) .
$$


The equivalence relation on $S_{(r)}^{*}$ identifies two points $P$ and $Q$ if and only if they satisfy $z(P) w(Q)=t$. The resulting Riemann surface $S_{t}$ is of type $(1,1)$. See Figure 5 on page 518 . The surface $S_{t}$ contains the annulus $\mathscr{A}_{t}$ described in terms of the $z$ and $w$ coordinates by

$$
\left\{P \in S_{t} ; r<|z(P)|<e^{-\pi}\right\}=\left\{Q \in S_{t} ; r<|w(Q)|<e^{-\pi}\right\},
$$

respectively. The modulus of $\mathscr{A}_{t}$ is $-1-\frac{1}{2 \pi} \log |t|$. The complement of the closure of $\mathscr{A}_{t}$ in $S_{t}$ is $S_{\left(e^{-\pi}\right)}^{*}$. The surface $S_{t}$ also contains two intersecting

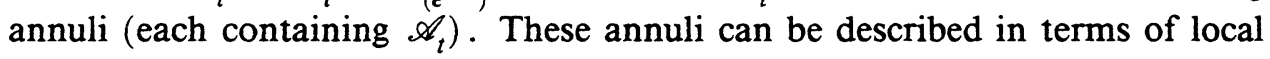
coordinates by

$$
\left\{P \in S_{t} ; r<|z(P)|<e^{-\pi / 2}\right\}, \quad\left\{Q \in S_{t} ; r<|w(Q)|<e^{-\pi / 2}\right\},
$$

respectively. Each of these annuli has modulus $-\frac{3}{4}-\frac{1}{2 \pi} \log |t|$. The puncture on $S_{t}$ has a horocyclic coordinate whose image always contains the disc of radius $e^{-\pi}$. It contains the disc of radius $e^{-\pi / 2}$ whenever $|t|<e^{-3 \pi}$ (that is, whenever $\left.r<e^{-2 \pi}\right)$. Each of the surfaces $S-\{P \in S ; 0<|z(P)| \leq r\}$ and $S-\{Q \in S ; 0<|w(Q)| \leq r\}$ embeds into $S_{t}$. The central curve described by (2.2.2) provides a maximal partition of $S_{t}$.

Remarks. (1) The surface $S_{t}$ has an involution $J$ acting on it. It is described in terms of the $z, w$ coordinates by formula (2.3.3) for $J_{2}$.

(2) Let $P^{1}, P^{2}, P^{3}$ be the three punctures on $S$. Let $f_{i j}$ be the horocyclic coordinate at $P^{i}$ relative to $P^{j}$. Let $S_{t}$ be the surface obtained for $z=f_{31}$ and $w=f_{13}$ and gluing parameter $t$. Similarly, let $\widetilde{S}_{t}$ be the surface for $z=f_{32}$ and $w=f_{13}$ and gluing parameter $t$. Then $\widetilde{S}_{t}$ is conformally equivalent to $S_{-t}$ (as surfaces with a partition). However, in general, $S_{t}$ is not conformally equivalent to $S_{-t}$ (see $\left.\S 11.8\right)$.

2.5. We have seen that for $0<|t|<e^{-2 \pi}$, the constructions described above are tame, involve two horodiscs of radii $e^{-\pi}$ and do not affect the remaining horodiscs of radii $<e^{-\pi}$. This permits us to iterate the constructions. The combinatorial machinery for the general construction will be described in $\S 3$. It is obvious that in the constructions of $\S \S 2.2$ and 2.3 , the thrice punctured spheres may be replaced, by induction, by arbitrary surfaces with horocyclic coordinates defined at the punctures. Similarly, the construction of $\S 2.4$ involving a single Riemann surface and its nontame generalization can be carried out on an arbitrary hyperbolic surface with two or more punctures (with horocyclic coordinates defined at the punctures).

2.6. The above constructions can be extended to $t=0$. Consider the fiber space with total space

$$
\mathbf{V}=\left\{(z, w, t) \in \mathbb{C}^{3} ;|t|<e^{-2 \pi},|z|<e^{-\pi},|w|<e^{-\pi}, z w=t\right\}
$$

base space

$$
\mathbf{B}=\left\{t \in \mathbb{C} ;|t|<e^{-2 \pi}\right\},
$$




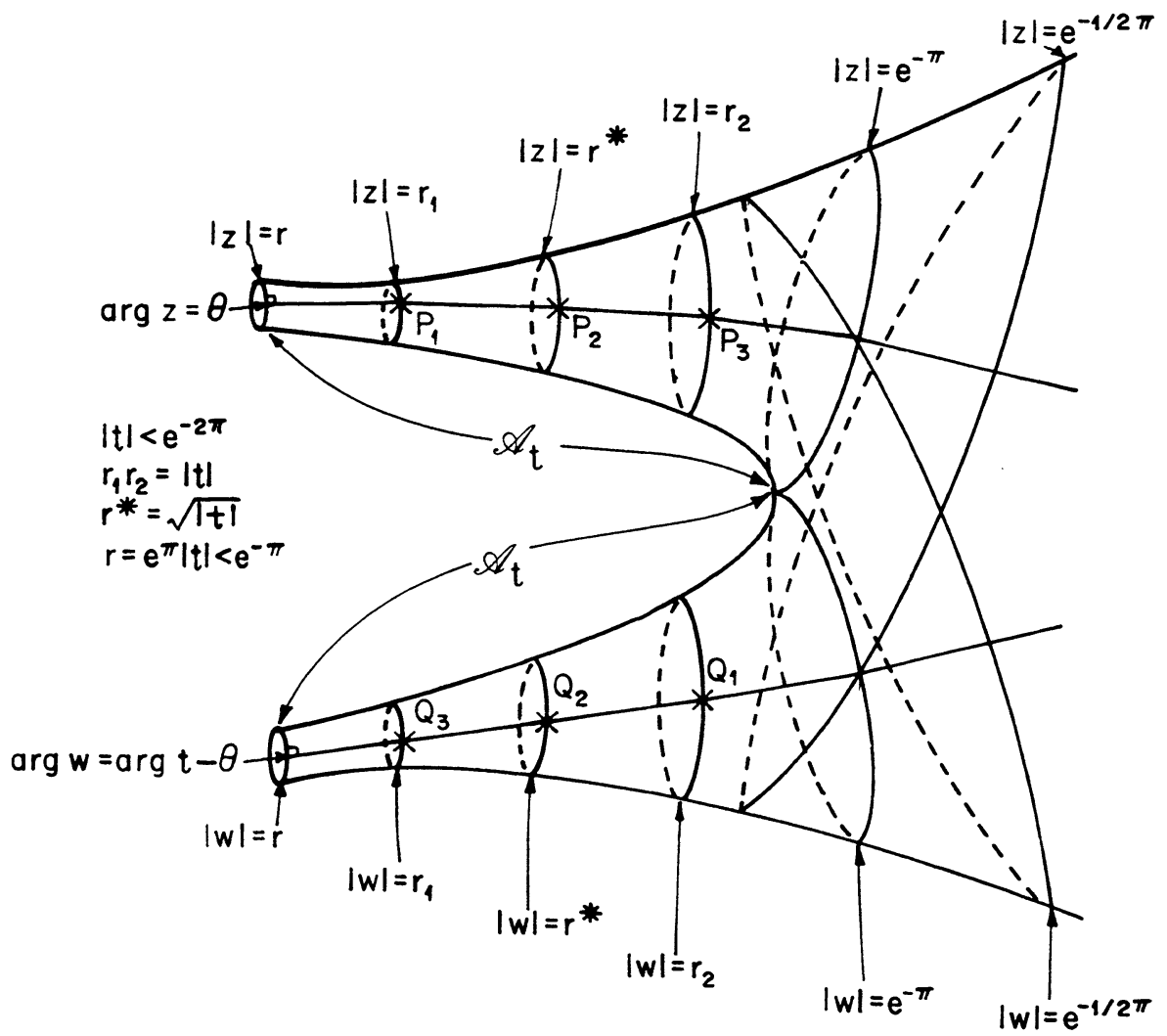

FIGURE 5. The plumbing construction on a single surface.

and projection $\rho$ that sends $(z, w, t)$ to $t$. Note that $\mathbf{V}$ is a complex manifold of dimension 2 (the Jacobian matrix of $z w=t$ has rank 1 everywhere). The fiber $\rho^{-1}(t)$ over $t \neq 0$ is the annulus $\mathscr{A}_{t}$ described in $z$ and $w$ coordinates by

$$
\left\{z \in \mathbb{C} ; e^{\pi}|t|<|z|<e^{-\pi}\right\}=\left\{w \in \mathbb{C} ; e^{\pi}|t|<|w|<e^{-\pi}\right\} ;
$$

the relation between the $z$ and $w$ coordinates on $\mathscr{A}_{t}$ is, of course, $z w=t$. The fiber over 0 is the singular Riemann surface $\left\{(z, w) \in \mathbb{C}^{2} ;|z|<e^{-\pi}\right.$, $\left.|w|<e^{-\pi}, z w=0\right\}$. It has a node at $(0,0)$ and is nonsingular elsewhere. We proceed to enlarge the fiber space (same base) so that the fiber over a point $t \neq 0$ is $S_{t}$ (as defined in $\S \S 2.2$ and 2.4).

For the $(0,4)$ construction, consider the fiber spaces $\mathbf{V}_{1}, \mathbf{V}_{2}$ each over $\mathbf{B}$ whose fibers over $t$ are the Riemann surfaces $S_{(\sqrt{|t|})}^{1}$ and $S_{(\sqrt{|t|})}^{2}$ (as defined by (2.1.1) with respect to the local coordinates introduced in $\S 2.2$ which we now label $z_{1}$ and $w_{2}$ ). The fibers over 0 are $S^{1}$ and $S^{2}$ (respectively). A point $(P, t)$ in $\mathbf{V}_{1}$ (thus $\left.P \in S_{(\sqrt{|t|})}^{1}\right)$ is identified with $\left(z, w, t_{1}\right)$ in $\mathbf{V}$ if and only if $t=t_{1}$ and $z_{1}(P)=z$. Similarly, a point $(Q, t)$ in $\mathbf{V}_{2}$ is identified 
with $\left(z, w, t_{1}\right)$ in $\mathbf{V}$ if and only if $t=t_{1}$ and $w_{2}(Q)=w$. The disjoint union $\mathbf{V}_{1} \cup \mathbf{V} \cup \mathbf{V}_{2}$ with the equivalence relation introduced above is a complex manifold $\mathbf{V}(0,4)$. Each of the manifolds $\mathbf{V}, \mathbf{v}_{1}$ and $\mathbf{V}_{2}$ embeds into $\mathbf{V}$. The projection $\mathbf{V}(0,4) \rightarrow \mathbf{B}$ defines a fiber space whose fiber over $t \neq 0$ is $S_{t}$ (of $\S 2.2$ ) and over $t=0$ is a four times punctured sphere with a single node (it is $S^{1} \cup S^{2}$ with the punctures corresponding to the origins of the horocyclic coordinates identified to form a node).

The $(1,1)$ construction is quite similar. We let $\mathbf{V}_{0}$ be the fiber space over B whose fiber at $t \neq 0$ is $S_{(\sqrt{|t|})}^{*}$ (see $\left.\S 2.4\right)$. The fiber over 0 is $S$. The identifications are

$$
\left(z_{1}(P), t\right)=(z, w, t) \text { and }(z, w, t)=\left(w_{2}(Q), t\right)
$$

(using obvious notation conventions). The fiber space $\mathbf{V}(1,1)$ is the disjoint union $\mathbf{V}_{0} \cup \mathbf{V}$ modulo the above equivalence relation. The fiber over $t \neq 0$ is $S_{t}$ (of $\$ 2.4$ ) and the fiber over 0 is the surface $S$ with the origins of two horocyclic coordinates identified to form a node.

The above considerations lead us to define the plumbing constructions for $t=0$. If $S^{1}$ and $S^{2}$ are two Riemann surfaces and $P^{j}$ is a puncture on $S^{j}$ $(j=1,2)$, then $S_{0}$ is the surface obtained by identifying $P^{1} \in S^{1} \cup\left\{P^{1}\right\}$ with $P^{2} \in S^{2} \cup\left\{P^{2}\right\}$ to form a node. Similarly for a single surface with two punctures.

\section{THE PLUMBING CONSTRUCTION FOR AN ADMISSIBLE GRAPH}

In this section we describe how to construct an arbitrary surface of finite hyperbolic type from a collection of thrice punctured spheres by a series of plumbings. An admissible graph controls the topological and combinatorial aspects of the construction and gluing parameters control the analytic aspects. A moduli space is constructed for each admissible graph.

3.1. Let $(p, n)$ be a pair of nonnegative integers with $v=2 p-2+n>0$. Then $d=3 p-3+n \geq 0$. For $t=\left(t_{1}, \ldots, t_{d}\right) \in \mathbb{C}^{d}$, we let $|t|$ be its $L^{\infty}$-norm (see (0.1)). We construct a region $\mathbf{D} \subset \mathbb{C}^{d}$ that covers part of the compactification of $\overline{\mathbf{R}(p, n)}$. The complement in $\mathbf{D}$ of the coordinate hyperplanes is an intermediate covering space lying above $\mathbf{R}(p, n)$ and below $\mathbf{T}(p, n)$. Here $\mathbf{T}(p, n)$ is the Teichmüller space of Riemann surfaces of finite analytic type $(p, n)$, $\mathbf{R}(p, n)$ is the moduli or Riemann space of conformal equivalence classes of surfaces of type $(p, n)$ and $\overline{\mathbf{R}(p, n)}$ is its compactification. For each point $t$ in $\mathbf{D}$, we construct a Riemann surface $S_{t}$. This surface may have nodes and has a partial marking by simple closed partition curves. The complement of the nodes and partition curves is a union of $v$ pairs of pants. In order to organize our data, it is convenient to introduce a class of (almost trivalent) graphs.

3.2. Consider a connected labeled graph $\mathscr{G}$ on $v(\mathscr{G})=v$ vertices $\left(S^{1}, S^{2}, \ldots\right.$, $\left.S^{v}\right)$ and $d(\mathscr{G})=d$ edges $\left(a_{1}, \ldots, a_{d}\right)$. We assume that at most three edges 
meet at any one vertex. A graph $\mathscr{G}$ of the above type will be called an admissible graph of type $(p, n)$. We associate a sphere with three holes to each vertex of $\mathscr{G}$. If two vertices are joined by an edge, then we glue the corresponding spheres along boundary curves. This pair of boundary curves then forms a partition curve. We obtain a (topological) surface $S$ of type $(p, n)$ and a set of $d$ curves $\Sigma$ that partitions $S$ into a union of $v$ pairs of pants. The topological data $(S, \Sigma)$ is uniquely determined by the graph $\mathscr{G}$; we shall call it the surface (with maximal partition) corresponding to the graph. Conversely, every surface $S$ of type $(p, n)$ with a maximal partition $\Sigma$ determines an admissible graph $\mathscr{G}$ of type $(p, n)$.

We augment our graph slightly. If fewer than three, say $i=0,1$, or 2 , edges meet at a vertex, then we adjoin to the graph $3-i$ phantom edges emanating from this given vertex. A phantom edge does not connect the vertex either to itself or to another vertex. An admissible graph of type $(p, n)$ has exactly $n$ phantom edges (these will be labeled $a_{d+1}, \ldots, a_{d+n}$ ) and the augmented graph (that is, the union of $\mathscr{G}$ and the phantom edges) has precisely three edges or phantom edges emanating from each vertex.

Remark. There is precisely one admissible graph of type $(p, n)$ for $(p, n)=$ $(0,3),(0,4)$ or $(1,1)$. The augmented graph of the type $(0,3)$ consists of one vertex and three phantom edges emanating from it. The graph of type $(0,4)$ consists of two vertices and one edge joining them plus two phantom edges emanating from each vertex. The augmented graph of type $(1,1)$ consists of one vertex, one edge (joining the vertex to itself) and an additional phantom edge. See Figure 6.

Let $S^{j}, j=1, \ldots, v$, be a vertex of $\mathscr{G}$. Each of the three edges or phantom edges emanating from $S^{j}$ is associated with a (topological) puncture on the surface $S^{j}$. We label the punctures using the ordering on the edges emanating from this vertex (if an edge $a_{k}$ joins $S^{j}$ to itself, then we arbitrarily assign an ordering to the two punctures connected by $a_{k}$ ). The three punctures on $S^{j}$ are labeled as $P^{j i}$. We interpret $i$ as an integer $\bmod 3$ corresponding to the cyclic ordering of the punctures.

The edges of $\mathscr{G}$ can be oriented. If the edge $a_{k}$ joins $S^{j}$ to $S^{j^{\prime}}$ with $j<j^{\prime}$, then the positive orientation of $a_{k}$ goes from $S^{j}$ to $S^{j^{\prime}}$ (thus $a_{k}^{-1}$ is the same edge oriented from $S^{j^{\prime}}$ to $S^{j}$ ). If the edge $a_{k}$ joins a vertex to itself, then we arbitrarily assign an orientation to it; in this case $a_{k}$ and $a_{k}^{-1}$ are two of the three edges starting from the vertex. Each phantom edge is oriented to start at a vertex (it ends at a puncture not associated to any vertex). It is also useful to have a second labeling for the punctures $P^{j i}$. An edge $a_{k}$ starts at the puncture (to be labeled) $P_{k}^{\text {initial }}$ and ends at the puncture $P_{k}^{\text {terminal }}$. Phantom edges have only initial punctures in this sense.

It involves no loss of generality to assume that the edges $a_{1}, \ldots, a_{d}$ (excluding the phantom edges) have been ordered so that the graph $\mathscr{G}_{k}$ obtained by 


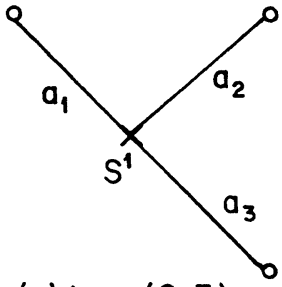

(a) type $(0,3)$

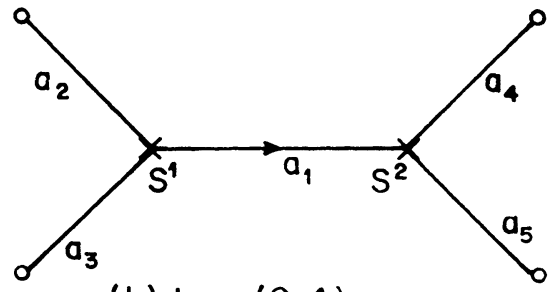

(b) type $(0,4)$

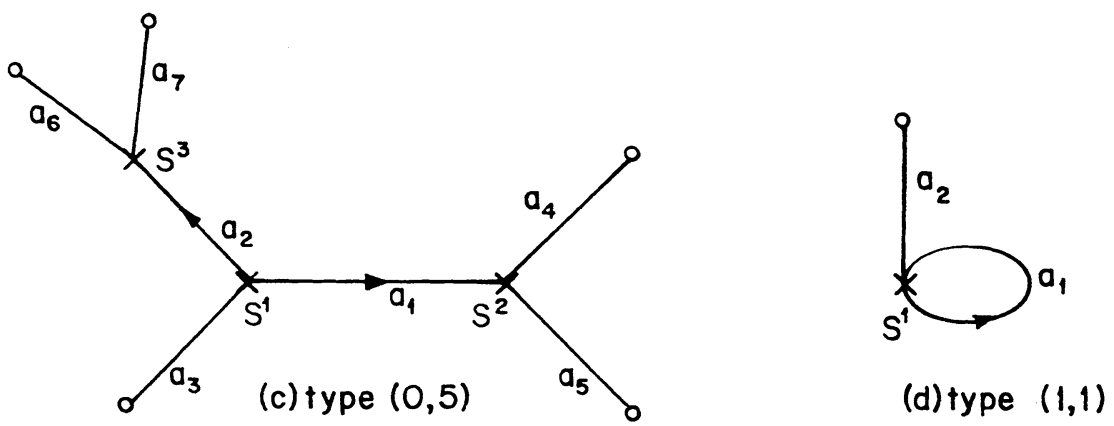

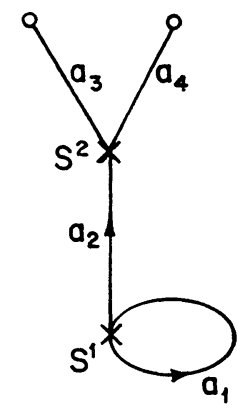

(e) type $(1,2)$

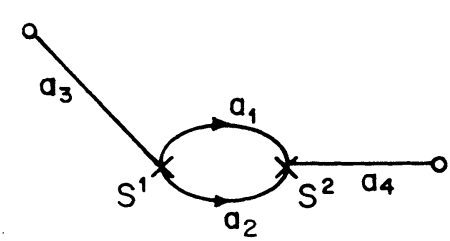

(f) type $(1,2)$

FIGURE 6. The six simplest augmented graphs. Note that the only signatures for which there exists precisely one graph are: $(0,3),(0,4),(0,5)$ and $(1,1)$.

restricting $\mathscr{G}$ to the first $k$ edges $a_{1}, \ldots, a_{k}$, and all the vertices they join, is connected (hence also admissible of some type $\left(p^{\prime}, n^{\prime}\right)$ satisfying $0<v^{\prime}=$ $\left.2 p^{\prime}-2+n^{\prime} \leq v\right)$. Here $k=0, \ldots, d, \mathscr{G}_{0}$ is the trivial graph of type $(0,3)$ and thus $\mathscr{G}_{d}=\mathscr{G}$. We note that for $k=0, \ldots, d-1$, the graph $\mathscr{G}_{k+1}$ is obtained by joining $\mathscr{G}_{k}$ to the graph of type $(0,3)$ along phantom edges or by joining two distinct phantom edges on $\mathscr{G}_{k}$ to form an edge. A semi-canonical ordering for the edges of $\mathscr{G}$ is an ordering of the edges of $\mathscr{G}$ with the property that for $k=0, \ldots, v-1$, the graph $\mathscr{G}_{k}$ is of type $(0, k+3)$; thus for $k=v, \ldots, d$, the graph $\mathscr{G}_{k}$ is of type $(k-v+1,3 v-2 k)$. Further, the edge $a_{1}$ starts at the vertex $S^{1}$, for $k=1, \ldots, v-1$ the edge $a_{k}$ starts at a vertex of $\mathscr{G}_{k-1}$ and ends at the new vertex of $\mathscr{G}_{k}$ (it is assigned the lowest possible 
new vertex label), for $k=v, \ldots, d$ the edge $a_{k}$ joins two vertices of $\mathscr{G}_{k}$ that have already been labeled in $\mathscr{G}_{k-1}$, and for $k=d+1, \ldots, d+n$ the phantom edge $a_{k}$ start at a previously labeled vertex of $\mathscr{G}=\mathscr{G}_{d}$.

Remark. Consider the augmented graph $\mathscr{G}$ embedded in $\mathbb{R}^{3}$. The boundary of a regular neighborhood of the augmented graph $\mathscr{G}$ in $\mathbb{R}^{3}$ is (topologically) the surface $S$ of type $(p, n)$ corresponding to $\mathscr{G}$. The edges and phantom edges of $\mathscr{G}$ determine tubes or annuli on $S$. The central curves on each of the annuli determined by the edges (excluding phantom edges) partition $S$ into a union of pairs of pants. The phantom edges determine the punctures on $S$. This surface $S$ will also, at times, be denoted by the symbol $\mathscr{G}$.

3.3. Let $\mathscr{G}$ be an admissible augmented graph of type $(p, n)$. Let $a$ be an edge on $\mathscr{G}$. We break the edge $a$ and form two phantom edges. If the resulting graph $\mathscr{G}^{\prime}$ is still connected, it is an admissible graph of type $(p-1, n+2)$. If the breaking operation results in two graphs $\mathscr{G}^{\prime}$ and $\mathscr{G}^{\prime \prime}$, then these graphs are admissible of types $\left(p^{\prime}, n^{\prime}\right)$ and $\left(p^{\prime \prime}, n^{\prime \prime}\right)$, respectively, with $p=p^{\prime}+p^{\prime \prime}$, $n=n^{\prime}+n^{\prime \prime}-2$. We shall say that the new graph or graphs have been obtained by a break. Conversely, given a graph $\dot{G}^{\prime}$ with two phantom edges, then these two phantom edges can be joined and given an orientation to form a new graph $\mathscr{G}$. We say that $\mathscr{G}$ is an $H N N$-extension of the graph $\mathscr{G}^{\prime}$. Similarly, given two graphs $\mathscr{G}^{\prime}$ and $\mathscr{G}^{\prime \prime}$ each with a phantom edge, then these phantom edges can be joined to form a new graph $\mathscr{G}$ : the AFP of $\mathscr{G}^{\prime}$ and $\mathscr{G}^{\prime \prime}$. It is obvious that the above operations can be iterated. An arbitrary graph of type $(p, n)$ can be constructed from graphs of type $(0,3)$ by $v-1$ AFP-operations followed by $p \mathrm{HNN}$-operations. Special cases of these operations were discussed in $\S 3.2$ when we defined the semicanonical ordering of the edges of a graph.

3.4. We now introduce the family of Riemann surfaces constructed from thrice punctured spheres using plumbing operations. It will turn out that our family contains all surfaces (see $\S \S 7.2$ and 7.6) of finite analytic type.

Let $\mathscr{G}$ be an admissible augmented graph of type $(p, n)$. Let $(S, \Sigma)$ be the topological surface with maximal partition corresponding to the graph $\mathscr{G}$. An admissible Riemann surface of graph type $\mathscr{G}$ ( $\mathscr{G}$-admissible surface, for short) will be a Riemann surface structure (possibly with nodes) on $S$ together with a distinguished atlas of (complex) coordinates on $S$; the atlas will consist of $n$ horocyclic coordinates, one for each puncture on $S$. The distinguished atlas need not cover all of $S$. We define the $\mathscr{G}$-admissible surfaces by induction on $d(\mathscr{G}) \geq 0$.

If $d(\mathscr{G})=0$ (that is, $\mathscr{G}$ is of type $(0,3)$ ), then $\mathscr{S}(\mathscr{G})$ consists of the thrice punctured sphere together with a choice of a horocyclic coordinate at each puncture. Let us assume that we have defined the family $\mathscr{S}(\mathscr{G})$ for all graphs $\mathscr{G}$ with $d(\mathscr{G})<d, d \in \mathbb{Z}^{+}$. To define $\mathscr{S}(\mathscr{G})$ for a graph $\mathscr{G}$ with $d(\mathscr{G})=d$, we break the graph $\mathscr{G}$ at an edge and hence we may assume that $\mathscr{G}$ is either the AFP of $\mathscr{G}^{\prime}$ and $\mathscr{G}^{\prime \prime}$ (then $d\left(\mathscr{G}^{\prime}\right)+d\left(\mathscr{G}^{\prime \prime}\right)=d-1$ ) or $\mathscr{G}$ is an HNN-extension of $\mathscr{G}^{\prime}$ (with $d\left(\mathscr{G}^{\prime}\right)=d-1$ ). 
By induction, we have defined the families $\mathscr{S}\left(\mathscr{G}^{\prime}\right)$ (and $\mathscr{S}\left(\mathscr{G}^{\prime \prime}\right)$, in the first case). In the AFP case, there are phantom edges $a^{\prime}$ on $\mathscr{G}^{\prime}$ and $a^{\prime \prime}$ on $\mathscr{G}^{\prime \prime}$ that form an edge on $\mathscr{G}$. Let $S^{1}$ and $S^{2}$ be surfaces in $\mathscr{S}\left(\mathscr{G}^{\prime}\right)$ and $\mathscr{S}\left(\mathscr{G}^{\prime \prime}\right)$, respectively. Let $z(w)$ be the distinguished horocyclic coordinate on $S^{1}\left(S^{2}\right)$ corresponding to the puncture determined by the phantom edge $a^{\prime}\left(a^{\prime \prime}\right)$. A Riemann surface $S$ will belong to $\mathscr{S}(\mathscr{G})$ if we can find surfaces $S^{1}$ and $S^{2}$ as above, so that $S$ is constructed from them by a not necessarily tame plumbing operation (involving some plumbing parameter $t$ ) using the local coordinates $z$ and $w$. The distinguished local coordinates on $S$ are the remaining distinguished coordinates of $S^{1}$ and $S^{2}$. A similar definition yields the family $\mathscr{S}(\mathscr{G})$ when $\mathscr{G}$ is an HNN-extension of $\mathscr{G}^{\prime}$. A surface $S \in \mathscr{S}(\mathscr{G})$ carries not only $n$ distinguished horocyclic coordinates but also $d$ valid plumbing parameters. The plumbing parameters uniquely determine the Riemann surface. We establish this fact in $\S \S 7.2$ and 14.

\subsection{The deformation space $\mathbf{D}(\mathscr{G})$ associated to the augmented admissible graph} $\mathscr{G}$ is the domain of valid parameters $t \in \mathbb{C}^{d}$ for surfaces in $\mathscr{S}(\mathscr{G})$. We describe the specific choices to construct this space. It will turn out (Theorem 7.2) that this space is independent of the choices. For the time being, we use a graph $\mathscr{G}$ with a semicanonical ordering for the edges and the labeling of the edges, vertices and punctures described in $\S 3.2$.

We let $z_{j i}$ be the horocyclic coordinate at $P^{j i}$ relative to $P^{j i+1}$. It is also convenient to label $z_{k}^{\text {initial }}\left(z_{k}^{\text {terminal }}\right)=z_{j i}$, whenever $P^{j i}=P_{k}^{\text {initial }}\left(P_{k}^{\text {terminal }}\right)$.

We define the region $\mathbf{D}(\mathscr{G}) \subset \mathbb{C}^{d}$ as the set of valid plumbing parameters $t=\left(t_{1}, \ldots, t_{d}\right)$ for surfaces $S_{t} \in \mathscr{S}(\mathscr{G})$ using the above decomposition of $\mathscr{G}$ and choices of horocyclic coordinates. To be specific, observe that the graph $\mathscr{G}_{1}$ is either of type $(0,4)$ or $(1,1)$. Assume that $\mathscr{G}_{1}$ is of type $(0,4)$ and see Figure 6. The edge $a_{1}$ joins the puncture $P^{11}$ on $S^{1}$ to the puncture $P^{21}$ on $S^{2}$. Then $t_{1} \in \mathbf{D}\left(\mathscr{G}_{1}\right)$ if and only if $t_{1}$ is a gluing parameter for the plumbing construction described in $\S 2.3$ with $z=z_{11}$ and $w=z_{21}$. Similarly, for $\mathscr{G}_{1}$ of type $(1,1)$. We use, of course, the plumbing construction described in $\S 2.5$.

Let $1 \leq k \leq d-1$ and $t^{0}=\left(t_{1}, \ldots, t_{k}\right) \in \mathbf{D}\left(\mathscr{G}_{k}\right)$. Then the edge $a_{k+1}$ joins a puncture $P^{j i}$ on $S_{t^{0}}$ to either another puncture $P^{j^{\prime} i^{\prime}}$ on $S_{t^{0}}$ or to a new puncture $P^{j^{\prime} i^{\prime}}$ on a thrice punctured sphere $S$ disjoint from $S_{t^{0}}$. In either case, we have the data for a plumbing construction using horocyclic coordinates $z=z_{j i}$ and $w=z_{j^{\prime} i^{\prime}}$. Now $t=\left(t_{1}, \ldots, t_{k}, t_{k+1}\right) \in \mathbf{D}\left(\mathscr{G}_{k+1}\right)$ if and only if $t_{k+1}$ is a gluing parameter for a plumbing construction of the type described in either $\S 2.3$ or $\S 2.5$, as appropriate, starting with the surfaces $S_{t^{\circ}}$ (and $S$, if necessary).

Theorem. Let $\mathscr{G}$ be an admissible graph of type $(p, n)$. Then

(a) $\left\{t \in \mathbb{C}^{d} ;|t|<e^{-\pi / 2}\right\} \supset \mathbf{D}(\mathscr{G}) \supset\left\{t \in \mathbb{C}^{d} ;|t|<e^{-2 \pi}\right\}$. 
(b) Each point $t \in \mathbf{D}(\mathscr{G})$ represents a Riemann surface (perhaps with nodes) of type $(p, n)$; each edge $a_{k} \in \mathscr{G}$ represents a simple closed curve on $S_{t}$ (if $t_{k} \neq 0$ ) or a node (if $t_{k}=0$ ). These $d$ curves and nodes partition $S_{t}$ into a union of $v$ thrice punctured spheres.

(c) The surfaces $S_{t}$ with $|t|<e^{-2 \pi}$ are constructed using only tame plumbings.

Proof. Only the inclusions in (a) and (c) need verification. The second inclusion in (a) and part (c) follow from the fact that for $|t|<e^{-2 \pi}$ each of the $d$ plumbing constructions takes place in annuli of form (2.3.1) and, by Proposition 1.6(a), all these annuli are disjoint. The first inclusion in (a) follows from $\S \S 6.1$ and 6.3.

Definition. We call $\mathbf{D}(\mathscr{G})$ the Teichmüller or deformation space corresponding to the graph $\mathscr{G}$. Further properties of this space will be obtained in $\S \S 9.4$ and 9.8 .

\subsection{We define}

$\mathbf{D}_{\text {tame }}(\mathscr{G})=\left\{t \in \mathbf{D}(\mathscr{G}) ; S_{t}\right.$ is constructed using only tame plumbings $\}$.

If $\mathscr{G}$ is of type $(0,4)$, then $\mathbf{D}_{\text {tame }}(\mathscr{G})=\Delta_{e^{-\pi}}$, while for $\mathscr{G}$ of type $(1,1)$, $\mathbf{D}_{\text {tame }}(\mathscr{G})=\Delta_{e^{-2 \pi}}$.

Assume $\mathscr{G}$ is a graph of type $(p, n)$ with $n>0$. Let $t \in \mathbf{D}(\mathscr{G})$ and let $P^{1}, \ldots, P^{n}$ be the punctures on $S_{t}$. Let $z_{j}$ be a horocyclic coordinate vanishing at $P^{j}$. Set

$$
r_{j}(t)=\sup \left\{r ; \text { the image of } z_{j} \text { contains the disc of radius } r\right\},
$$

and

$$
r^{*}(t)=\min \left\{r_{1}(t), \ldots, r_{n}(t)\right\} .
$$

Then $r^{*}(t)>0$ for all $t \in \mathbf{D}(\mathscr{G})$. If $|t|<e^{-2 \pi}$, then $t \in \mathbf{D}_{\text {tame }}(\mathscr{G})$ and $r^{*}(t) \geq e^{-\pi}$. For applications, it is of interest to determine lower bounds of $r^{*}(t)$ for arbitrary $t \in \mathbf{D}(\mathscr{G})$. Part of the domain of one of the coordinates $z_{j}$ may have been removed while plumbing at another pair of punctures (different from $\left.P^{1}, \ldots, P^{n}\right)$. This issue complicates the evaluation of $r^{*}(t)$.

3.7. In order to identify the natural automorphisms of $\mathbf{D}(\mathscr{G})$, we develop a combinatorial model for a covering of one noded surface by another. Let $\mathscr{G}$ and $\mathscr{G}^{\prime}$ be two admissible (augmented) graphs. A morphism $\sigma: \mathscr{G} \rightarrow \mathscr{G}^{\prime}$ is a continuous mapping of $\mathscr{G}$ into $\mathscr{G}^{\prime}$ which sends vertices to vertices, edges injectively to edges, phantom edges to phantom edges, and is a local homeomorphism at each vertex. A morphism $\sigma$ induces a map $\sigma_{1}$ from the vertices of $\mathscr{G}$ to those of $\mathscr{G}^{\prime}$ and a second map $\sigma_{2}$ from the edges of $\mathscr{G}$ to those of $\mathscr{G}^{\prime}$. Using obvious notational conventions (see $\S 3.2$ ), we have

$$
\begin{gathered}
\sigma\left(S^{k}\right)=\left(S^{\prime}\right)^{\sigma_{1}(k)}, \quad k=1, \ldots, v, \\
\sigma\left(a_{j}\right)=\left(a^{\prime}\right)_{\sigma_{2}(j)}^{\delta_{j}}, \quad j=1, \ldots, d+n,
\end{gathered}
$$


where $\delta_{j}= \pm 1$. We say that two morphisms are equivalent if they induce the same map on the (oriented) edges (including the plantom edges). Equivalent maps induce the same maps on the vertices. Two morphisms that induce the same map on the vertices need not be equivalent. Note that for the morphism $\sigma$, we have

$$
\begin{gathered}
1 \leq \sigma_{2}(j) \leq d^{\prime}, \quad \text { for } 1 \leq j \leq d, \\
d^{\prime}+1 \leq \sigma_{2}(j) \leq d^{\prime}+n^{\prime}, \quad \text { for } d+1 \leq j \leq d+n, \\
\delta_{j}=1, \text { for } j=d+1, \ldots, d+n
\end{gathered}
$$

We need one more invariant of a morphism. Let $a_{j_{1}}, a_{j_{2}}$ and $a_{j_{3}}$ be the edges that emanate from $S^{k}$ (it could be that an edge and its inverse appear in the list). Assume that these three edges have been listed according to the cyclic ordering they determine for the punctures on $S^{k}$ (this means that $j_{1}<j_{2}<$ $j_{3}$ whenever none of these edges join $S^{k}$ to itself). Then $\sigma\left(a_{j_{1}}\right), \sigma\left(a_{j_{2}}\right)$ and $\sigma\left(a_{j_{3}}\right)$ determine a new cyclic ordering for the punctures on $\sigma\left(S^{k}\right)=\left(S^{\prime}\right)^{\sigma_{1}(k)}$. Let $\eta_{k}=+1$ whenever this new ordering agrees with the cyclic ordering for punctures on $\left(S^{\prime}\right)^{\sigma_{1}(k)}$; otherwise set $\eta_{k}=-1$.

3.8. An isomorphism of graphs is a morphism that is both injecture and surjective; an automorphism of a graph is an isomorphism of the graph onto itself.

Theorem. If $\mathscr{G}$ and $\mathscr{G}^{\prime}$ are isomorphic augmented admissible graphs, then there exists a linear automorphism $A$ of $\mathbb{C}^{d}$ such that $A(\mathbf{D}(\mathscr{G}))=\mathbf{D}\left(\mathscr{G}^{\prime}\right)$.

Proof. Let $\sigma$ be an isomorphism of $\mathscr{G}$ onto $\mathscr{G}^{\prime}$. It follows that both $\mathscr{G}$ and $\mathscr{G}^{\prime}$ are of the same type $(p, n)$. As above, let $\sigma_{1}$ and $\sigma_{2}$ be the maps induced by $\sigma$ on the vertices and edges of the graphs. (We view $\sigma_{1}$ and $\sigma_{2}$ as permutations on $v$ and $d$ letters, respectively.) Associated to $\sigma$ is a complex linear isomorphism $A=\sigma^{*}: \mathbb{C}^{d} \rightarrow \mathbb{C}^{d}$. For $t=\left(t_{1}, \ldots, t_{d}\right) \in \mathbb{C}^{d}$, define

$$
t^{*}=\sigma^{*}(t)=\sigma^{*}\left(t_{1}, \ldots, t_{d}\right)=\left(\varepsilon_{1} t_{\sigma_{2}^{-1}(1)}, \ldots, \varepsilon_{d} t_{\sigma_{2}^{-1}(d)}\right),
$$

where $\varepsilon_{j}= \pm 1$ is defined as follows. The edge $a_{j}$ starts at a vertex $S^{k}$ and ends at a vertex $S^{k^{\prime}}$. We set $\varepsilon_{j}=\eta_{k} \eta_{k^{\prime}}$; the $\eta_{k}$ 's have been defined in $\S 3.7$. Note that $\varepsilon_{j}=+1$ whenever $a_{j}$ joins a vertex to itself.

The map $\sigma^{*}$ sends $\mathbf{D}(\mathscr{G})$ onto $\mathbf{D}\left(\mathscr{G}^{\prime}\right)$. This is a direct consequence of the construction algorithm for Riemann surfaces described in $\S \S 3.4$ and 3.5. We define

$$
\mathbf{D}_{0}(\mathscr{G})=\left\{t=\left(t_{1}, \ldots, t_{d}\right) \in \mathbf{D}(\mathscr{G}) ; t_{j} \neq 0, j=1, \ldots, d\right\} .
$$

It is obvious that $\sigma^{*}\left(\mathbf{D}_{0}(\mathscr{G})\right)=\mathbf{D}_{0}\left(\mathscr{G}^{\prime}\right)$.

Corollary 1. For $t \in \mathbf{D}(\mathscr{G}), S_{\sigma^{*}(t)}$ is conformally equivalent to $S_{t}$. 
Corollary 2. The map $\sigma \mapsto \sigma^{*}$ is a homomorphism from Aut $\mathscr{G}$, the automorphism group of the graph $\mathscr{G}$, to Aut $\mathbf{D}(\mathscr{G})$, the group of complex analytic automorphisms of $\mathbf{D}(\mathscr{G})$.

Remark. The above homomorphism need not be injective. See $\S 9.9$.

3.9. The deformation spaces $\mathbf{D}(\mathscr{G})$ are fibered over lower dimensional spaces. This is the content of the following

Theorem. (a) Assume that $\mathscr{G}$ is an HNN-extension of $\mathscr{G}^{\prime}$. Let $a_{d}$ be the new edge on $\mathscr{G}$. Then the projection $\rho: \mathbb{C}^{d} \rightarrow \mathbb{C}^{d-1}$ defined by $\rho\left(t_{1}, \ldots, t_{d}\right)=$ $\left(t_{1}, \ldots, t_{d-1}\right)$ maps $\mathbf{D}(\mathscr{G})$ (respectively, $\mathbf{D}_{0}(\mathscr{G})$ ) onto $\mathbf{D}\left(\mathscr{G}^{\prime}\right)\left(\mathbf{D}_{0}\left(\mathscr{G}^{\prime}\right)\right)$.

(b) Assume that $\mathscr{G}$ is an AFP of $\mathscr{G}^{\prime}$ and $\mathscr{G}^{\prime \prime}$. Label the edges of $\mathscr{G}^{\prime}$ as $a_{1}, \ldots, a_{d^{\prime}}$, the new edge in $\mathscr{G}$ as $a_{d^{\prime}+1}$ and the edges in $\mathscr{G}^{\prime \prime}$ as $a_{d^{\prime}+2}, \ldots$, $a_{d^{\prime}+1+d^{\prime \prime}}\left(\right.$ note that $\left.d=d^{\prime}+1+d^{\prime \prime}\right)$. Then the projection $\rho: \mathbb{C}^{d} \rightarrow \mathbb{C}^{d^{\prime}} \times \mathbb{C}^{d^{\prime \prime}}$ defined by

$$
\rho\left(t_{1}, \ldots, t_{d}\right)=\left(\left(t_{1}, \ldots, t_{d^{\prime}}\right),\left(t_{d^{\prime}+2}, \ldots, t_{d^{\prime}+1+d^{\prime \prime}}\right)\right)
$$

maps $\mathbf{D}(\mathscr{G})$ (respectively, $\mathbf{D}_{0}(\mathscr{G})$ ) onto $\mathbf{D}\left(\mathscr{G}^{\prime}\right) \times \mathbf{D}\left(\mathscr{G}^{\prime \prime}\right)\left(\mathbf{D}_{0}\left(\mathscr{G}^{\prime}\right) \times \mathbf{D}_{0}\left(\mathscr{G}^{\prime \prime}\right)\right)$.

Remarks. (1) We have not yet shown that $\mathbf{D}(\mathscr{G})$ is a domain in $\mathbb{C}^{d}$. For $t_{0} \in \mathbf{D}(\mathscr{G})$ with $S_{t_{0}}$ not constructed via tame plumbings, it is cumbersome to show that there is a neighborhood of $t_{0}$ in $\mathbb{C}^{d}$ that is contained in $\mathbf{D}(\mathscr{G})$. We will use Kleinian groups to establish this fact as well as the fact that $\mathbf{D}(\mathscr{G})$ is connected. See $\S 9.5$ and Theorem 9.8 .

(2) The above theorem allows us to identify $\mathbf{D}\left(\mathscr{G}^{\prime}\right)$ and $\mathbf{D}\left(\mathscr{G}^{\prime}\right) \times \mathbf{D}\left(\mathscr{G}^{\prime \prime}\right)$ as subspaces of $\mathbf{D}(\mathscr{G})$ in cases (a) and (b), respectively.

(3) Let $\mathscr{G}$ be an admissible graph. A collection of admissible graphs $\mathscr{G}^{\prime}=$ $\left\{\mathscr{G}^{(1)}, \ldots, \mathscr{G}^{(k)}\right\}$ will be called an allowable subgraph of $\mathscr{G}$, if it has been obtained from $\mathscr{G}$ by ( $k$ or more) breaks. It is clear that the theorem generalizes to this setting; that is, an allowable subgraph $\mathscr{G}^{\prime}$ of $\mathscr{G}$ determines a subspace $\mathbf{D}\left(\mathscr{G}^{\prime}\right)$ of $\mathbf{D}(\mathscr{G})$ consisting of those points in $\mathbf{D}(\mathscr{G})$ where a number of coordinates (= the number of breaks) are zero. It is obvious that

$$
\mathbf{D}\left(\mathscr{G}^{\prime}\right)=\mathbf{D}\left(\mathscr{G}^{(1)}\right) \times \cdots \times \mathbf{D}\left(\mathscr{G}^{(k)}\right) .
$$

(4) Let $t^{\prime} \in \mathbf{D}\left(\mathscr{G}^{\prime}\right)$ and $t^{\prime \prime} \in \mathbf{D}\left(\mathscr{G}^{\prime \prime}\right)$. Then the new edge $a_{d^{\prime}+1}$ in $\mathscr{G}$ allows us to define a surface $S_{t}$ from the surfaces $S_{t^{\prime}}$ and $S_{t^{\prime \prime}}$ using the plumbing construction for the edge $a_{d^{\prime}+1}$ by specifying the value of the $\left(d^{\prime}+1\right)$ st coordinate of $t=\left(t^{\prime}, t_{d^{\prime}+1}, t^{\prime \prime}\right)$. The surface $S_{t}$ depends only on $\mathscr{G}$ and $t$ (not the way $\mathscr{G}$ was constructed from its allowable subgraphs). Similarly, for other more general subgraphs of $\mathscr{G}$. See Theorem 7.6.

Problem. For fixed $t^{\prime}$ and $t^{\prime \prime}$ as above, $\rho^{-1}\left(\left(t^{\prime}, t^{\prime \prime}\right)\right)$ is an open subset of $\mathbb{C}$. The shape of this open set is not known. Is it connected? 
3.10. It is convenient to introduce at this point the curve $\mathbf{V}(\mathscr{G})$ over $\mathbf{D}(\mathscr{G})$ :

$$
\mathbf{V}(\mathscr{G})=\bigcup_{t \in \mathbf{D}(\mathscr{G})} S_{t}
$$

with the natural or canonical projection $\pi_{\mathscr{G}}: \mathbf{V}(\mathscr{G}) \rightarrow \mathbf{D}(\mathscr{G})$ defined by $\pi_{\mathscr{G}}\left(S_{t}\right)=$ $t, t \in \mathbf{D}(\mathscr{G})$. The curve $\mathbf{V}(\mathscr{G})$ is a $(d+1)$-dimensional complex manifold. ${ }^{4}$ We let $\mathbf{V}_{0}(\mathscr{G})=\pi_{\mathscr{G}}^{-1}\left(\mathbf{D}_{0}(\mathscr{G})\right)$.

\section{Deformation (Teichmüller) and moduli (RiemanN) SPACES}

This section summarizes the theory of deformations of Kleinian groups, and introduces a class of functions that give coordinates for Teichmüller spaces.

4.1. Let $\Gamma$ be a finitely generated nonelementary Kleinian group. ${ }^{5}$ An isomorphism $\theta: \Gamma \rightarrow \operatorname{PSL}(2, \mathbb{C})$ is geometric if there exists a quasiconformal self-map $w$ of $\hat{\mathbb{C}}$ such that

$$
\theta(\gamma)=w \circ \gamma \circ w^{-1}, \quad \text { all } \gamma \in \Gamma .
$$

Two isomorphisms $\theta_{i}: \Gamma \rightarrow \operatorname{PSL}(2, \mathbb{C}), i=1,2$, are equivalent provided there exists an element $A \in \operatorname{PSL}(2, \mathbb{C})$ such that $\theta_{2}(\gamma)=A \circ \theta_{1}(\gamma) \circ A^{-1}$, all $\gamma \in \Gamma$. The deformation or Teichmüller space $\mathbf{T}(\Gamma)$ is the set of equivalence classes of geometric isomorphisms (see [B5, K2, Mt1]).

A quasiconformal map $w: \hat{\mathbb{C}} \rightarrow \hat{\mathbb{C}}$ is $\Gamma$-compatible if $w \circ y \circ w^{-1} \in \operatorname{PSL}(2, \mathbb{C})$ for all $\gamma \in \Gamma$. Fix three distinct limit points of $\Gamma$ (see [KM1]): $x_{1}, x_{2}, x_{3}$. A quasiconformal map $w$ is normalized if $w\left(x_{i}\right)=x_{i}$ for $i=1,2,3$. The deformation space $T(\Gamma)$ can be described as the set of restrictions to the limit set $\Lambda=\Lambda(\Gamma)$ of $\Gamma$ of the normalized $\Gamma$-compatible quasiconformal automorphisms of $\hat{\mathbb{C}}$. The topology and complex structure of $T(\Gamma)$ are completely determined by the condition that for each $x \in \Lambda$ the map

$$
\mathbf{T}(\Gamma) \ni[w] \mapsto w(x) \in \hat{\mathbb{C}}
$$

is holomorphic.

Notation. If $w$ is a $\Gamma$-compatible quasiconformal automorphism of $\hat{\mathbb{C}}$, its equivalence class in $\mathbf{T}(\Gamma)$ will be denoted by $[w]$, and the geometric isomorphism $\theta$ it induces by (4.1.1) will be denoted by $\theta_{w}$. Similarly, the equivalence class of $\theta$ in $\mathbf{T}(\Gamma)$ will be denoted by $[\theta]$.

4.2. We define the modular group of $\Gamma, \operatorname{Mod} \Gamma$, to be the group of geometric automorphisms of $\Gamma$ factored by the subgroup of inner automorphisms. We define two normalizers of $\Gamma$ :

$$
N_{\mathrm{qc}}(\Gamma)=\left\{\omega \text { quasiconformal automorphism of } \hat{\mathbb{C}} ; \omega \Gamma \omega^{-1}=\Gamma\right\},
$$

\footnotetext{
${ }^{4} \mathrm{We}$ will study this space in detail in the sequel to this paper. We will nevertheless use in this paper some of the elementary properties of this space (for example, the fact that $\mathbf{V}_{0}(\mathscr{G})$ is a complex manifold) that will be proven in the next paper in this series.

${ }^{5}$ For our purposes a "Kleinian group" always has a nonempty region of discontinuity in $\hat{\mathbb{C}}$ (these are Kleinian groups of the second kind in some modern terminology).
} 
and

$$
N(\Gamma)=\left\{\omega \in \operatorname{PSL}(2, \mathbb{C}) ; \omega \Gamma \omega^{-1}=\Gamma\right\}
$$

An element of $\operatorname{Mod} \Gamma$ is always induced by a $\theta_{\omega}$ with $\omega \in N_{\mathrm{qc}}(\Gamma) ; \theta_{\omega}$ is the trivial element of $\operatorname{Mod} \Gamma$ if and only if there is an $A \in \Gamma$ such that $\theta_{\omega}=\theta_{A}$.

The modular group, $\operatorname{Mod} \Gamma$, acts on $\mathbf{T}(\Gamma)$. If $\theta=\theta_{\omega}$ with $\omega \in N_{\mathrm{qc}}(\Gamma)$, then

$$
\theta_{\omega}^{*}([w])=\theta^{*}([w])=\left[w \circ \omega^{-1}\right], \quad[w] \in \mathbf{T}(\Gamma) .
$$

It is easily checked that for $\theta$ inner $(\omega \in \Gamma), \theta^{*}$ is trivial. Thus $\operatorname{Mod} \Gamma$ acts as a group of complex analytic automorphisms of $\mathbf{T}(\Gamma)$. The action is not always effective. The quotient space $R(\Gamma)=\mathbf{T}(\Gamma) / \operatorname{Mod} \Gamma$ represents the $\operatorname{PSL}(2, \mathbb{C})$ conjugacy classes of Kleinian groups quasiconformally equivalent to $\Gamma$, and is called the moduli or Riemann space of $\Gamma$.

Remark. Despite the clumsy appearance in (4.2.1), the inverse is necessary to insure that

$$
\theta_{\omega_{1} \circ \omega_{2}}^{*}([w])=\left[w \circ \omega_{2}^{-1} \circ \omega_{1}^{-1}\right]=\theta_{\omega_{1}}^{*}\left(\left[w \circ \omega_{2}^{-1}\right]\right)=\theta_{\omega_{1}}^{*}\left(\theta_{\omega_{2}}^{*}([w])\right)
$$

that is, to insure that the mapping from $\operatorname{Mod} \Gamma$ to $\operatorname{Aut} T(\Gamma)$, the group of complex analytic automorphisms of $\mathbf{T}(\Gamma)$, is a group homomorphism. We can rewrite (4.2.1) as (on the level of geometric isomorphisms)

$$
\theta_{\omega}^{*}([\theta])=\left[\theta \circ \theta_{\omega^{-1}}\right], \quad[\theta] \in \mathbf{T}(\Gamma) .
$$

We note that $\omega$ induces the automorphism $\tilde{\theta}_{\omega}=\theta \circ \theta_{\omega} \circ \theta^{-1}$ of $\theta(\Gamma)$. Observe that

$$
\theta \circ \theta_{\omega}^{-1}=\theta_{\omega}^{*}(\theta)=\tilde{\theta}_{\omega}^{-1} \circ \theta \text {. }
$$

4.3. If $\Gamma$ is a terminal $b$-group of type $(p, n),{ }^{6}$ then $\mathbf{T}(\Gamma)$ is a model for $\mathbf{T}(p, n)$. However, $\operatorname{Mod} \Gamma$ is not isomorphic to $\operatorname{Mod}(p, n)$, the modular group of $\mathbf{T}(p, n)$ (that is, the mapping class group of surfaces of type $(p, n))$; in particular, $\mathbf{R}(\Gamma)$ is a nontrivial covering of the Riemann space $\mathbf{R}(p, n)$. A point in $\mathbf{R}(p, n)$ represents a conformal equivalence class of surfaces of type $(p, n)$, while a point of $\mathbf{R}(\Gamma)$ represents a conformal equivalence class of a surface of type $(p, n)$ together with a maximal partition. Two distinct points $X_{1}$ and $X_{2}$ of $\mathbf{R}(\Gamma)$ project to the same point of $\mathbf{R}(p, n)$, provided there is a conformal map $h$ of the Riemann surface represented by $X_{1}$ onto the one represented by $X_{2}$. The map $h$ will not map the partition of $X_{1}$ onto that of $X_{2}$.

4.4. Let $x, x_{1}, x_{2}, x_{3}$ be four distinct fixed points of loxodromic or parabolic elements of $\Gamma$. Let $w$ be a $\Gamma$-compatible quasiconformal automorphism of $\hat{\mathbb{C}}$. Then

$$
w \mapsto \operatorname{cr}\left(w(x), w\left(x_{1}\right), w\left(x_{2}\right), w\left(x_{3}\right)\right)
$$

\footnotetext{
${ }^{6}$ See $\S 5.1$ for a definition.
} 
defines a holomorphic function

$$
f: \mathbf{T}(\Gamma) \rightarrow \mathbb{C}-\{0,1\} .
$$

If we view $\mathbf{T}(\Gamma)$ as the restrictions to $\Lambda$ of the normalized (at $\left.x_{1}, x_{2}, x_{3}\right) \Gamma$ compatible quasiconformal automorphisms of $\hat{\mathbb{C}}$, then the function $f$ defined by (4.4.1) and (4.4.2) agrees with the function defined by (4.1.2) whenever $x_{1}=\infty, x_{2}=0, x_{3}=1$.

If $\omega \in N_{\mathrm{qc}}(\Gamma)$ and $\theta=\theta_{\omega}$, then $f\left(\theta^{*}[w]\right)=f\left(\left[w \circ \omega^{-1}\right]\right)$; in particular, if both $w$ and $\omega$ are normalized at $x_{1}, x_{2}, x_{3}$, then $f\left(\theta^{*}[w]\right)=$ $\operatorname{cr}\left(w\left(\omega^{-1}(x)\right), x_{1}, x_{2}, x_{3}\right)$, and if also $x_{1}=\infty, x_{2}=0, x_{3}=1$, then $f\left(\theta^{*}[w]\right)$ $=w\left(\omega^{-1}(x)\right)$.

We shall be particularly interested in describing functions that have nice invariance properties under subgroups of $\operatorname{Mod} \Gamma$ (see $\S 7.3$ ).

4.5. We have seen that $\operatorname{Mod} \Gamma \cong N_{\mathrm{qc}}(\Gamma) / \Gamma$. To define $\operatorname{Mod}(p, n)$, it is convenient to consider a finitely generated Kleinian group $\Gamma$ with a simply connected invariant component $\Delta$. We define

$$
N_{\mathrm{qc}}(\Gamma, \Delta)=\left\{\omega \text { quasiconformal automorphism of } \Delta ; \omega \Gamma \omega^{-1}=\Gamma\right\},
$$

and

$$
\operatorname{Mod}(\Gamma, \Delta)=N_{\mathrm{qc}}(\Gamma, \Delta) / \Gamma .
$$

Then $\operatorname{Mod}(\Gamma, \Delta) \supset \operatorname{Mod} \Gamma$, and this bigger group acts on $T(\Gamma, \Delta)$, the image in $T(\Gamma)$ of the geometric isomorphisms that are conformal outside of $\Delta$, as follows. Let $\omega \in N_{\mathrm{qc}}(\Gamma, \Delta)$ and let $w$ be a $\Gamma$-compatible automorphism of $\hat{\mathbb{C}}$ that is conformal off $\Delta$. Then we define

$$
\omega^{*}([w])=[W]
$$

where $W$ is a quasiconformal automorphism of $\hat{\mathbb{C}}$ such that

$$
w \circ \omega^{-1} \circ W^{-1} \mid W(\Delta) \text { and } W \mid(\hat{\mathbb{C}}-\Delta)
$$

are conformal. One must check that $(4.5 .1)$ is well defined.

If $\Gamma$ is a terminal $b$-group and $\omega \in N_{\mathrm{qc}}(\Gamma)$, then the above definition agrees with (4.2.1). If $\Gamma$ is also torsion free, then $\operatorname{Mod}(\Gamma, \Delta)$ is a model for $\operatorname{Mod}(p, n)$.

4.6. Let $\Gamma$ be a torsion free terminal $b$-group with invariant component $\Delta$. We pick three limit points $x_{1}, x_{2}, x_{3} \in \Lambda$ for normalization of quasiconformal maps. Then

$$
\mathscr{F}(\Gamma)=\{(\tau, z) \in \mathbf{T}(\Gamma) \times \hat{\mathbb{C}} ; \tau=[w], \quad z \in w(\Delta)\}
$$

is a model for the Bers fiber space [B6]. The group $\Gamma$ acts on $\mathscr{F}(\Gamma)$ by

$$
\gamma([w], z)=\left([w], w \circ \gamma \circ w^{-1}(z)\right) .
$$

Note that in the last two equations $w$ is a normalized $\Gamma$-compatible quasiconformal map. The quotient space $\mathbf{V}(\Gamma)=\mathscr{F}(\Gamma) / \Gamma$ is a model for $\mathbf{V}^{\prime}(p, n)$, 
the punctured Teichmüller curve, it comes equipped with a natural or canonical projection $\pi_{\Gamma}: \mathbf{V}(\Gamma) \rightarrow \mathbf{T}(\Gamma)$ induced by the projection of $\mathscr{F}(\Gamma)$ onto $\mathbf{T}(\Gamma)$. For details see, for example, [K4].

\section{TORSION FREE TERMINAL $b$-GROUPS}

This section summarizes the structure theorems of Maskit [Mt2] that are needed in $\S 7$. We emphasize the relationship between torsion free regular $b$ groups and admissible graphs.

5.1. Let $S$ be a Riemann surface of finite analytic type $(p, n)$ with $v=2 p-$ $2+n>0$. Let $\Sigma=\left\{a_{1}, \ldots, a_{d}\right\}, d=3 p-3+n$, be a maximal partition on $S^{7}$ (exclude from now on the case $(p, n)=(0,3)$ where $\Sigma$ is empty). Let $S_{0}=S-\Sigma=S^{1} \cup \cdots \cup S^{v}$ be the decomposition of the complement on $S$ of the partition curves into the parts of $S$. Let $\mathscr{G}$ be the graph corresponding to $(S, \Sigma)$ as in $\S 3.2$. We henceforth use the notation and conventions from that subsection; in particular, we given $\mathscr{G}$ a semicanonical ordering for its edges.

A Kleinian group $\Gamma$ is a function group if it has an invariant component $\Delta$; it is a $b$-group if $\Delta$ is also simply connected. Assume that $\Gamma$ is a torsion free $b$-group. The type $(p, n)$ of $\Delta / \Gamma$ is also called the type of $\Gamma$. The torsion free $b$-group is called terminal if $(\Omega-\Delta) / \Gamma$ is a union of $v$ thrice punctured spheres, where $\Omega=\Omega(\Gamma)$ is the region of discontinuity of $\Gamma$. In this case, there are $d$ simple disjoint curves $\tilde{a}_{1}, \ldots, \tilde{a}_{d}$ in $\Delta$; each curve $\tilde{a}_{j}$ is precisely invariant under an accidental parabolic cyclic subgroup $\left\langle A_{j}\right\rangle$ in $\Gamma$ (the parabolic element $A_{j}$ does not represent punctures on $\Delta / \Gamma$ ). It involves no loss of generality to assume that the curve $\tilde{a}_{j}$ (and hence also its projection to $\Delta / \Gamma$ ) is a geodesic in the Poincare metric on $\Delta(\Delta / \Gamma)$. We say that $\Gamma$ represents the pair $(S, \Sigma)$, as in $[\mathrm{K} 6, \S 1]$ for signature $(p, n ; \infty, \ldots, \infty)$, if $S \cong \Delta / \Gamma$ (as Riemann surfaces) and $\pi\left(\tilde{a}_{j}\right)$ is freely homotopic to $a_{j}, j=1, \ldots, d$, where $\pi: \Delta \rightarrow \Delta / \Gamma$ is the natural projection. The accidental parabolic element $A_{j} \in \Gamma$ is said to correspond to the partition curve $a_{j} \in \Sigma$. By changing the curves $\tilde{a}_{j}$, we may and do assume that $\pi\left(\tilde{a}_{j}\right)=a_{j}$. We shall say that the partition $\Sigma$ and the torsion free terminal $b$-group $\Gamma$ are of graph type $\mathscr{G}$. The graph type is a complete quasiconformal invariant for such groups (see Maskit [Mt2]). A torsion free terminal $b$-group $\Gamma$ with invariant component $\Delta$ is of graph type $\mathscr{G}$ if and only if there exists a homeomorphism of $\Delta / \Gamma$ onto (the topological surface represented by) $\mathscr{G}$ that maps the geodesics on $\Delta / \Gamma$ determined by the accidental parabolic elements of $\Gamma$ onto partition curves on $\mathscr{G}$ determined by its edges.

Start with the family of disjoint loops (known as the structure loops [Mt2]), $\pi^{-1}\left(a_{1} \cup \cdots \cup a_{d}\right)=\tilde{\Sigma}$, that partition $\Delta_{0}=\Delta-\tilde{\Sigma}$ into a disjoint union of structure regions. Each structure region covers a part of $S$. The stabilizer of each structure region is a triangle group (known as a structure subgroup of

\footnotetext{
${ }^{7}$ Note that $S$ is $S_{0}$ of $[\mathrm{K} 6, \S 1]$.
} 
$\Gamma)$. Two distinct structure subgroups of $\Gamma$ intersect trivially or in a common accidental parabolic cyclic subgroup. In the former case the closures of the corresponding structure regions (in $\Delta$ ) are disjoint; in the latter, their closures intersect in a structure loop.

The structure loops are in one-to-one canonical correspondence with the maximal cyclic accidental parabolic subgroups of $\Gamma$. Each structure loop is on the boundary of exactly two structure regions; hence each accidental parabolic cyclic subgroup is in exactly two structure subgroups. These two structure subgroups generate a terminal regular $b$-group of type $(0,4)$ if they are not conjugate in $\Gamma$ and the two groups are contained in a terminal regular $b$-group of type $(1,1)$ if they are conjugate in $\Gamma$.

5.2. We proceed to choose convenient representatives of the $v$ conjugacy classes of structure subgroups, and to describe the tessalation of $\Delta$ by the structure regions. If $\mathscr{G}$ has more than one vertex, then for $1 \leq j \leq v-1, a_{j}$ is the common boundary curve of $S^{j+1}$ and $S^{\tau(j)}$ for some. $\tau(j)$ with $1 \leq \tau(j) \leq j$ (notice that $\tau(1)=1$ ). Let $\tilde{S}_{1}$ be any structure region covering $S^{1}$. For $j=1, \ldots, v-1$, we choose a structure region $\widetilde{S}_{j+1}$ covering $S^{j+1}$ so that $\widetilde{S}_{j+1}$ and $\widetilde{S}_{\tau(j)}$ have a structure loop (covering $a_{j}$ ) as a common boundary. For $j=v, \ldots, d$, a structure loop covering $a_{j}$ is on the boundary of some region $\widetilde{S}_{\tau(j)}$ with $1 \leq \tau(j) \leq v$. Choose $\widetilde{S}_{j+1}$ to be the adjacent structure region whose common boundary with $\widetilde{S}_{\tau(j)}$ covers $a_{j}$. For $j=d+1, d+2, \ldots$, pick a new region $\widetilde{S}_{j+1}$ to be adjacent to some $\tilde{S}_{\tau(j)}$ with $\tau(j) \leq j$. In this manner we enumerate all the structure regions of $\Gamma$; that is, $\Delta=\bigcup_{j=1}^{\infty} \mathrm{cl} \widetilde{S}_{j}$, where cl stands for closure with respect to $\Delta$.

Let $F_{j}$ be the stabilizer of $\tilde{S}_{j}$. Then by our construction $F_{j+1} \cap F_{\tau(j)}=$ $\left\langle A_{j}\right\rangle, j=1,2, \ldots$, where $A_{j}$ is an accidental parabolic element, and every accidental parabolic element in $\Gamma$ is a power of some $A_{j}$. The collection $\left\langle A_{1}\right\rangle, \ldots,\left\langle A_{d}\right\rangle$ is a maximal set of nonconjugate cyclic accidental parabolic subgroups of $\Gamma$; the accidental parabolic element $A_{j} \in \Gamma$ corresponds to the edge $a_{j} \in \mathscr{G}$. The collection $F_{1}, \ldots, F_{v}$ is a maximal set of nonconjugate structure subgroups of $\Gamma$; the structure subgroup $F_{k}$ of $\Gamma$ corresponds to the vertex $S^{k}$ on $\mathscr{G}$. The group $\Gamma_{0}$ generated by $F_{1}, \ldots, F_{v}$ is a terminal regular $b$-group of type $(0,2 p+n)$; it is obtained by $v-1$ AFP constructions. The group $\Gamma$ is obtained from $\Gamma_{0}$ by $p$ HNN-extensions. See [Mt2] for details.

5.3. We now describe convenient representatives for the modular subgroups (see [K6, §2]) of $\Gamma$. A modular subgroup can be defined abstractly as a subgroup of $\Gamma$ that is a terminal $b$-group of type $(0,4)$ or $(1,1)$. A modular region is a domain in $\Delta$ bounded by structure loops and stabilized by a modular subgroup. A modular region covers a modular part of $S$, that is, a subsurface of type $(0,4)$ or $(1,1)$ bounded by partition curves. 
The modular parts of $S$ are in one-to-one canonical correspondence with the partition curves in $\Sigma$. The modular part $T_{j}$ corresponding to the curve $a_{j}$ is the connected component of $S-\bigcup_{i \neq j} a_{i}$ containing $a_{j}$.

A modular part of type $(1,1)$ is an elliptic end of $S$; a modular part of type $(0,4)$ is a spherical end provided it has two or more punctures (that is, at most two of its boundary components are curves in $\Sigma$ ). An end is either a spherical or an elliptic end.

Two distinct modular subgroups intersect trivially, in a cyclic accidental parabolic subgroup, or in a structure subgroup. The three cases correspond to the closures in $\Delta$ of the corresponding modular regions being disjoint, intersecting in a structure loop, or intersecting in the closure of a structure region.

Every accidental parabolic subgroup of $\Gamma$ is an accidental parabolic subgroup of exactly one modular subgroup. Hence we can define $G_{j}$ to be the modular subgroup of $\Gamma$ in which $\left\langle A_{j}\right\rangle$ is an accidental parabolic subgroup. It follows that for $j=1, \ldots, v-1, G_{j}=F_{j+1}{ }^{\left.{ }_{\left(A_{j}\right\rangle}\right\rangle} F_{\tau(j)}$ (that is, $G_{j}$ is the amalgamated free product (AFP) of $F_{j+1}$ and $F_{\tau(j)}$ across the common cyclic subgroup $\left\langle A_{j}\right\rangle$ ). For $j=v, \ldots, d, G_{j}$ is the modular subgroup containing $F_{j+1}$ and $F_{\tau(j)}$; it is an HNN-extension of $F_{\tau(j)}$ by an element $C_{j}^{-1}$ conjugating $A_{j} \in F_{\tau(j)}$ to $C_{j}^{-1} \circ A_{j} \circ C_{j} \in F_{\tau(j)}$ if $F_{j+1}$ and $F_{\tau(j)}$ are conjugate in $\Gamma$ and it is an AFP of $F_{j+1}$ and $F_{\tau(j)}$ across $\left\langle A_{j}\right\rangle$ if $F_{j+1}$ and $F_{\tau(j)}$ are not conjugate in $\Gamma$.

The modular subgroups $G_{1}, \ldots, G_{d}$ are a maximal collection of $\Gamma$-inequivalent modular subgroups of $\Gamma$; the modular subgroup $G_{j} \subset \Gamma$ corresponds to the edge $a_{j}$ on $\mathscr{G}$. Our numbering agrees with that in [K6, §2]. Note that a structure subgroup is contained in exactly one, two or three modular subgroups of $\Gamma$, depending on the number of distinct partition curves bounding the part of $S$ corresponding to the structure subgroup.

5.4. For each type $(p, n)$ with $v>0$ there is a natural equivalence between:

(A) maximal partitions $\Sigma$ of a topological surface of type $(p, n)$,

(B) admissible graphs $\mathscr{G}$ of type $(p, n)$, and

(C) quasiconformal equivalence classes $\Gamma$ of torsion free terminal $b$-groups of type $(p, n)$.

Assume that a graph $\mathscr{G}$ is an HNN-extension of $\mathscr{G}^{\prime}$. If $\Gamma$ and $\Gamma^{\prime}$ are Kleinian groups of graph type $\mathscr{G}$ and $\mathscr{G}^{\prime}$, respectively, then $\Gamma$ is an HNNextension of a quasiconformal conjugate of $\Gamma^{\prime}$. Similarly, if $\Gamma$ is an AFP of $\mathscr{G}^{\prime}$ and $\mathscr{G}^{\prime \prime}$, and if $\Gamma, \Gamma^{\prime}$ and $\Gamma^{\prime \prime}$ are of graph type $\mathscr{G}, \mathscr{G}^{\prime}$ and $\mathscr{G}^{\prime \prime}$, respectively, then $\Gamma$ is an AFP of quasiconformal conjugates of $\Gamma^{\prime}$ and $\Gamma^{\prime \prime}$. For details see $\S 7.5$, where a stronger theorem is proved.

\section{ONE-DIMENSIONAL DEFORMATION SPACES}

In this section we study in detail the modular subgroups of torsion free terminal $b$-groups; that is, terminal $b$-groups of types $(0,4)$ and $(1,1)$. We determine the plumbing constructions that yield the surfaces represented by such groups. 


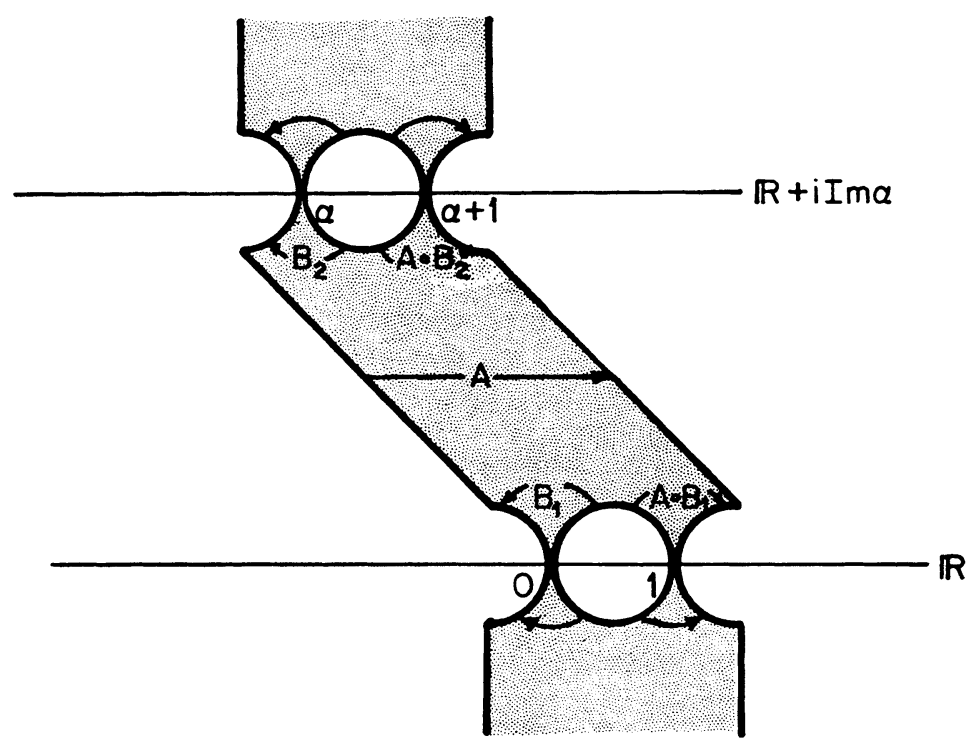

FIGURE 7. A fundamental domain for a terminal group of type $(0,4)$.

6.1. Let $\Gamma$ be a torsion free terminal $b$-group of type $(0,4)$; it is an AFP of two torsion free triangle groups $F_{1}$ and $F_{2}$ across a common cyclic parabolic subgroup $\langle A\rangle, A \in F_{1} \cap F_{2}$ (see Figure 7). The augmented graph corresponding to the group $\Gamma$ is shown in Figure 6. The group $\Gamma$ represents, on its invariant component $\Delta$, a sphere with four punctures and a partition curve $\tilde{a} \quad(A \in \Gamma$ corresponds to the curve $\tilde{a}$ ). Let $S^{1}$ and $S^{2}$ be the parts of $\Delta / \Gamma-\{\tilde{a}\}$. We orient $\tilde{a}$ so that $S^{1}$ lies to the right of $\tilde{a}$. We assume that $F_{j}$ is the structure subgroup corresponding to $S^{j}$, for $j=1,2$. Without loss of generality $F_{1}=F$ of $\S 1.2$ and $(A, B)$ are canonical generators for $F_{1}$. Note that the orientation assumptions guarantee that $S^{1}$ is represented by the action of $F_{1}$ on $\mathbb{H}^{2}$ (and not the lower half-plane $\mathbb{H}_{*}^{2}$ ). We relabel $B=B_{1}$ in (1.2.2).

Choose $B_{2} \in F_{2}$ so that $\left(A, B_{2}\right)$ are canonical generators for $F_{2}$. Then in $\mathrm{SL}(2, \mathbb{C})$,

$$
\begin{gathered}
B_{2}=\left[\begin{array}{ll}
a & b \\
c & d
\end{array}\right], \quad a d-b c=1, \\
\operatorname{tr} B_{2}=a+d=-2, \quad \operatorname{tr}\left(A \circ B_{2}\right)=-a-2 c-d=-2 .
\end{gathered}
$$

It follows that $c=2, d=-2-a$ and $2(a+b)+a^{2}=-1$. Because $S^{2}$ lies to the left of the curve $\tilde{a}$ (Figure 7), the fixed point $\alpha=f\left(B_{2}\right)$ of $B_{2}$ must be in $\mathbb{H}^{2}$. Then

$$
B_{2}=\left[\begin{array}{cc}
-1+2 \alpha & -2 \alpha^{2} \\
2 & -1-2 \alpha
\end{array}\right]=B_{\alpha} .
$$

The reader should compare this formula with the formula for $C$ in $\S 1.3$. 
Since $\mathbb{H}^{2}+i(\operatorname{Im} \alpha)$ is precisely invariant under $F_{2}$ in $\Gamma$, we conclude that every $\gamma \in F_{1}, \gamma \notin\langle A\rangle$, maps this half-plane into the strip

$$
\{z \in \mathbb{C} ; 0<\operatorname{Im} z<\operatorname{Im} \alpha\} .
$$

If $\gamma=\left[\begin{array}{ll}a & b \\ c & d\end{array}\right], \gamma \in F_{1}-\langle A\rangle$, then $|c| \geq 2$,

$$
\operatorname{Im} \gamma(z)=\frac{\operatorname{Im} z}{(c(\operatorname{Re} z)+d)^{2}+c^{2}(\operatorname{Im} z)^{2}} \leq \frac{1}{c^{2}(\operatorname{Im} z)}
$$

and

$$
\operatorname{Im} \gamma\left(-\frac{d}{c}+i(\operatorname{Im} z)\right)=\frac{1}{c^{2}(\operatorname{Im} z)} .
$$

It follows that $\operatorname{Im} \alpha>\frac{1}{2}$. It is easily seen that whenever $\operatorname{Im} \alpha>1$, then Maskit's [Mt2] combination theorem applies and the AFP of $F_{1}$ and $F_{2}$ across $\langle A\rangle$ is a terminal $b$-group of type $(0,4)$. We have shown that in the $\alpha$ coordinate,

$$
\{\alpha \in \mathbb{C} ; \operatorname{Im} \alpha>1\} \subset \mathbf{T}(0,4) \subset\left\{\alpha \in \mathbb{C} ; \operatorname{Im} \alpha>\frac{1}{2}\right\} .
$$

Notation. (1) The group $\Gamma$ constructed above will be denoted by $\Gamma_{1}(\alpha)$; its invariant component, by $\Delta(\alpha)$.

(2) We will use the following rule for picking parabolic generators: the puncture corresponding to the given generator lies to the left of the horocircles determined by the generator (see §7.4). In $\S 12.1$, we introduce a notational convention for describing triangle groups and their canonical generators. Using this notation, $\Gamma_{1}(\alpha)$ is the AFP of $F(\infty, 0,1)$, whose canonical generators are $(A, B)$, with $F(\infty, \alpha, \alpha-1)$, whose canonical generators are $\left(A^{-1}, B_{\alpha}^{-1}\right)$, across the cyclic parabolic subgroup $\langle A\rangle$.

Remarks. (1) We have reproven the result obtained in [K6] that $\operatorname{tr}\left(B_{2}^{-1} \circ B_{1}\right)$ is a global coordinate on $\mathrm{T}(0,4)$, since $\alpha \mapsto 2+4 \alpha^{2}$ is injective on $\mathbf{T}(0,4)$. Note, however, that $\operatorname{tr}^{2}\left(B_{2}^{-1} \circ B_{1}\right)$ is not a global coordinate on $\mathbf{T}(0,4)$.

(2) Formula (6.1.1) is equally valid for $\alpha \in \mathbb{H}_{*}^{2}$. The group $\Gamma_{1}(\alpha)$ so obtained is (for appropriate values of $\alpha$ ), of course, a terminal $b$-group of type $(0,4)$. Its invariant component is a subset of $\mathbb{H}_{*}^{2}$ and the punctures lie "on the wrong side" of the horocircles. Consider the motion $E=\left[\begin{array}{cc}1 & -\alpha \\ 0 & 1\end{array}\right] \in \operatorname{PSL}(2, \mathbb{C})$. The group $E \Gamma_{1}(\alpha) E^{-1}$ is the AFP of $E F(\infty, 0,1) E^{-1}=F(\infty,-\alpha,-\alpha+1)$ and $E F(\infty, \alpha, \alpha-1) E^{-1}=F(\infty, 0,-1)$ across $\langle A\rangle$. Hence $E \Gamma_{1}(\alpha) E^{-1}=$ $\Gamma_{1}(-\alpha)$. The groups $\Gamma_{1}(\alpha)$ and $\Gamma_{1}(-\alpha)$ represent the same surface $S$. However, in the natural orientation for the curve $\tilde{a}$ (corresponding to $A$ ), the part represented by $F(\infty, 0,1)$ lies on the right of the curve $\tilde{a}$ in $\Gamma_{1}(\alpha)$ and to the left in $\Gamma_{1}(-\alpha)$.

(3) The first inclusion in (6.1.2) is sharp since $i \notin \mathbf{T}(0,4)$. For $\alpha=i$, $\operatorname{tr}\left(B_{2}^{-1} \circ B_{1}\right)=-2$ and hence $\Gamma_{1}(i)$ is not a terminal $b$-group of type $(0,4)$. It is also easy to see that $-\frac{1}{2}+r i \in \mathbf{T}(0,4)$ for all $r>\sqrt{3} / 2$, but $-\frac{1}{2}+(\sqrt{3} / 2) i \notin$ 
$\mathbf{T}(0,4)$ (because $A^{1 / 2} \circ B_{2} \circ B_{1}^{-1} \in N\left(\Gamma_{1}(\alpha)\right)$, as shown in $\S 8.1$, and its trace is -2 for this value of $\alpha$ ).

(4) Note that $\Gamma_{1}(1+\alpha)=\Gamma_{1}(\alpha)$.

(5) Fix a group $\Gamma_{0}=\Gamma_{1}\left(\alpha_{0}\right)$; for example, take $\alpha_{0}=2 i$. We view $T(\Gamma)$ as a model for $\mathbf{T}(0,4)$. Then $\alpha=w\left(\alpha_{0}\right)$, where $w$ is a normalized (at 0,1 and

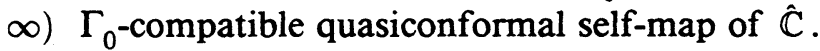

6.2. For the sake of convenience, we label the puncture on $\mathbb{H}^{2} / F$ determined by the parabolic fixed point $x \in \mathbb{R} \cup\{\infty\}$ by the symbol $\hat{x}$. We will use the same convention for punctures on other surfaces.

We claim that $\Delta(\alpha) / \Gamma_{1}(\alpha)$ is obtained by a $z w=t$ construction from $\mathbb{H}^{2} / F_{1}$ and $\left(\mathbb{H}_{*}^{2}+i(\operatorname{Im} \alpha)\right) / F_{2}$. Let $z$ be the horocyclic coordinate on $\mathbb{H}^{2} / F_{1}$ at $\hat{\infty}$ relative to $\hat{0}$, and let $w$ be the horocyclic coordinate on $\left(\mathbb{H}_{*}^{2}+i(\operatorname{Im} \alpha)\right) / F_{2}$ at $\hat{\infty}$ relative to $\hat{\alpha}$. Then $z=e^{\pi i \zeta}, w=e^{-\pi i(\zeta-\alpha)}$, for a nonempty open subset of $\Delta(\alpha)$. Hence $z w=e^{\pi i \alpha}=t$ (note that if $\operatorname{Im} \alpha>1$ and if we set $r=e^{-\pi(\operatorname{Im}(\alpha-1 / 2))}=e^{\pi / 2}|t|$, then we are exactly in the situation described in $\S 2.2)$ and $\Delta(\alpha) / \Gamma_{1}(\alpha)=S_{t}$. For $\operatorname{Im} \alpha>1$, the annulus $\mathscr{A}_{t}$ on $S_{t}$ is the image in $\Delta(\alpha) / \Gamma_{1}(\alpha)$ of the strip

$$
\left\{\zeta \in \mathbb{C} ; \frac{1}{2}<\operatorname{Im} \zeta<\operatorname{Im} \alpha-\frac{1}{2}\right\},
$$

and the central curve on $\mathscr{A}_{t}$ is the image of the line $\left\{\zeta \in \mathbb{C} ; \operatorname{Im} \zeta=\frac{1}{2} \operatorname{Im} \alpha\right\}$.

Remarks. (1) In the above plumbing construction we have used the cyclic ordering of the punctures on $\mathbb{H}^{2} / F_{1}$ and $\left(\mathbb{H}_{*}^{2}+i(\operatorname{Im} \alpha)\right) / F_{2}$ specified by $\hat{\infty}, \hat{0}, \hat{1}$, and $\hat{\infty}, \hat{\alpha}, \widehat{\alpha-1}$, respectively.

(2) From $\Gamma_{1}(\alpha+1)=\Gamma_{1}(\alpha)$ (see Remark (4) in $\S 6.1$ ), we conclude (once again) that $S_{-t}$ is conformally equivalent to $S_{t}$ (see also $\S 2.3$ ).

Let $\Gamma$ be an arbitrary torsion free terminal $b$-group of type $(0,4)$ with invariant component $\Delta$. Let $A \in \Gamma$ be a primitive accidental parabolic element. Let $F_{1}$ and $F_{2}$ be the two structure subgroups of $\Gamma$ that contain $\langle A\rangle$. We choose the indices so that the part of $\Delta / \Gamma$ corresponding to $F_{1}$ lies to the right of the oriented partition curve determined by $A$. For $j=1,2$, choose $B_{j} \in F_{j}$ so that $\left(A, B_{1}\right)$ are canonical generators for $F_{1}$ and $\left(A^{-1}, B_{2}^{-1}\right)$ are canonical generators for $F_{2}$. Let $a=f(A), b_{j}=f\left(B_{j}\right), j=1,2$. Then

$$
F_{1}=F\left(a, b_{1}, c_{1}\right), \quad c_{1}=A^{1 / 2}\left(b_{1}\right), \quad F_{2}=F\left(a, b_{2}, c_{2}\right), \quad c_{2}=A^{-1 / 2}\left(b_{2}\right) .
$$

We define

$$
\tau=\tau(\Gamma)=\operatorname{cr}\left(b_{2}, a, b_{1}, c_{1}\right) \text { and } t=t(\Gamma)=e^{\pi i \tau(\Gamma)} .
$$

We call $\tau(\Gamma)$ a horocyclic coordinate for the group $\Gamma$ and $t(\Gamma)$ a plumbing parameter for $\Gamma$.

Theorem. We have $0<|t|<e^{-\pi / 2}$. The complex number $t^{2}$ is a complete $\operatorname{PSL}(2, \mathbb{C})$ conjugacy class invariant of $\Gamma ;$ further $\Delta / \Gamma \cong S_{t}$. The plumbing 
parameter $t$ is independent of choices of generators of the structure subgroups of $\Gamma$, except that it may be replaced by $-t$. The horocyclic coordinate $\tau$ is determined modulo $\mathbb{Z}$ by $\Gamma$.

Proof. The quantities $\tau$ and $t$ are conjugation invariants. Hence we may assume $F_{1}=F(\infty, 0,1)$. The results of $\S 6.1$ show that $B_{2}=B_{\tau}$ of (6.1.1). Thus $\operatorname{Im} \tau>\frac{1}{2}$. The most general pair $\left(A^{-1}, B_{2}^{*}\right)$ of canonical generators for $F_{2}$ is given by $B_{2}^{*}=A^{-n / 2} \circ B_{\alpha} \circ A^{n / 2}$ as a result of Lemma 1.3. Since $A^{-n / 2} \circ B_{\tau} \circ A^{n / 2}=B_{\tau-n}, \alpha$ is uniquely determined modulo $\mathbb{Z}$ by $\Gamma$. Since $\Gamma_{1}(\alpha+1)=\Gamma_{1}(\alpha), t^{2}$ is a conjugacy class invariant for $\Gamma$. We shall show in $\S 8.2$ that $t^{2}$ is a complete conjugacy class invariant for $\Gamma$ (that is, $t^{2}\left(\Gamma_{1}\right)=t^{2}\left(\Gamma_{2}\right)$ if and only if $\Gamma_{2}$ is conjugate to $\Gamma_{1}$ ). The other claims in the theorem have already been established.

The theorem shows that the (not necessary tame) plumbing construction (see $\S 2.3$ ) depends only on the gluing parameter $t$ (and not the annuli $\mathscr{A}_{1}$ and $\mathscr{A}_{2}$ ). Further, for surfaces of type $(0,4), S_{t}$ is unambiguously defined; it is independent of the choice of local coordinates since $S_{t}$ is conformally equivalent to $S_{-t}$.

6.3. Let $\Gamma$ be a torsion free terminal $b$-group of type $(1,1)$. Then $\Gamma$ is an HNN-extension of a triangle group $F$ by an element $C \in \operatorname{PSL}(2, \mathbb{C}$ ) (see Figure 8). Let (as above) $\Delta$ be the invariant component of $\Gamma, \tilde{a}$ the partition curve on $\Delta / \Gamma$, and $S^{1}=\Delta / \Gamma-\{\tilde{a}\}$. Then $F$ is a structure subgroup of $\Gamma$ corresponding to the (single) part $S^{1}$. We let $A \in F$ correspond to the curve $\tilde{a}$. By conjugation, we may take $F$ to be the group described in $\S 1.2$ and $(A, B)$ to be canonical generators for $F$. Further, we may assume that $B^{-1} \circ A^{-1}$ represents the puncture on $\Delta / \Gamma$. Thus $A$ is conjugate to $B^{ \pm 1}$ in $\Gamma$ (but not in $F$ ). We choose an orientation for the curve $\tilde{a}$ so that the puncture (boundary component) on $S^{1}$ corresponding to the element $A\left(B^{-1}\right)$ lies to the left (right) of the horocircles determined by this motion. It follows that $A$ is conjugate to $B^{-1}$ in $\Gamma$. We take $C$ to satisfy

$$
C \circ B^{-1} \circ C^{-1}=A \text {. }
$$

(Note that the intersection number of the curves $\tilde{a}$ and $\tilde{c}$ corresponding to $A$ and $C$ must be +1.$)$ Writing $C=\left[\begin{array}{ll}a & b \\ c d & d\end{array}\right], a d-b c=1$, we see from (1.2.2) and (6.3.1) that

$$
\left[\begin{array}{ll}
a & b \\
c & d
\end{array}\right]\left[\begin{array}{cc}
-1 & 0 \\
-2 & -1
\end{array}\right]= \pm\left[\begin{array}{cc}
-1 & -2 \\
0 & -1
\end{array}\right]\left[\begin{array}{ll}
a & b \\
c & d
\end{array}\right]
$$

It follows that the plus sign holds in (6.3.2), and thus (6.3.1) is valid in $\operatorname{SL}(2, \mathbb{C})$. Also $d=0, b=c, b^{2}=-1$. We let $\tau=-i(\operatorname{tr} C)$ and conclude that

$$
C=C_{\tau}=\left[\begin{array}{cc}
i \tau & i \\
i & 0
\end{array}\right]=i\left[\begin{array}{ll}
\tau & 1 \\
1 & 0
\end{array}\right] \text {. }
$$



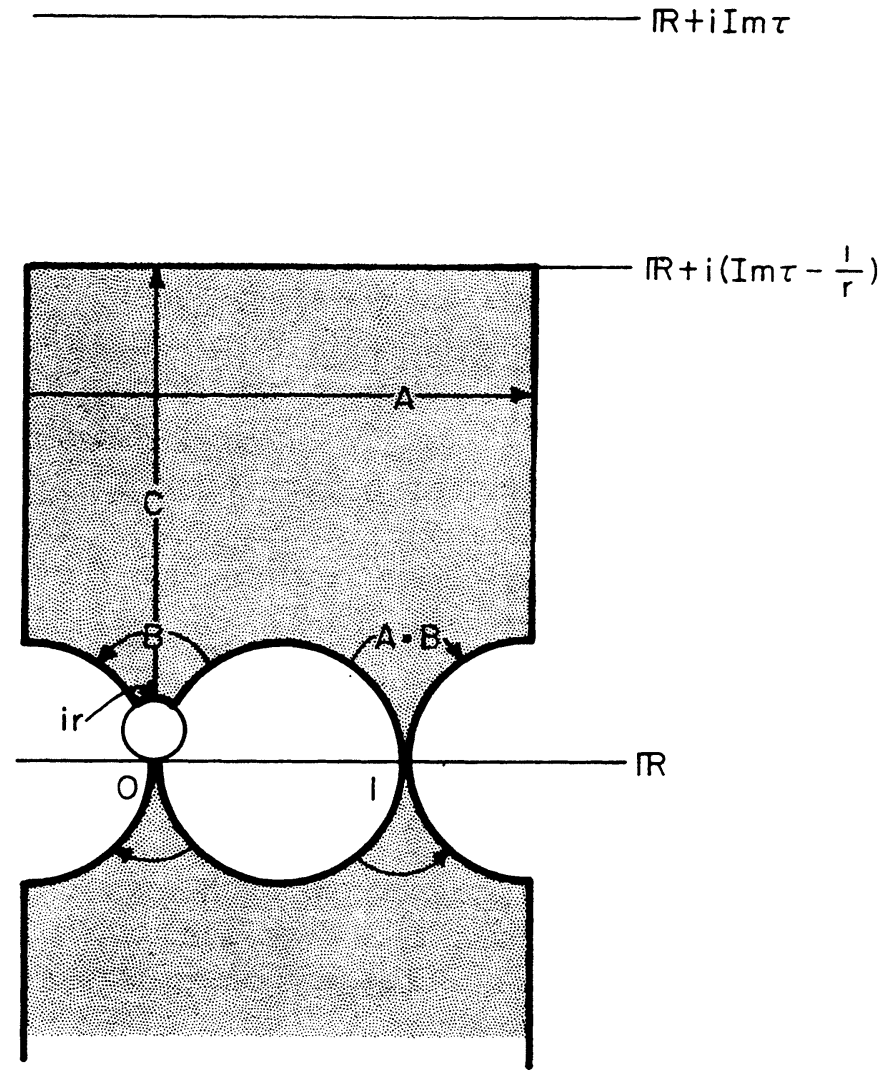

FIGURE 8. A fundamental domain for a terminal group of type $(1,1)$.

We have reproven that $C$ is uniquely determined by its trace [K6]. The orientation choices on the curves $\tilde{a}$ and $\tilde{c}$ force the invariant component $\Delta$ of $\Gamma$ to be a subset of $\mathbb{H}^{2}$. It follows that $\mathbb{H}_{*}^{2}$ is precisely invariant under $F$ in $\Gamma$. Hence $C\left(\mathbb{H}_{*}^{2}\right) \subset \mathbb{H}^{2}$. From this observation, $\operatorname{Im} C(i y)>0$ for all $y<0$, we conclude that $\operatorname{Im} \tau>0$. We shall see shortly that we actually have a better estimate $\left(\operatorname{Im} \tau>\frac{1}{2}\right)$, and in $\S 8.6$ we will obtain a further improved bound $(\operatorname{Im} \tau>1)$. Note that $C$ maps a horodisc $U_{0}$ of the point 0 onto the complement of a horodisc $U_{\infty}$ of the point $\infty$. The map $C$ takes the horocircle

$$
\left\{z \in \mathbb{C} ;\left|z-\frac{r}{2} i\right|=\frac{r}{2}\right\}, \quad r>0,
$$

(about 0 ) onto the horocircle $\{z \in \mathbb{C} ; \operatorname{Im} z=-1 / r+\operatorname{Im} \tau\}$ (about $\infty$ ). These two horocircles are disjoint if and only if $r+1 / r<\operatorname{Im} \tau$. The minimum value of $r+1 / r$ is 2 (at $r=1$ ). We conclude that for $\operatorname{Im} \tau>2$, the group $\Gamma$ constructed above is always Kleinian: a torsion free terminal $b$-group of type $(1,1)$. The region in $\mathbf{T}(1,1)$ given by $\operatorname{Im} \tau>2$ corresponds precisely to 
those groups $\Gamma$ that can be constructed through the use of horodiscs (rather than arbitrary horocyclic neighborhoods).

We have shown that $\mathbf{T}(1,1)$ contains the half-plane $\{\tau \in \mathbb{C} ; \operatorname{Im} \tau>2\}$ and we will see below that $\mathbf{T}(1,1)$ is contained in the half-plane $\left\{\tau \in \mathbb{C} ; \operatorname{Im} \tau>\frac{1}{2}\right\}$. We note that the group $\Gamma$ (its class in $T(1,1)$ ) is uniquely determined by $\operatorname{tr}^{2} C$ (in (6.3.3) we choose $\tau$ so that $\operatorname{tr} C=i \tau$ has negative real part).

We need an alternate description of the group $\Gamma$. Start with the canonical generators $\left(B, B^{-1} \circ A^{-1}\right)$ for $F=F(0,1, \infty)$ with the usual convention that the punctures lie to the left of the horocircles. Then $C F C^{-1}=F(\infty, \tau+1, \tau)$ has canonical generators $\left(A^{-1}, B_{\tau+1}^{-1}\right)$. The group generated by $F$ and $C F C^{-1}$ is precisely $\Gamma_{1}(\tau+1)=\Gamma_{1}(\tau)$. It follows that $\operatorname{Im} \tau>\frac{1}{2}$ (see $\S 8.6$ for an improved bound). Thus we also see that the group $\Gamma$ can be constructed from $\Gamma_{1}(\tau+1)$ by adjoining the Möbius transformation $C$ that sends 0,1 and $\infty$ to $\infty, \tau+1$ and $\tau$ (respectively).

Notation. We shall denote by $\Gamma_{2}(\tau)$ the group constructed in this paragraph for the parameter $\tau$. The invariant domain of $\Gamma_{2}(\tau)$ will be denoted by $\Delta(\tau)$.

Remarks. (1) We shall say that an AFP or HNN construction is tame if the neighborhoods involved ( $U_{0}$ and $U_{\infty}$, for example) in all the constructions are horodiscs. The tame constructions in our case correspond to the region $\{\tau \in \mathbb{C} ; \operatorname{Im} \tau>2\}$. Note that $\mathbf{T}(1,1)$ does not contain any larger half-plane, since for $\tau=2 i, \operatorname{tr} C=-2$. (The terminology corresponds to the one introduced for plumbing constructions in $\S 2.3$.)

(2) We have shown that for each $\tau \in \mathbf{T}(1,1), \Gamma_{2}(\tau) \supset \Gamma_{1}(\tau)$ (necessarily of infinite index). Conversely, given $\alpha \in \mathbf{T}(0,4)$, we can choose the unique element $C \in \operatorname{PSL}(2, \mathbb{C})$ such that

$$
C \circ B^{-1} \circ C^{-1}=A, \quad C \circ(B \circ A) \circ C^{-1}=B_{\alpha-1}, \quad C \circ A^{-1} \circ C^{-1}=B_{\alpha} .
$$

Here $B_{\alpha-1}$ is given by (6.1.1). Existence and uniqueness of $C$ is easily established (see Proposition 12.1); it must be of the form (6.3.3) with $\tau=\alpha$. The group $\Gamma_{2}(\alpha)$ generated by $\Gamma_{1}(\alpha)$ and $C$ is also generated by $F$ and $C$. It is not always Kleinian. For example, we have seen that

$$
\{\alpha \in \mathbb{R} ; \operatorname{Re} \alpha=0, \operatorname{Im} \alpha>1\} \subset \mathbf{T}(0,4) .
$$

For such $\alpha, \operatorname{tr} C=i \alpha$ is real and negative. Thus the groups $\Gamma_{2}(\alpha)$ for $\{\alpha \in$ $\mathbb{C} ; \operatorname{Re} \alpha=0,1<\operatorname{Im} \alpha \leq 2\}$ cannot correspond to points in $\mathbf{T}(1,1)$.

(3) We have produced a nontrivial holomorphic embedding $\mathbf{T}(1,1) \rightarrow$ $\mathbf{T}(0,4)$. It is known that these spaces are complex analytically equivalent. In $\S 8.6$ we will produce such an equivalence.

(4) Formula (6.3.3) is, of course, also valid for $\tau \in \mathbb{H}_{*}^{2}$. If $\Gamma_{2}(\tau)$ is the group so produced, then $J \Gamma_{2}(\tau) J^{-1}=\Gamma_{2}(\bar{\tau})$, where $J$ is the anticonformal involution $J(z)=\bar{z}$. However, the above formula does not reveal the entire picture. Consider the conformal involution $E=i\left[\begin{array}{ll}0 & 1 \\ 1 & 0\end{array}\right]$ that maps $\mathbb{H}^{2}$ to $\mathbb{H}_{*}^{2}$. 
It is easily shown that $E \Gamma_{2}(\tau) E^{-1}=\Gamma_{2}(-\tau)$ (note that $E \circ A \circ E^{-1}=B^{-1}$, $E \circ B \circ E^{-1}=A^{-1}, E \circ C_{\tau} \circ E^{-1}=C_{-\tau}^{-1}$, where $C_{\tau}$ is given by (6.3.3)).

(5) We have seen that orientation considerations force our conjugation to satisfy (6.3.1). We could consider the case $C \circ B^{-1} \circ C^{-1}=A^{-1}$ and conclude that $C=\left[\begin{array}{cc}i \tau & 1 \\ -1 & 0\end{array}\right], \operatorname{tr} C=i \tau$. Thus $C$ maps the horocircle (6.3.4) about 0 onto the horocircle $\{z \in \mathbb{C} ; \operatorname{Im} z=1 / r-\operatorname{Re} \tau\}$ about $\infty$. The resulting group is not a terminal $b$-group of type $(1,1)$.

(6) It is easy to check that $C_{\tau+1}=A^{1 / 2} \circ C_{\tau}$, where $C_{\tau}$ is defined by (6.3.3). We do not know, however, whether $\tau+1 \in \mathbf{T}(1,1)$ whenever $\tau \in \mathbf{T}(1,1)$.

6.4. The surface $\Delta(\tau) / \Gamma_{2}(\tau)$ is obtained from $\mathbb{H}^{2} / F$ by a plumbing construction. Let $z$ and $w$ be the horocyclic coordinates on $\mathbb{H}^{2} / F$ at $\hat{\infty}$ with respect to $\hat{0}$ and at $\hat{0}$ with respect to $\hat{1}$. Then $z=e^{\pi i \zeta}$ and $w=e^{\pi i(1-1 / \zeta)}$, on an open subset $\Delta$ of $\mathbb{H}^{2}$. The HNN construction identifies a point $\zeta \in \Delta$ with $C(\zeta) \in \Delta$. Hence $z w=e^{\pi i C(\zeta)} e^{\pi i(1-1 / \zeta)}=e^{\pi i(\tau+1)}=t$. We conclude that $\Delta(\tau) / \Gamma_{2}(\tau)=S_{t}$ of $\S 2.4$

Remark. We can also compare local coordinates $z$ and $w$ at the puncture $\hat{\infty}$ coming from the groups $F$ and $C F C^{-1}$. The coordinate $z$ is, as above, the horocyclic coordinate on $\mathbb{H}^{2} / F$ at $\hat{\infty}$ with respect to $\hat{0}$; thus $z=e^{\pi i \zeta}$. The coordinate $w$ is the horocyclic coordinate on $\left(\mathbb{H}_{*}^{2}+i(\operatorname{Im} \tau)\right) / C F C^{-1}$ at $\hat{\infty}$ with respect to $\widehat{\tau+1}$; thus $w=e^{-\pi i(\zeta-\tau-1)}$. We obtain the same value of $t$, as expected.

Let $\Gamma$ now be an arbitrary torsion free terminal $b$-group of type $(1,1)$ with invariant component $\Delta$. Let $A \in \Gamma$ be a primitive accidental parabolic element of $\Gamma$. Choose the structure subgroup $F$ of $\Gamma$ that contains $A$. Choose $B^{-1} \in F$ that is conjugate to $A$ in $\Gamma$ (but not in $F$ ). Choose $C \in \Gamma$ that satisfies (6.3.1). Define a horocyclic coordinate $\tau$ and plumbing parameter $t$ by

$$
\tau=\tau(\Gamma)=-i \operatorname{tr} C, \quad t=t(\Gamma)=-e^{\pi i \tau(\Gamma)},
$$

where we choose a lift of $C$ to $\operatorname{SL}(2, \mathbb{C})$ with $\operatorname{Re}(\operatorname{tr} C)<0$.

Theorem. The plumbing parameter $t$ is a complete conjugacy class invariant for $\Gamma$. It satisfies $0<|t|<e^{-\pi}$ and $\Delta / \Gamma \cong S_{t}$. The horocyclic coordinate $\tau$ is determined modulo $2 \mathbb{Z}$ by $\Gamma$.

Proof. We have already seen that the only ambiguity is the choice of $C$ that satisfies (6.3.1). If $C$ and $\widetilde{C}$ both satisfy (6.3.1), then $\widetilde{C} \circ C^{-1} \in \Gamma$ and commutes with $A$. Hence it must be a power of $A$. Thus $\widetilde{C}=A^{m} \circ C$ for some $m \in \mathbb{Z}$ and this replaces $\tau$ by $\tau+2 m$. Hence $t$ is a conjugacy class invariant for $\Gamma$. The estimate on $|t|$ will be obtained in $\S 8.6$. In $\S 8.5$ we will show that $t$ is a complete invariant for the conjugacy class of $\Gamma$.

In analogy to the $(0,4)$ case, we write $F=F(a, b, c)$ with canonical generators $(A, B), a=f(A), b=f(B), c=A^{1 / 2}(b)$. Then we can express $\tau$ 
as a cross ratio

$$
\tau(\Gamma)=\operatorname{cr}(C(a), a, b, c),
$$

where $C(a)$ is the fixed point of the parabolic element $C \circ A \circ C^{-1}$ of $\Gamma$.

6.5. We need to study one more special case. Let $\tilde{\Gamma}$ be a terminal regular $b$ group of type $(p-1, n+2)$. Let $A_{1}$ and $A$ be parabolic elements of $\tilde{\Gamma}$ that determine distinct punctures on the surface represented by $\tilde{\Gamma}$ on its invariant component and that belong to different structure subgroups of $\tilde{\Gamma}$. Each of these elements is contained in a unique structure subgroup of $\tilde{\Gamma}$. Call these subgroups $F_{1}$ and $F$, respectively. Assume that $\Gamma$ is obtained from $\tilde{\Gamma}$ via an HNN-extension that conjugates $A_{1}$ onto $A$ by an element $C: C \circ A_{1} \circ C^{-1}=A$. Choose $B_{1}, B$ in $\tilde{\Gamma}$ so that $\left(A_{1}, B_{1}\right)$ and $(A, B)$ are canonical generators for $F_{1}$ and $F$. Then $\left(C \circ A_{1} \circ C^{-1}, C \circ B_{1} \circ C^{-1}\right)$ are canonical generators for $C F_{1} C^{-1}$. The group generated by $C F_{1} C^{-1}$ and $F$ is a terminal regular $b$ group of type $(0,4)$ : a modular subgroup of $\Gamma$. The element $C$ can be recovered from the horocyclic coordinate for this group. See $\S \S 7.5$ and 12.6.

\section{DEFORMATION SPACES FOR TORSION FREE TERMINAL $b$-GROUPS}

This section contains a description of the horocyclic coordinates on Teichmüller space determined by an admissible graph $\mathscr{G}$ as well as the construction of a regular terminal $b$-group $\Gamma_{t}$ for each $t \in \mathbf{D}_{0}(\mathscr{G})$.

7.1. Let $\mathscr{G}$ be an admissible graph of type $(p, n)$ with a semicanonical ordering for its edges. We adopt the notation and conventions introduced in $\S \S 3.2$ and 3.5. We will identify the edges and vertices of $\mathscr{G}$ with the partition curves and parts of the maximally partitioned surface $(S, \Sigma)$ associated with $\mathscr{G}$.

7.2. Let $\Gamma$ be a torsion free terminal $b$-group of graph type $\mathscr{G}$. We define horocyclic coordinates of $\Gamma$ by $\tau=\tau(\Gamma)=\left(\tau_{1}, \ldots, \tau_{d}\right)$, where, for $j=1, \ldots, d$, $\tau_{j}$ is a horocyclic coordinate of a modular subgroup $G_{j}$ of $\Gamma$ corresponding to the edge $a_{j}$ on $\mathscr{G}$ (see $\S \S 6.2$ and 6.4 ). The coordinates $\tau(\Gamma)$ are defined uniquely by the semicanonical ordering on the edges of $\mathscr{G}$ up to addition of a vector $\left(m_{1}, \ldots, m_{d}\right)$ in $\mathbb{Z}^{d} ; m_{j}$ is even whenever $a_{j}$ corresponds to an elliptic end on $\mathscr{G}$. Gluing or plumbing parameters for the group $\Gamma$ are defined by

$$
t=t(\Gamma)=\left(t_{1}, \ldots, t_{d}\right),
$$

where $t_{j}=-e^{\pi i \tau_{j}}$ if $a_{j}$ corresponds to an elliptic end on $\mathscr{G}$ and $t_{j}=e^{\pi i \tau_{j}}$ otherwise. If $\Delta$ is the invariant component of $\Gamma$, then $\Delta / \Gamma$ is conformally equivalent to $S_{t}$ (as a Riemann surface with a maximal partition) as a consequence of $\S \S 6.2,6.4$ and 3.4 .

We use $\mathbf{T}(\Gamma)$ as a model for $\mathbf{T}(p, n)$. Thus in our model, a point of $\mathbf{T}(p, n)$ is represented by an equivalence class of geometric isomorphisms of $\Gamma$ onto torsion free terminal $b$-groups of graph type $\mathscr{G}$. We shall say that the points in $\mathbf{T}(p, n)$ are marked groups of graph type $\mathscr{G}$. 
Theorem. Fix a graph $\mathscr{G}$ of type $(p, n)$. There exists a one-to-one holomorphic map $\tau: \mathbf{T}(p, n) \rightarrow \mathbb{C}^{d}$ (onto a bounded simply connected domain of holomorphy) with the property that for all $x \in \mathbf{T}(p, n), \tau(x)$ is a horocyclic coordinate for the marked group of graph type $\mathscr{G}$ (represented by $x$ ).

Proof. For $j=1,2, \ldots, d$, let us choose a primitive accidental parabolic element $A_{j} \in \Gamma$ that corresponds to the curve $a_{j} \in \Sigma$. Choose a modular subgroup $G_{j}$ of $\Gamma$ so that $A_{j}$ is accidental parabolic in $G_{j}$. Choose parabolic elements $B_{j 1}, B_{j 2}, B_{j 3}$ in $G_{j}$ so that a horocyclic coordinate for $G_{j}$ is given by $\operatorname{cr}\left(f\left(B_{j 3}\right), f\left(A_{j}\right), f\left(B_{j 1}\right), f\left(B_{j 2}\right)\right)$ as in $\S \S 6.2$ or 6.4. The map $\tau: \mathbf{T}(\Gamma) \rightarrow \mathbb{C}^{d}$ is defined by sending the geometric isomorphism $\theta: \Gamma \rightarrow \operatorname{PSL}(2, \mathbb{C})$ into the vector in $\mathbb{C}^{d}$ whose $j$ th component is

$$
\operatorname{cr}\left(f\left(\theta\left(B_{j 3}\right)\right), f\left(\theta\left(A_{j}\right)\right), f\left(\theta\left(B_{j 1}\right)\right), f\left(\theta\left(B_{j 2}\right)\right)\right) .
$$

We must prove that $\tau$ is holomorphic and injective. Holomorphicity of $\tau$ is a consequence of the holomorphic dependence on parameters of the solution of the Beltrami equation [AB]. The fact that $G_{j+1} \cap G_{j}$ is always a triangle group for $j=1, \ldots, d-1$ reduces the proof of injectivity to the one-dimensional case (see $[\mathrm{K} 6, \S 3])$. The one-dimensional situation was treated in $\S 6$.

Remark and definition. The above coordinates will be called horocyclic coordinates of graph type $\mathscr{G}$ for the Teichmüller space $\mathbf{T}(p, n)$. These are the coordinates described in our Introduction. We have begun the proof of Theorem 3 of the Introduction.

Remark. We can use an arbitrary ordering on the edges of $\mathscr{G}$ to determine horocyclic coordinates on $\mathbf{T}(p, n)$. These coordinates will differ from the ones arising from a semicanonical ordering on the edges, by a permutation and a translation (by a vector with integer entries).

7.3. We have shown that every torsion free terminal $b$-group $\Gamma$ of graph type $\mathscr{G}$ determines a Riemann surface $S_{t}, t \in \mathrm{D}_{0}(\mathscr{G})$. By varying the complex structure, using quasiconformal mappings (see, for example, Bers [B2, B3]), it follows that for all $t \in \mathbf{D}_{0}(\mathscr{G})$, the surface $S_{t}$ is so constructed. In $\S 7.5$ (see also §13) we produce an algorithm for obtaining $\Gamma$ from the gluing parameters $t \in \mathbf{D}_{0}(\mathscr{G})$. For the present we record the following

Theorem. Let $\Gamma$ be a torsion free terminal b-group of graph type $\mathscr{G}$. There exist surjective holomorphic mappings

$$
b: \mathbf{V}(\Gamma) \rightarrow \mathbf{V}_{0}(\mathscr{G}) \text { and } B: \mathbf{T}(\Gamma) \rightarrow \mathbf{D}_{0}(\mathscr{G})
$$

such that the following diagram commutes:

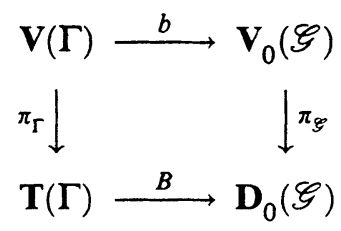


Proof. Using horocyclic coordinates $\tau$ for $\mathbf{T}(\Gamma)$, the map $B$ is defined by (7.2.1). Existence of $b$ is easily established once one shows that $\mathbf{V}_{0}(\mathscr{G})$ is a complex manifold (see the second paper of this series).

Remark. The above theorem shows that the constructions of the surfaces $S_{t}$, $t \in \mathbf{D}(\mathscr{G})$, given in $\S \S 3.4$ and 3.5, depend only on the graph $\mathscr{G}$ and the coordinates $t$.

7.4. The aim of this subsection is to describe formulae for the action of the Dehn twists about partition curves in terms of the horocyclic coordinates. Let $a$ be a simple closed curve on the Riemann surface $S$. Assume that $a$ is not contractible to either a point or a puncture on $S$. Then $\omega_{a}$, the left Dehn twist about the curve $a$, may be described as follows. Consider an annulus $\mathscr{A}$ around the curve $a$ (see Figure 9). Pick an orientation on $a$. Let $\mathscr{A}^{-}\left(\mathscr{A}^{+}\right)$ be the part of $\mathscr{A}$ lying to the left (right) of $a$. For $0 \leq \varphi \leq 2 \pi$, we let $\omega_{a, \varphi}$ be the left Dehn twist on $\mathscr{A}$ through the angle $\varphi$ about $a$. We use polar coordinates $(\rho, \theta)$ on $\mathscr{A}$ and normalize so that

$$
\begin{aligned}
\mathscr{A} & =\left\{z \in \mathbb{C} ; r^{-1}<\rho<r\right\}, \quad r>1, \\
a & =\{z \in \mathbb{C} ; \rho=1\},
\end{aligned}
$$

and

$$
\mathscr{A}^{-}=\left\{z \in \mathbb{C} ; r^{-1}<\rho<1\right\} \text {. }
$$

The formula for $\omega_{a, \varphi}$ is

$$
\omega_{a, \varphi}(\rho, \theta)= \begin{cases}(\rho, \theta), & r^{-1}<\rho \leq 1, \\ (\rho, \theta+\varphi(\rho-1) /(\sqrt{r}-1)), & 1 \leq \rho \leq \sqrt{r} \\ (\rho, \theta+\varphi), & \sqrt{r} \leq \rho<r .\end{cases}
$$

The Dehn twist $\omega_{a}$ is by definition $\omega_{a, 2 \pi}$ in $\mathscr{A}$ and the identity on $S-\mathscr{A}$. The isotopy class of the map $\omega_{a}$ is independent of the choice of orientation of the curve $a$.

We let $\omega_{k}$ be the left Dehn twist about the partition curve $a_{k}$. It is obvious that $\omega_{k}$ lifts to a quasiconformal automorphism of $\Delta$ that conjugates $\Gamma$ into itself. The lift (also denoted by $\omega_{k}$ ) is quasiconformal on $\hat{\mathbb{C}}$ (by an isomorphism theorem of Maskit [Mt4]) and reduces to a Möbius transformation on each noninvariant component of $\Gamma$ and on each structure region of $\Gamma$ (because the annulus $\mathscr{A}$ can be chosen to be arbitrarily small) that is disjoint from the structure loops corresponding to the partition curve $a_{k}$. To see this, let $D=\Delta-\pi^{-1}\left(\mathscr{A}_{k}\right)$, where $\mathscr{A}_{k}$ is a sufficiently small annulus around the curve $a_{k}$. For $z \in D, \omega_{k}(z)=\gamma(z)$, for some $\gamma \in \Gamma$ (that depends on $z$ ). By the discreteness of $\Gamma, \gamma$ is constant on each component of $D$. We conclude that $\omega_{k}$ conjugates each modular subgroup $G_{j}$ of $\Gamma$ into a $\Gamma$-equivalent subgroup. Without loss of generality, $\omega_{k} G_{k} \omega_{k}^{-1}=G_{k}$. Further, for each $j=1, \ldots, d$, $j \neq k$, there exists an $E_{k j} \in \Gamma$ such that $\omega_{k} \circ \gamma \circ \omega_{k}^{-1}=E_{k j} \circ \gamma \circ E_{k j}^{-1}$, all $\gamma \in G_{j}$. 


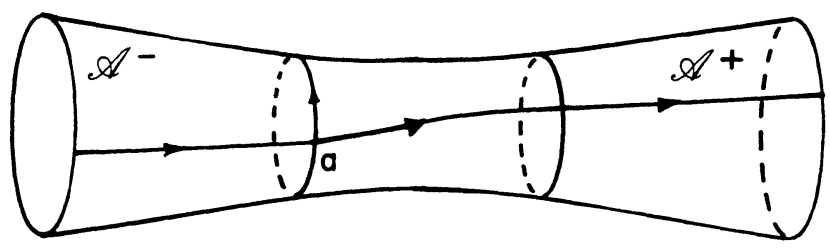

Partial Dehn twist about a.

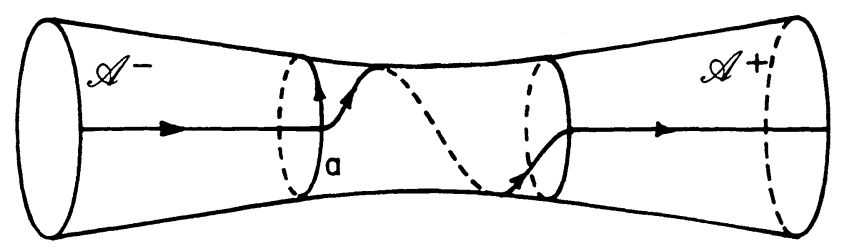

Full Dehn twist about a.

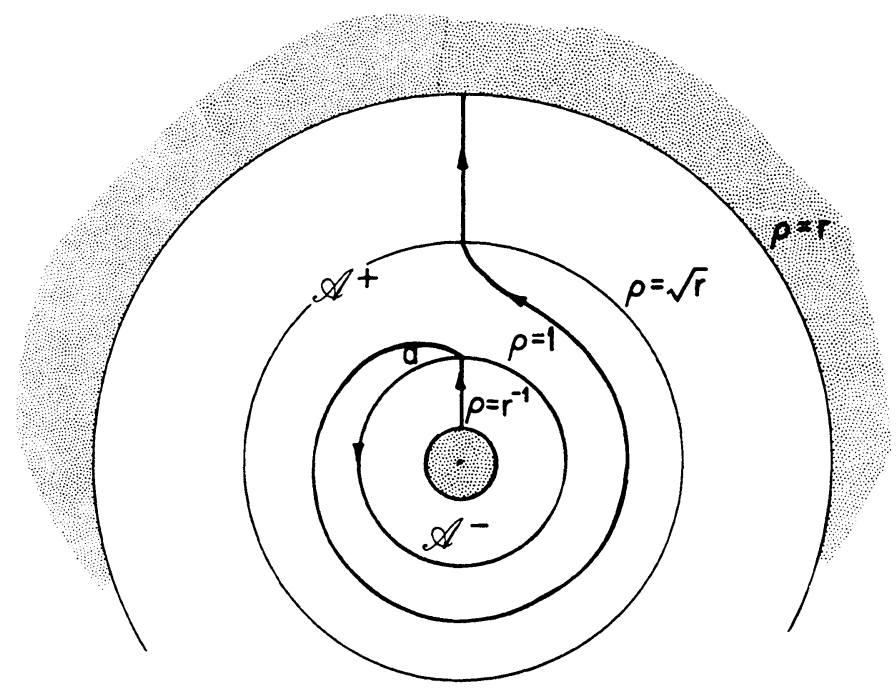

Alternate picture for full Dehn twist about a.

Figure 9. Dehn twist about a simple closed curve.

We use $\mathbf{T}(\Gamma)$ as the model for $\mathbf{T}(p, n)$ and study the action of $\omega_{k} \in N_{\mathrm{qc}}(\Gamma)$ on $\mathbf{T}(\Gamma)$. If $w$ is a $\Gamma$-compatible quasiconformal map $\left(\tilde{\Gamma}=w \Gamma w^{-1}\right)$, then $\tilde{\omega}_{k}=w \circ \omega_{k} \circ w^{-1}$ is a $\tilde{\Gamma}$-compatible quasiconformal map that restricts to an element of $\tilde{\Gamma}$ on each connected component of $w(D)$. It follows from (4.2.3) that for every geometric isomorphism $\theta$ of $\tilde{\Gamma}$, we have $\tau_{j}\left(\theta_{\omega_{k}}^{*}(\theta)\right)=\tau_{j}(\theta)$, $j=1, \ldots, d, j \neq k$.

To see what happens to $\tau_{k}$, we may assume that $k=1$ and that $d=1$. Assume that $\Gamma=\mathscr{G}_{1}$ is of type $(0,4)$ and use the notation of $\S 6.1$ (recall Figure 7). We may assume that the curve $a=a_{1}$ corresponds to the Möbius transformation $A=\left[\begin{array}{cc}-1 & -2 \\ 0 & -1\end{array}\right]$, and that, without loss of generality, $\operatorname{Im} \alpha>1$. The 
annulus $\mathscr{A}$ is the image of the region described in (6.2.1) under the canonical projection $\zeta \mapsto z=e^{\pi i(\zeta-\alpha / 2)}$. The polar coordinates $(\rho, \theta)$ on $\mathscr{A}$ are hence given by

$$
\rho=|z|=e^{-\pi \operatorname{Im}(\zeta-\alpha / 2)}, \quad \theta=\pi \operatorname{Re}\left(\zeta-\frac{1}{2} \alpha\right) .
$$

The curve $a$ is represented by $\rho=1$; that is, by $\left\{\zeta \in \mathbb{C} ; \zeta=x+\frac{i}{2} \operatorname{Im} \alpha\right.$, $0 \leq x \leq 2\}$. The parameter $r$ of the annulus $\mathscr{A}$ is given by $r=e^{\pi(\operatorname{Im} \alpha-1) / 2}$; the region in (6.2.1) where $\operatorname{Im} \zeta>\frac{1}{2} \operatorname{Im} \alpha$ is projected onto $\mathscr{A}^{-}$. Let $\omega$ be a lift of the Dehn twist about $a$. To specify $\omega$ uniquely, we require that $\omega$ be the identity on the lift (under $z$ ) of $\mathscr{A}^{-}$; that is, on $\left\{\zeta \in \mathbb{C} ; \frac{1}{2} \operatorname{Im} \alpha<\operatorname{Im} \zeta<\right.$ $\left.\operatorname{Im} \alpha-\frac{1}{2}\right\}$. It follows that

$$
\begin{aligned}
& \omega(\zeta)=\zeta, \quad \zeta \in \Delta, \frac{1}{2} \operatorname{Im} \alpha \leq \operatorname{Im} \zeta, \\
& \omega(\zeta)=\zeta+4 \frac{\operatorname{Im}(\alpha-2 \zeta)}{\operatorname{Im} \alpha-1}, \quad \zeta \in \Delta, \frac{1}{4}(\operatorname{Im} \alpha+1) \leq \operatorname{Im} \zeta \leq \frac{1}{2} \operatorname{Im} \alpha,
\end{aligned}
$$

and

$$
\omega(\zeta)=\zeta+2, \quad \zeta \in \Delta, \operatorname{Im} \zeta \leq \frac{1}{4}(\operatorname{Im} \alpha+1) .
$$

Using the notation and results of Remark (5) of $\S 6.1$ and of $\S 4.4$, we see that if $w$ is a normalized $\Gamma$-compatible quasiconformal automorphism of $\hat{\mathbb{C}}$, then

$$
\begin{aligned}
& \operatorname{cr}\left(w \circ \omega^{-1}\left(\alpha_{0}\right), w \circ \omega^{-1}(\infty), w \circ \omega^{-1}(0), w \circ \omega^{-1}(1)\right) \\
& \quad=\operatorname{cr}\left(w\left(\alpha_{0}\right), w(\infty), w(-2), w(-1)\right)=\operatorname{cr}(\alpha, \infty,-2,-1)=\alpha+2 .
\end{aligned}
$$

The next to the last equality follows from the observation that a normalized $\Gamma$-compatible quasiconformal map commutes with each element of $F_{1}=$ $F(\infty, 0,1)$ and hence must be the identity on $\Lambda\left(F_{1}\right)=\hat{\mathbb{R}}$. We have established the following

Theorem. In the horocyclic coordinates described by Theorem 7.2, the action of the Dehn twist around $a_{k}, k=1,2, \ldots, d$, is given by

$$
\tau_{j} \mapsto \tau_{j} \text { for } j=1, \ldots, d, j \neq k \text {, and } \tau_{k} \mapsto \tau_{k}+2 .
$$

Remark. We leave it to the reader to perform the appropriate analysis for the case where the modular subgroup $G_{k}$ is of type $(1,1)$. We have proven part (b) of Theorem 3 of the Introduction.

To describe the geometric automorphism $\theta: \Gamma \rightarrow \Gamma$ induced by a Dehn twist, we consider a simple closed curve $a$ on $S$ that is represented by an accidental parabolic element $A \in \Gamma$. Let $\tilde{a}$ be a structure loop in $\Delta$, precisely invariant under $\langle A\rangle$ in $\Gamma$, with $\pi(\tilde{a})=a$. Let us orient $\tilde{a}$ so that for all $z_{0} \in \tilde{a}$, the points $A^{-1}\left(z_{0}\right), z_{0}$ and $A\left(z_{0}\right)$ follow each other in the positive orientation. We consider the two cases:

(I) $S-a$ consists of two components $S_{1}$ and $S_{2}$. Assume that $S_{1}$ lies to the right of $a$. We choose connected components $\Delta_{i}$ of $\pi^{-1}\left(S_{i}\right), i=1,2$, with the property that the closures of $\Delta_{1}$ and $\Delta_{2}$ (in $\Delta$ ) intersect in $\tilde{a}$. Let $\Gamma_{i}$ be 
the stabilizer of $\Delta_{i}$ in $\Gamma$. Then $\Gamma=\Gamma_{1} *_{\langle A\rangle} \Gamma_{2}$. We choose a lift $\omega$ of $\omega_{a}$ that is the identity on $\Delta_{1}$. Then

$$
\theta(\gamma)=\omega^{-1} \circ \gamma \circ \omega= \begin{cases}\gamma, & \gamma \in \Gamma_{1}, \\ A \circ \gamma \circ A^{-1}, & \gamma \in \Gamma_{2} .\end{cases}
$$

(II) $S-a=S_{1}$ is connected. Let $\Delta_{1}$ be a connected component of $\pi^{-1}\left(S_{1}\right)$ with $\tilde{a}$ on the boundary of $\Delta_{1}$ and $\Delta_{1}$ lying to the right of $\tilde{a}$. Let $\Gamma_{1}$ be the stabilizer of $\Delta_{1}$ in $\Delta$. Then $\Gamma$ is an HNN-extension of $\Gamma_{1}$ by an element $C \in \Gamma-\Gamma_{1}$ that conjugates $A \in \Gamma_{1}$ to $C^{-1} \circ A \circ C \in \Gamma_{1}$. The lift $\omega$ of $\omega_{a}$ that is the identity on $\Delta_{1}$ satisfies

$$
\theta(\gamma)=\omega^{-1} \circ \gamma \circ \omega= \begin{cases}\gamma, & \gamma \in \Gamma_{1}, \\ A \circ \gamma, & \gamma=C .\end{cases}
$$

7.5. In this subsection we prove Theorem 2. Let $t \in \mathbf{D}_{0}(\mathscr{G})$ and choose $\tau_{j} \in \mathbb{C}^{*}$ such that $t_{j}=-e^{\pi i \tau_{j}}$ if the edge $a_{j}$ on $\mathscr{G}$ represents an elliptic end and $t_{j}=e^{\pi i \tau_{j}}$ otherwise. We describe an algorithm ${ }^{8}$ for producing a unique group $\Gamma_{t}$ of graph type $\mathscr{G}$ that represents the surface $S_{t}$. The algorithm produces the generators and relations for $\Gamma_{t}$ and keeps track of the conjugacy classes of parabolic elements corresponding to the edges of $\mathscr{G}$ (these are the accidental parabolic elements) as well as to the phantom edges of $\mathscr{G}$ (these are the parabolic elements that correspond to punctures on $S_{t}$ ). Thus the algorithm also produces (automatically) representatives for the structure subgroups and modular subgroups of $\Gamma$. In listing parabolic elements corresponding to punctures, we follow the convention that punctures lie to the left of the corresponding oriented horocircles.

For $k=0,1, \ldots, d$, let $t^{k}$ be the vector in $\mathbb{C}^{k}$ obtained by ignoring the last $d-k$ coordinates of $t\left(t^{0}\right.$ is by convention 0$)$.

We represent the vertex $S^{1}$ of $\mathscr{G}$ by the group $F_{1}=F=F(\infty, 0,1)$ of $\S 1.2$. We assign the parabolic elements $A, B, B^{-1} A^{-1}$ to the punctures $P^{11}, P^{12}, P^{13}$ on $S^{1}$ (we use the action of $F$ on $\mathbb{H}^{2}$ ). The group $F=\Gamma_{0}$ represents the thrice punctured sphere $S_{0}$.

Let $k=0,1, \ldots, d-1$. Having constructed the group $\Gamma_{t^{k}}=\tilde{\Gamma}$ (with invariant component $\tilde{\Delta}$ ) of graph type $\mathscr{G}_{k}$ that represents the surface $S_{t^{k}}$, we proceed to construct the group $\Gamma_{t^{k+1}}=\Gamma$ of graph type $\mathscr{G}_{k+1}$ that represents the surface $S_{t^{k+1}}$. We assume that we have assigned to each edge (including the phantom edges) of $\mathscr{G}_{k}$ a parabolic element of $\Gamma_{t^{k}}$ that represents the corresponding partition curve (or puncture) on $S_{t^{k}}$. We now assume that the edge

\footnotetext{
${ }^{8}$ It is clear from $\S 7.2$ that such an algorithm should exist. The purpose of this subsection is to show that this algorithm is rational in $\log t_{j}$. The algorithm produces a group $\Gamma_{t}$ for each $t \in \mathbb{C}^{d}$ with $|t|<1$ and all components of $t$ nonzero (see also §13.) The group is a torsion free terminal $b$-group if and only if $t \in \mathbf{D}_{0}(\mathscr{G})$.
} 
$a_{k+1} \in \mathscr{G}_{k+1}-\mathscr{G}_{k}$ originates at the puncture $P^{j i}$ and terminates at the puncture $P^{j^{\prime} i^{\prime}}$. We conisder the three possible cases:

(I) The edge $a_{k+1}$ disconnects $\mathscr{G}_{k+1}$. In this case $j^{\prime} \neq j, S^{j} \in \mathscr{G}_{k}$ and $S^{j^{\prime}} \in$ $\mathscr{G}_{k+1}-\mathscr{G}_{k}$. We start with the parabolic element $A_{1} \in \tilde{\Gamma}$ that has been assigned to the puncture $P^{j i}$. Choose the structure subgroup $F_{1}$ of $\tilde{\Gamma}$ that contains the parabolic motion $A_{1}$. Let $B_{1}$ be the parabolic element of $F_{1}$ that has been assigned to the puncture $P^{j i+1}$. Then (by induction) $\left(A_{1}, B_{1}\right)$ are canonical generators for $F_{1}$. Solve for $\tilde{b}_{2}$ in

$$
\tau_{k+1}=\operatorname{cr}\left(\tilde{b}_{2}, f\left(A_{1}\right), f\left(B_{1}\right), f\left(B_{1}^{-1} \circ A_{1}^{-1}\right)\right) .
$$

Use Lemma 12.2 with $A=A_{1}^{-1}$ and $b=\tilde{b}_{2}$ to solve for $B=B_{2}$. The group $\Gamma$ is $\tilde{\Gamma}$ with $B_{2}$ adjoined.

We assign the parabolic elements $B_{2}$ to the puncture $P^{j^{\prime} i^{\prime}+1}$. The parabolic element $B_{2}^{-1} \circ A_{1}$ is automatically assigned to the puncture $P^{j^{\prime} i^{\prime}+2}$. The new structure subgroup (for the vertex $S^{j^{\prime}}$ ) is the triangle group

$$
F_{2}=F\left(f\left(A_{1}^{-1}\right), f\left(B_{2}\right), f\left(B_{2}^{-1} \circ A_{1}\right)\right) \text {. }
$$

The new modular subgroup (for the edge $a_{k+1}$ ) is the amalgamated free product (AFP) of $F_{1}=F\left(f\left(A_{1}\right), f\left(B_{1}\right), f\left(B_{1}^{-1} \circ A_{1}\right)\right)$ and $F_{2}$ across the cyclic parabolic subgroup $\left\langle A_{1}\right\rangle$.

(II) The edge $a_{k+1}$ joins two distinct vertices on $\mathscr{G}_{k+1}$ but does not disconnect $\mathscr{G}_{k+1}$. In this case $j^{\prime} \neq j$ but $S^{j}$ and $S^{j^{\prime}} \in \mathscr{G}_{k}$. Start with the parabolic elements $A_{1}$ and $A_{2}$ of $\tilde{\Gamma}$ that have been assigned to the punctures $P^{j i}$ and $P^{j^{\prime} i^{\prime}}$. Choose the structure subgroup $F_{2}\left(F_{1}\right)$ of $\tilde{\Gamma}$ that contains the parabolic motion $A_{2}\left(A_{1}\right)$. Let $B_{1} \in F_{1} \quad\left(B_{2} \in F_{2}\right)$ be the parabolic motion that has been assigned to the puncture $P^{j i+1}\left(P^{j^{\prime} i^{\prime}+1}\right)$. Then $\left(A_{j}, B_{j}\right)$ are canonical generators for $F_{j}, j=1,2$. Solve for $\tilde{b}_{2}$ in (7.5.1) with $A=A_{1}$. Use Lemma 12.2 with $A=A_{1}$ and $b=\tilde{b}_{2}$ to solve for $B=\tilde{B}_{2}$. Then $\left(A_{1}, B_{1}\right)$ and $\left(A_{1}, \tilde{B}_{2}\right)$ are canonical generators for distinct triangle groups $F_{1}$ and $\tilde{F}$. Use the Remark in $\S 1.3$ or Proposition 12.6 to solve for $C$, where

$$
C \circ A_{2} \circ C^{-1}=A_{1}, \quad C \circ B_{2} \circ C^{-1}=\tilde{B}_{2}, \quad C F_{2} C^{-1}=\tilde{F} .
$$

The group $\Gamma$ is $\tilde{\Gamma}$ with $C$ adjoined. The modular subgroup corresponding to the edge $a_{k+1}$ is the AFP of $F_{1}$ and $\tilde{F}$ across $\left\langle A_{1}\right\rangle$.

(III) The edge $a_{k+1}$ joins the vertex $S^{j}$ on $\mathscr{G}_{k+1}$ to itself. In this case $j^{\prime}=j$ and the edge $a_{k+1}$ starts at the puncture $P^{j i}$ and ends at $P^{j i+1}$. Let $A$ and $B$ be the parabolic elements assigned to the punctures $P^{j i}$ and $P^{j i+1}$. Then $(A, B)$ are canonical generators for the structure subgroup $F$ of $\tilde{\Gamma}$ corresponding to 

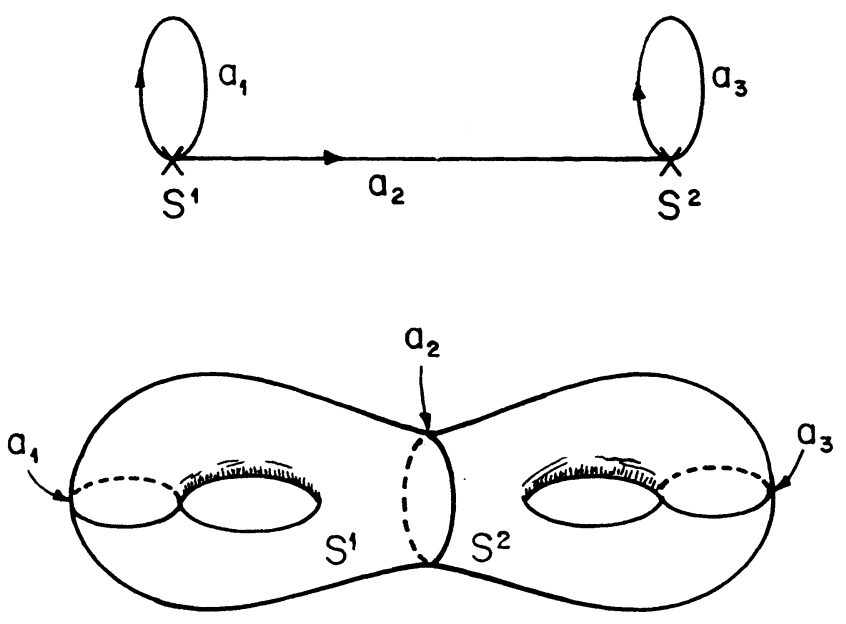

FIgure 10. A trivalent graph and corresponding maximal partition for a surface of genus 2 .

the vertex $S^{j}$. Solve for $C$ using Proposition 12.4 with $\tau=\tau_{k+1}$. The group $\Gamma$ is then $\tilde{\Gamma}$ with $C$ adjoined. The modular group corresponding to the edge $a_{k+1}$ is the HNN-extension of $F$ by the motion $C$.

Remarks. (1) As a result of $\S 12.5$, construction (III) can be considered a special case of (II).

(2) The parabolic elements assigned to the punctures $P^{j i}$ depend on the horocyclic coordinates of the group $\Gamma_{t}$ (that is, they depend on the choice of the logarithms of the $t_{k}$ 's). However, the group $\Gamma_{t}$ is independent of these choices; it depends only on the graph $\mathscr{G}$ (with its semicanonical ordering) and the plumbing parameters $t$.

Two illustrative examples. There are exactly two combinatorially distinct maximal partitions of a compact surface of genus 2; these are illustrated in Figures 10 and 11 (see p. 548).

The Figure 10 construction. Let $\Gamma_{k}$ be the group corresponding to the graph $\mathscr{G}_{k}$. (Thus $\Gamma=\Gamma_{3}, \mathscr{G}=\mathscr{G}_{3}$.) The vertex $S^{1}$ has boundary components $P^{11}, P^{12}, P^{13}$, while $S^{2}$ has boundary components $P^{21}, P^{22}, P^{23}$. We view (see $\S 3.2$ ) the edge $a_{1}$ as originating at $P^{11}$ and ending at $P^{12}$; the edge $a_{2}$ starts at $P^{13}$ and ends at $P^{21}$; the edge $a_{3}$ starts at $P^{22}$ and ends at $P^{23}$. We start with the group $F$ of $\S 1.2$ that represents the part $S^{1}$ (see also $\S 12.1$ ): $F_{1}=F(\infty, 0,1)$, with canonical generators $(A, B)$ representing the punctures $P^{11}$ and $P^{12}$, respectively. 

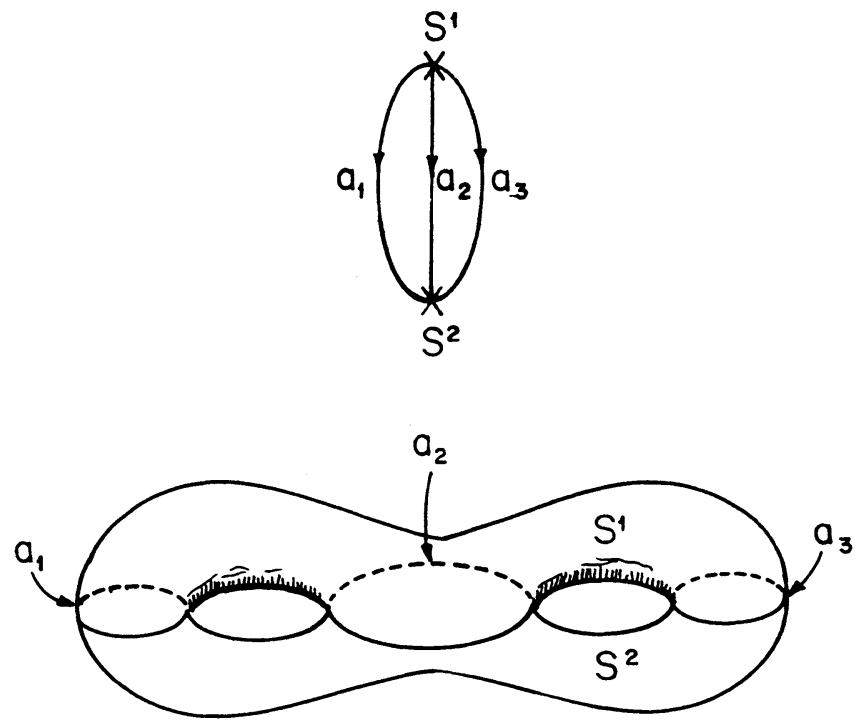

FIGURE 11. The second trivalent graph on two vertices.

The construction corresponding to $a_{1}$ is of type (III). The group $\Gamma_{1}$ is described by

$$
\begin{aligned}
\Gamma_{1}=\left\langle A, C_{1} ; A\right. & \text { is accidental parabolic, }\left(B^{-1} \circ A^{-1}\right) \\
& \left.=C_{1}^{-1} \circ A \circ C_{1} \circ A^{-1}=\left[C_{1}, A^{-1}\right] \text { is parabolic }\right\rangle .
\end{aligned}
$$

The motion $C_{1}$ is uniquely defined (see Proposition 12.4) by the conditions $C_{1} \circ B^{-1} \circ C_{1}^{-1}=A$ and $\operatorname{cr}\left(C_{1}(f(A)), f(A), f(B), f\left(A^{-1} \circ B^{-1}\right)\right)=\tau_{1}$. Thus (see also §6.3) $C_{1}=i\left[\begin{array}{cc}\tau_{1} & 1 \\ 1 & 0\end{array}\right]$ and $\Gamma_{1}=G_{1}$ is the modular subgroup corresponding to the partition curve $a_{1}$. For notational convenience, we relabel $A=A_{1}$.

The construction corresponding to the edge $a_{2}$ is of type (I). We start with the group $F_{1}=F(1, \infty, 0)$ with canonical generators $\left(B^{-1} \circ A_{1}^{-1}, A_{1}\right)$ determined from the cyclic ordering of the boundary components of $S^{1}$. We adjoin an element $B_{2}$ (corresponding to the puncture $P^{22}$ ) according to Lemma 12.2; that is, we solve for $f\left(B_{2}\right)=\alpha_{2}$ in $\tau_{2}=\operatorname{cr}\left(\alpha_{2}, 1, \infty, 0\right)$ (thus $\alpha_{2}=1-1 / \tau_{2}$ ), and construct a new triangle group $F_{2}$ with canonical generators $\left(A_{1} \circ B, B_{2}\right)$. We have:

$$
\begin{gathered}
F_{2}=F\left(1,1-\frac{1}{\tau_{2}}, \frac{\tau_{2}-2}{\tau_{2}-1}\right), \\
B_{2}=\left[\begin{array}{cc}
-1+2 \tau_{2}\left(1-\tau_{2}\right) & 2\left(1-\tau_{2}\right)^{2} \\
-2 \tau_{2}^{2} & -1-2 \tau_{2}\left(1-\tau_{2}\right)
\end{array}\right] .
\end{gathered}
$$

See Lemma 12.2 and its Corollary 1. The modular subgroup $G_{2}$ corresponding 
to the curve $a_{2}$ is the free group generated by $B^{-1} \circ A_{1}^{-1}, A_{1}$ and $B_{2}:$ the AFP of $F_{1}$ and $F_{2}$ across $\left\langle B^{-1} \circ A_{1}^{-1}\right\rangle$. We relabel $B^{-1} \circ A_{1}^{-1}=A_{2}$. The presentation for $\Gamma_{2}$ (a group of type $(1,2)$ ) is

$$
\begin{gathered}
\Gamma_{2}=\left\langle A_{1}, C_{1}, B_{2} ; A_{1} \text { and } A_{2}=\left[C_{1}, A_{1}^{-1}\right]\right. \text { are accidental parabolic, } \\
\left.B_{2} \text { and } B_{2}^{-1} \circ A_{2} \text { are parabolic, }\left[A_{1}^{-1}, C_{1}\right] \circ B_{2} \circ\left(B_{2}^{-1} \circ A_{2}\right)=I\right\rangle .
\end{gathered}
$$

(The last equality may be omitted.)

The construction corresponding to the edge $a_{3}$ is of type (III). We start with the group $F_{2}=F\left(1-1 / \tau_{2},\left(\tau_{2}-2\right) /\left(\tau_{2}-1\right), 1\right)$ with canonical generators $\left(B_{2}, B_{2}^{-1} \circ A_{2}\right)$. We adjoin the unique Möbius transformation $C_{3}$ with (see §12.4) $C_{3} \circ\left(B_{2}^{-1} \circ A_{2}\right)^{-1} \circ C_{3}^{-1}=B_{2}$ and

$$
\operatorname{cr}\left(C_{3}\left(f\left(B_{2}\right)\right), f\left(B_{2}\right), f\left(B_{2}^{-1} \circ A_{2}\right), f\left(A_{2}^{-1}\right)\right)=\tau_{3} ;
$$

it is given by

$$
C_{3}=i\left[\begin{array}{ll}
\tau_{3} \tau_{2}^{2}+2\left(1-\tau_{3}\right) \tau_{2}+\tau_{3}-2 & -\tau_{3} \tau_{2}^{2}+\left(3 \tau_{3}-2\right) \tau_{2}-2 \tau_{3}+3 \\
\tau_{3} \tau_{2}^{2}+\left(2-\tau_{3}\right) \tau_{2}-1 & -\tau_{3} \tau_{2}^{2}-2\left(1-\tau_{3}\right) \tau_{2}+2
\end{array}\right] .
$$

The modular subgroup $G_{3}$ corresponding to $a_{3}$ is generated freely as an abstract group by the Möbius transformations $B_{2}$ and $C_{3}$. We relabel $B_{2}^{-1}=$ $A_{3}$. The presentation for $\Gamma=\Gamma_{3}$ is

$$
\begin{array}{r}
\Gamma=\left\langle A_{1}, C_{1}, A_{3}, C_{3} ; A_{1}, A_{2}=\left[C_{1}, A_{1}^{-1}\right] \text { and } A_{3}\right. \text { are accidental parabolic, } \\
\left.\left[A_{1}^{-1}, C_{1}\right] \circ\left[A_{3}, C_{3}\right]=I\right\rangle .
\end{array}
$$

The Figure 11 construction. We view $a_{j}$ as joining the puncture $P^{1 j}$ on $S^{1}$ to $P^{2 j}$ on $S^{2}, j=1,2,3$. We start once again with $F_{1}=F(\infty, 0,1)$ and canonical generators $(A, B)$ representing the punctures $P^{11}, P^{12}$. The group $\Gamma_{1}=G_{1}$ is the AFP of $F_{1}$ with $F_{2}=F\left(\infty, \tau_{1}, \tau_{1}-1\right)$ across $\langle A\rangle$; the canorical generators for $F_{2}$ are $\left(A^{-1}, B_{1}=B_{\tau_{1}}^{-1}\right)$ with $B_{1}$ representing the puncture $P^{22}$. We relabel $A=A_{1}$. The standard presentation for $\Gamma_{1}$ is

$$
\begin{aligned}
\Gamma_{1}=\left\langle A_{1}, B, B_{1} ; A_{1}\right. & \text { is accidental parabolic, } B, B^{-1} \circ A_{1}^{-1}, B_{1}, B_{1}^{-1} \circ A_{1} \\
& \text { are parabolic, } \left.B \circ\left(B^{-1} \circ A_{1}^{-1}\right) \circ B_{1} \circ\left(B_{1}^{-1} \circ A_{1}\right)=I\right\rangle .
\end{aligned}
$$

The construction corresponding to the edge $a_{1}$ was of type (I). We proceed to the construction of type (II) corresponding to the edge $a_{2}$. We will adjoin an element $C_{2}$ that satisfies $C_{2} \circ B^{-1} \circ C_{2}^{-1}=B_{1}$. We first construct $G_{2}$; it is the AFP of $F_{1}=F(0,1, \infty)$ with $F\left(0, b_{2}, B^{-1 / 2}\left(b_{2}\right)\right)$, with canonical generators $\left(B^{-1}, B_{2}\right)$, across $\langle B\rangle$. Here

$$
\tau_{2}=\operatorname{cr}\left(b_{2}, 0,1, \infty\right), \quad b_{2}=\frac{1}{1-\tau_{2}} \quad \text { and } \quad B^{-1 / 2}\left(b_{2}\right)=\frac{1}{2-\tau_{2}} .
$$


Now $F\left(0, b_{2}, B^{-1 / 2}\left(b_{2}\right)\right)$ is conjugate via $C_{2}$ to $F\left(\tau_{1}, \tau_{1}-1, \infty\right)$; that is,

$$
C_{2} F\left(0, b_{2}, B^{-1 / 2}\left(b_{2}\right)\right) C_{2}^{-1}=F\left(\tau_{1}, \tau_{1}-1, \infty\right) \text {. }
$$

Thus $C_{2}$ conjugates $B^{-1}$ to $B_{1}$ and is given by

$$
C_{2}=\left[\begin{array}{cc}
\tau_{1}\left(\tau_{2}-2\right)+1 & \tau_{1} \\
\tau_{2}-2 & 1
\end{array}\right]
$$

The standard presentation for $\Gamma_{2}$ is $\left(B=A_{2}\right)$

$\Gamma_{2}=\left\langle A_{1}, A_{2}, C_{2} ; A_{1}, A_{2}\right.$ are accidental parabolic, $A_{2}^{-2} \circ A_{1}^{-1}, C_{2} \circ A_{2} C_{2}^{-1} \circ A_{1}$ are parabolic, $\left.\left[C_{2}^{-1}, A_{2}\right] \circ\left(A_{2}^{-1} \circ A_{1}^{-1}\right) \circ\left(A_{1} \circ C_{2} \circ A_{2} \circ C_{2}^{-1}\right)=I\right\rangle$.

The construction corresponding to the edge $a_{3}$ is also of type (II). The modular subgroup $G_{3}$ is the AFP of $F_{1}=F(1, \infty, 0)$ with $F\left(1, b_{3},(A \circ B)^{1 / 2}\left(b_{3}\right)\right)$ across $\left\langle B^{-1} \circ A^{-1}\right\rangle$. Here

$$
\tau_{3}=\operatorname{cr}\left(b_{3}, 1, \infty, 0\right), \quad b_{3}=\frac{\tau_{3}-1}{\tau_{3}} \text { and }(A \circ B)^{1 / 2}\left(b_{3}\right)=\frac{\tau_{3}-2}{\tau_{3}-1} .
$$

The new group $F\left(1, b_{3},(A \circ B)^{1 / 2}\left(b_{3}\right)\right)$ is conjugate via $C_{3}$ to $F\left(\tau_{1}-1, \infty, \tau_{1}\right)$; that is,

$$
C_{3} F\left(1, b_{3},(A \circ B)^{1 / 2}\left(b_{3}\right)\right) C_{3}^{-1}=F\left(\tau_{1}-1, \infty, \tau_{1}\right) .
$$

Thus $C_{3} \circ(A \circ B) \circ C_{3}^{-1}=B_{1}^{-1} \circ A$, and

$$
C_{3}=\left[\begin{array}{cc}
\left(\tau_{1}-1\right) \tau_{3}+1 & \left(1-\tau_{1}\right) \tau_{3}+\tau_{1}-2 \\
\tau_{3} & 1-\tau_{3}
\end{array}\right]
$$

The standard presentation for $\Gamma=\Gamma_{3}$ is

$$
\begin{array}{r}
\Gamma=\left\langle A_{1}, A_{2}, C_{2}, C_{3} ; A_{1}, A_{2} \text { and } A_{3}=A_{1} \circ A_{2}\right. \text { are accidental parabolic, } \\
\left.\left[C_{2}^{-1}, A_{2}\right] \circ\left[A_{1} \circ A_{2}, C_{3}^{-1} \circ A_{1}^{-1}\right]=I\right\rangle .
\end{array}
$$

The group $\Gamma$ is constructed from $F_{1}$ and $F_{2}$ by one AFP and two HNNextensions.

7.6. We extend the constructions of $\S 7.5$ to include the case where $t \in \mathbf{D}(\mathscr{G})$ $\mathbf{D}_{0}(\mathscr{G})$. Let $\mathscr{G}^{\prime}$ be the augmented subgraph of $\mathscr{G}$ obtained by breaking each edge $a_{j}$ for which $t_{j}=0$. Let $r$ be the number of nodes on $S_{t}$ (equivalently, the number of zero components of the vector $t$ ). Let $\mathscr{G}_{1}, \ldots, \mathscr{G}_{s}$ be the connected components of $\mathscr{G}^{\prime}$ and let $t^{k}, k=1, \ldots, s$, be the vector containing only the components of $t$ corresponding to the edges in $\mathscr{G}_{k}$. Then $t^{k} \in \mathbf{D}_{0}\left(\mathscr{G}_{k}\right)$ and the singular surface $S_{t}$ is obtained from the disjoint union $S^{0}=S_{t^{1}} \cup \cdots \cup S_{t^{s}}$ by identifying $r$ distinct pairs of punctures to form nodes. Let $a_{1}, b_{1}, \ldots, a_{r}, b_{r}$ be the list of phantom edges on $\mathscr{G}^{\prime}$ so that $\mathscr{G}$ is constructed from $\mathscr{G}^{\prime}$ by connecting each of the $r$ pairs of phantom edges $a_{j}, b_{j}$ to form an edge. 
We reorder the graphs $\mathscr{G}_{1}, \ldots, \mathscr{G}_{s}$ and the phantom edges $a_{1}, b_{1}, \ldots, a_{r}$, $b_{r}$, if necessary, so that for $j=1, \ldots, s-1, a_{j}$ is a phantom edge on the graph $\mathscr{G}_{j+1}$ and $b_{j}$ is a phantom edge on some $\mathscr{G}_{k}$ with $k=k(j) \leq j$. Each of the $2 r$ phantom edges determines a puncture on the surface $S^{0}$.

A configuration $\Gamma$ of graph type $\mathscr{G}$ representing the surface $S_{t}$ is a collection

$$
\Gamma=\left\{\Gamma_{1}, \ldots, \Gamma_{s} ; A_{1}, \ldots, A_{s-1} ; R_{s}, \ldots, R_{r}\right\},
$$

where:

(i) For $j=1, \ldots, s, \Gamma_{j}$ is a terminal torsion free $b$-group of graph type $\mathscr{G}_{j}$ that represents the surface $S_{t^{j}}$. We call $\Gamma_{j}$ a component group of the configuration $\Gamma$.

(ii) For $j=1, \ldots, s-1$, the punctures $a_{j}$ on $S_{t^{j+1}}$ and $b_{j}$ on $S_{t^{k(j)}}$ are represented by the parabolic element $A_{j} \in \Gamma_{j+1} \cap \Gamma_{k(j)}$. Further, the puncture $a_{j}$ lies to the left (right) of the horocircles determined by $A_{j}$ if and only if the puncture $b_{j}$ lies to the right (left) of the horocircles.

(iii) For $j=s, \ldots, r, R_{j}$ is an identification of the conjugacy class $\left\{\left\{A_{j}\right\}\right\}$ of parabolic elements in $\Gamma_{k(j)}$ representing the puncture $a_{j}$ on $S_{t^{k(j)}}$ with the conjugacy class $\left\{\left\{B_{j}\right\}\right\}$ of parabolic elements in $\Gamma_{m(j)}$ representing the puncture $b_{j}$ on $S_{t^{m(j)}}$. Further, as in (ii), the horocircles determined by $A_{j}$ lie to the left (right) of the puncture $a_{j}$ if and only if the horocircles determined by $B_{j}$ lie to the right (left) of the puncture $b_{j}$.

Two configurations (7.6.1) and

$$
\Gamma^{\prime}=\left\{\Gamma_{1}^{\prime}, \ldots, \Gamma_{s}^{\prime} ; A_{1}^{\prime}, \ldots, A_{s-1}^{\prime} ; R_{s}^{\prime}, \ldots, R_{r}^{\prime}\right\}
$$

are conjugate if there exist Möbius transformations $C_{1}, \ldots, C_{s}$ so that

$$
\Gamma_{j}^{\prime}=C_{j} \Gamma_{j} C_{j}^{-1}, \quad j=1, \ldots, s,
$$

and the resulting (not necessarily well-defined) map $\theta: \Gamma_{1} \cup \cdots \cup \Gamma_{s} \rightarrow \Gamma_{1}^{\prime} \cup \cdots \cup \Gamma_{s}^{\prime}$ satisfies

$$
\theta\left(A_{j}\right)=A_{j}^{\prime} \text { for } j=1, \ldots, s-1
$$

and

$$
\theta\left(R_{j}\right)=R_{j}^{\prime} \text { for } j=s, \ldots, r
$$

Condition (7.6.2) means that $A_{j}^{\prime}=C_{j} \circ A_{j} \circ C_{j}^{-1}=C_{k(j)} \circ A_{j} \circ C_{k(j)}^{-1}, j=$ $1, \ldots, s-1$; from which it follows that $C_{j}=C_{k(j)} \circ E_{j}$ for $j=1, \ldots, s-1$, where $E_{j}$ is a parabolic Möbius transformation with $f\left(E_{j}\right)=f\left(A_{j}\right)$. Condition (7.6.3) means that if the relation $R_{j}^{\prime}$ matches $\left\{\left\{A_{j}^{\prime}\right\}\right\}$ with $\left\{\left\{B_{j}^{\prime}\right\}\right\}$ for $j=$ $s, \ldots, r$, then $\theta\left(A_{j}\right)$ is conjugate to $A_{j}^{\prime}$ or its inverse in $\Gamma_{k(j)}^{\prime}$ and $\theta\left(B_{j}\right)$ is conjugate to $B_{j}^{\prime}$ or its inverse in $\Gamma_{m(j)}^{\prime}$. 
Theorem. For each $t \in \mathbf{D}(\mathscr{G})$, there exists a unique configuration, up to conjugation, of graph type $\mathscr{G}$ that represents the surface $S_{t}$.

Proof. Existence is established by induction on $s$. It should be observed that if $A$ is a parabolic element of a torsion free terminal $b$-group $\Gamma$ with invariant component $\Delta$ that represents a puncture on $\Delta / \Gamma$ that lies to the left of the horocircles determined by $A$, then $E \circ A^{-1} \circ E=A$ is parabolic in $E \Gamma E$ and represents a puncture on $E(\Delta) / E \Gamma E$ that lies to the right of the horocircles determined by $A$, whenever $E$ is elliptic of order 2 and fixes $f(A)$. Uniqueness for nonsingular surfaces is a consequence of a theorem of Maskit [Mt2]: If $\Gamma_{j}$ is a torsion free terminal $b$-group of type $(p, n)$ with invariant component $\Delta_{j}(j=1,2)$ and if $\Delta_{1} / \Gamma_{1}$ is conformally equivalent to $\Delta_{2} / \Gamma_{2}$ as maximally partitioned Riemann surfaces (this means that the image under the equivalence of a partition curve on $\Delta_{1} / \Gamma_{1}$ is freely homotopic to a partition curve on $\left.\Delta_{2} / \Gamma_{2}\right)$, then $\Gamma_{1}$ is conjugate to $\Gamma_{2}$. Uniqueness in the general case is now easily established. The details are left to the reader.

Remark. We describe an alternate construction of the configuration $\Gamma_{t}$. Choose any $t^{*} \in \mathbf{D}_{0}(\mathscr{G})$ with the property that $t_{j}^{*}=t_{j}$ for all $j=1, \ldots, d$, with $t_{j} \neq$ 0 . For $j=1, \ldots, s$, let $S_{(j)}$ be the connected component of $S_{t^{*}}-\left\{a_{k} ; t_{k}=0\right\}$ corresponding to the graph $\mathscr{G}_{j}$. We represent the surface $S_{t^{*}}$ by the group $\Gamma_{i^{*}}$ of graph type $\mathscr{G}$ with invariant component $\Delta$. We choose subgroups $\Gamma_{j}, j=$ $1, \ldots, s$, and parabolic elements $A_{j}, j=1, \ldots, s-1$, as follows. Let $\Delta_{1}^{*}$ be a component of the preimage of $S_{(1)}$ in $\Delta$, let $\Gamma_{1}$ be its stabilizer in $\Gamma_{t^{*}}$ and let $\Delta_{1}$ be the invariant component of $\Gamma_{1}$ (note that $\Delta_{1}^{*} \subset \Delta \subset \Delta_{1}$ ). Then we have $\Gamma_{1}=\Gamma_{t^{1}}$. Choose a parabolic element $A_{1} \in \Gamma_{t^{\prime}}$ that represents the puncture $b_{1}$. Having chosen, for $k=1, \ldots, s-1$, the subgroups $\left\{\Gamma_{1}, \ldots, \Gamma_{k}\right\}$ of $\Gamma_{t^{*}}$ that represent the surfaces $\left\{S_{t^{1}}, \ldots, S_{t^{k}}\right\}$ and the parabolic elements $\left\{A_{1}, \ldots, A_{k}\right\}$ that represent the punctures $\left\{a_{1}, \ldots, a_{k-1}\right\}$ and $\left\{b_{1}, \ldots, b_{k}\right\}$, we proceed to choose the group $\Gamma_{k+1}$ as the (unique) stabilizer of a component of a preimage of $S_{k+1}$ with the puncture $a_{k}$ represented by the parabolic element $A_{k}$ and if $k \leq s-2$, the parabolic element $A_{k+1}$ is chosen as any element of the group $\Gamma_{k+1}$ that represents the puncture $b_{k}$. The relations are constructed from the zero coordinates of the vector $t$. They correspond to parabolic elements that are not conjugate in the component groups of the configuration, but are conjugate in the group $\Gamma_{t^{*}}$.

\section{ONE-DIMENSIONAL MODULI SPACES}

In this section we determine the structure of $N(\Gamma) / \Gamma$ and $\operatorname{Mod} \Gamma$ for $\Gamma$ a torsion free terminal $b$-group of type $(0,4)$ and $(1,1)$. This will lead us to the formula for an isomorphism between $T(0,4)$ and $T(1,1)$ in terms of horocyclic coordinates. In the next section, we will describe the structure of $\operatorname{Mod} \Gamma$ for arbitrary torsion free terminal $b$-groups $\Gamma$. 
8.1. Let $\Gamma=\Gamma_{1}(\alpha)$ be the group of type $(0,4)$ studied in $\S 6.1$. We observe that $A^{1 / 2} \in N(\Gamma)-\Gamma$;

(8.1.1) $A^{1 / 2} \circ A \circ A^{-1 / 2}=A$,

$$
A^{1 / 2} \circ B_{j} \circ A^{-1 / 2}=\left(A \circ B_{j}\right)^{-1}, \quad j=1,2 .
$$

Thus $A^{1 / 2} F_{j} A^{-1 / 2}=F_{j}, j=1,2$. Next we consider

$$
E_{\alpha}=i\left[\begin{array}{ll}
1 & -\alpha \\
0 & -1
\end{array}\right] \text {, }
$$

and observe that

$$
E_{\alpha} \circ A \circ E_{\alpha}^{-1}=A^{-1}, \quad E_{\alpha} \circ B_{1} \circ E_{\alpha}^{-1}=B_{2}^{-1}, \quad E_{\alpha} \circ B_{2} \circ E_{\alpha}^{-1}=B_{1}^{-1} .
$$

Thus $E_{\alpha} \in N(\Gamma)-\Gamma$, and conjugation by $E_{\alpha}$ interchanges $F_{1}$ and $F_{2}$.

Let $E \in N(\Gamma)$. Then $E$ conjugates $A$ to a $\Gamma$-conjugate of $A^{ \pm 1}$. Following $E$ by an element of $\Gamma$, we may assume that $E \circ A \circ E^{-1}=A^{ \pm 1}$. Following $E$ by $E_{\alpha}$, if necessary, we may assume that $E \circ A \circ E^{-1}=A$. It follows that $E$ conjugates $F_{j}$ onto itself (for $j=1,2$ ), and hence $E$ must be a power of $A^{1 / 2}$. We have shown that $N(\Gamma) / \Gamma$ is $\mathbb{Z}_{2} \oplus \mathbb{Z}_{2}$, the Klein four group. We claim that $N(\Gamma)$ is a terminal regular $b$-group of signature $(0,4 ; 2,2,2, \infty){ }^{9}$. To verify this claim, let $F_{3}$ be the triangle group of signature $(0,3 ; 2, \infty, \infty)$ generated by $F=F_{1}$ and $A^{1 / 2}$. Its presentation is

$$
F_{3}=\left\langle A^{1 / 2}, B_{1} ;\left|A^{1 / 2}\right|=\infty=\left|B_{1}\right|,\left|A^{1 / 2} \circ B_{1}\right|=2\right\rangle .
$$

The elementary group $F_{4}$ generated by $A^{1 / 2}$ and $E_{\alpha}$ is euclidean of signature $(0,3 ; 2,2, \infty)$ and has standard presentation

$$
F_{4}=\left\langle A^{1 / 2}, E_{\alpha} ;\left|A^{1 / 2}\right|=\infty,\left|E_{\alpha}\right|=2=\left|A^{1 / 2} \circ E_{\alpha}\right|\right\rangle .
$$

The group $N(\Gamma)$ (see Figure 12 on page 554) is the AFP of $F_{3}$ and $F_{4}$ across $\left\langle A^{1 / 2}\right\rangle$. Its standard presentation is

$$
\begin{array}{r}
N\left(\Gamma_{1}(\alpha)\right)=\left\langle A^{1 / 2}, B_{1}, E_{\alpha} ;\left|B_{1}\right|=\infty,\left|E_{\alpha}\right|=2, \quad\left|A^{1 / 2} \circ B_{1}\right|=2=\left|A^{1 / 2} \circ E_{\alpha}\right|,\right. \\
\left.A^{1 / 2} \text { is accidental parabolic }\right\rangle .
\end{array}
$$

By the results of [BG] and [K2], we may use $\mathrm{T}\left(N\left(\Gamma_{1}\left(\alpha_{0}\right)\right)\right)$ as a model for $\mathbf{T}(0,4)$, where $\alpha_{0}=2 i$, for example. We showed in [K6] that $\operatorname{tr}\left(E_{\alpha} \circ B_{1}\right)$ is a global coordinate for the Teichmüller space $\mathbf{T}\left(N\left(\Gamma_{1}\left(\alpha_{0}\right)\right)\right)$. A simple computation shows that $\operatorname{tr}\left(E_{\alpha} \circ B_{1}\right)=2 i \alpha$.

8.2. A variation of the argument of $\S 8.1$ describes all the geometric automorphisms of $\Gamma=\Gamma_{1}(\alpha)$. Let $\omega \in N_{\mathrm{qc}}(\Gamma)$. Replacing $\omega$ by $\gamma \circ \omega$ or $E_{\alpha} \circ \gamma \circ \omega$ (with $\gamma \in \Gamma$ ), we may assume that $\omega$ conjugates $F_{1}$ onto itself and commutes with $A$. It follows that on $F_{1}, \theta_{\omega}$ is conjugation by $A^{k / 2}$ with $k \in \mathbb{Z}$. Replacing $\omega$ by $A^{-k / 2} \circ \omega$, we may assume that $\omega$ commutes with each element of

\footnotetext{
${ }^{9}$ The signature of a terminal $b$-group is defined in [K6], for example.
} 


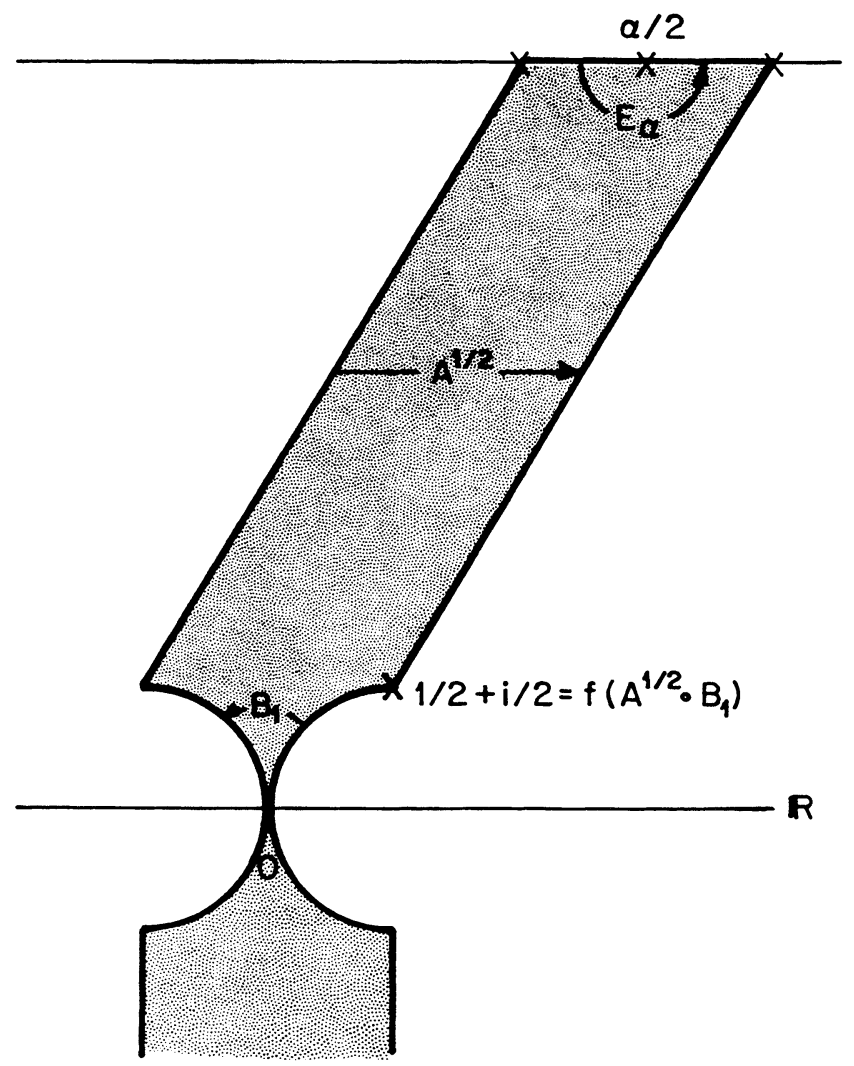

FIGURE 12. A fundamental domain for $N\left(\Gamma_{1}(\alpha)\right)$.

$F_{1}$. Now $\omega$ conjugates $F_{2}$ to $\gamma F_{2} \gamma^{-1}$ for some $\gamma \in F_{2}$. Since $\omega$ commutes with $A \in F_{2}, \gamma$ must be the identity. Thus $\theta_{\omega} \mid F_{2}$ is conjugation by $A^{k / 2}$. It remains to verify that the automorphism $\theta: \Gamma \rightarrow \Gamma$ defined by

$$
\theta(\gamma)= \begin{cases}\gamma & \text { for } \gamma \in F_{1}, \\ A^{1 / 2} \circ \gamma \circ A^{-1 / 2} & \text { for } \gamma \in F_{2},\end{cases}
$$

is geometric. This automorphism $\theta$ is induced by the half Dehn twist about the partition curve $\tilde{a}$ (corresponding to the element $A \in \Gamma$ ). The action of $\theta$ on $\mathbf{T}(0,4)$ in the $\alpha$-coordinates described in $\S 6.1$ is $\alpha \mapsto \alpha+1$. It follows that $e^{2 \pi i \alpha}$ is a complete invariant for the Kleinian group $\Gamma$ and that $\mathbf{R}(\Gamma)$ is complex analytically equivalent to a punctured disc. It is convenient to summarize the above information in the following exact sequence:

$$
\begin{aligned}
1 \rightarrow & N(\Gamma) / \Gamma \rightarrow \operatorname{Mod} \Gamma \rightarrow \operatorname{Aut} \mathbf{T}(\Gamma) ; \\
& \cong \\
\mathbb{Z}_{2} \oplus \mathbb{Z}_{2} &
\end{aligned}
$$

we identify the image of $\operatorname{Mod} \Gamma$ in $\operatorname{Aut} T(\Gamma)$ with the additive group $\mathbb{Z}$, where $1 \in \mathbb{Z}$ corresponds to the half Dehn twist about the partition curve $\tilde{a}$. We note 
that the half Dehn twist permutes exactly two of the four punctures on $\Delta / \Gamma$ (and fixes the other two). The permuted pair of punctures lie on the same side of the partition curve $\tilde{a}$.

8.3. Let $\Gamma=\Gamma_{2}(\tau)$ be the group of type $(1,1)$ described in $\S 6.3$. Let $E \in$ $N(\Gamma)$. Then $E$ maps $\mathbb{H}_{*}^{2}$ onto $\gamma\left(\mathbb{H}_{*}^{2}\right)$ with $\gamma \in \Gamma$. It follows that $\gamma^{-1} \circ E$ maps $\mathbb{H}_{*}^{2}$ onto itself and belongs to $N_{\mathbb{R}}(F)=\operatorname{PSL}(2, \mathbb{Z})$. We replace $E$ by $\gamma^{-1} \circ E$. Now $E \circ A \circ E^{-1}$ is conjugate in $\Gamma$ to $A^{ \pm 1}$, while $E \circ(A \circ B) \circ E^{-1}$ is conjugate in $F$ to $A \circ B$. Following $E$ by an element of $F$, if necessary, we may assume that $E$ commutes with $A \circ B$. Hence $E=(A \circ B)^{k / 2}$ with $k \in \mathbb{Z}$. We compute

$$
\begin{gathered}
C \circ(A \circ B)^{-1 / 2}=i\left[\begin{array}{cc}
1 & -\tau-2 \\
0 & -1
\end{array}\right]=E_{\tau+2} \quad(\text { in } \operatorname{PSL}(2, \mathbb{C})), \\
(A \circ B)^{1 / 2} \circ C \circ(A \circ B)^{-1 / 2}=i\left[\begin{array}{cc}
-2 & 2 \tau+3 \\
-1 & \tau+2
\end{array}\right]=(A \circ B) \circ C^{-1} .
\end{gathered}
$$

We have shown that $N(\Gamma) / \Gamma \cong \mathbb{Z}_{2}$. The element $E_{\tau+2} \in N(\Gamma)$ is elliptic of order 2 and conjugates $A$ to $A^{-1}$. To compute $N(\Gamma)$, we let $F_{5}$ be the group generated by $(A \circ B)^{1 / 2}$ and $F$. It has signature $(0,3 ; \infty, \infty, 2)$. A standard presentation for $F_{5}$ is

$$
F_{5}=\left\langle A^{-1},(A \circ B)^{1 / 2} ;|A|=\infty,\left|(A \circ B)^{1 / 2}\right|=\infty,\left|A^{-1} \circ(A \circ B)^{1 / 2}\right|=2\right\rangle \text {. }
$$

The group $F_{6}$ generated by $E_{\tau+2}=C \circ(A \circ B)^{-1 / 2}$ and $A^{-1}$ is euclidean of signature $(0,3 ; \infty, 2,2)$. Its presentation is

$$
F_{6}=\left\langle A^{-1}, E_{\tau+2} ;\left|A^{-1}\right|=\infty,\left|E_{\tau+2}\right|=2,\left|A^{-1} \circ E_{\tau+2}\right|=2\right\rangle .
$$

The AFP of $F_{5}$ and $F_{6}$ is precisely $N(\Gamma)$. See Figure 13 on page 557. Its standard presentation is

$N\left(\Gamma_{2}(\tau)\right)=\left\langle A^{-1},(A \circ B)^{1 / 2}, E_{\tau+2} ; A^{-1}\right.$ is accidental parabolic, $\left|(A \circ B)^{1 / 2}\right|=\infty$, $\left.\left|A^{-1} \circ(A \circ B)^{1 / 2}\right|=2,\left|E_{\tau+2}\right|=2,\left|A^{-1} \circ E_{\tau+2}\right|=2\right\rangle$.

We show in [K6] that $\operatorname{tr}\left((A \circ B)^{1 / 2} \circ E_{\tau+2}\right)=i \tau$ is a global coordinate for the Teichmüller space of $N\left(\Gamma_{2}\left(\tau_{0}\right)\right)$, where $\tau_{0} \in \mathbf{T}(1,1)$ is arbitrary. For future use, we record the action of two elements of $N(\Gamma)-\Gamma$ on generators for the group $\Gamma$ :

(I) $(A \circ B)^{-1 / 2} \circ A \circ(A \circ B)^{1 / 2}=B, \quad(A \circ B)^{-1 / 2} \circ C \circ(A \circ B)^{1 / 2}=C^{-1} \circ(A \circ B)$.

This automorphism sends $F$ to itself.

(II) $E_{\tau+2} \circ A \circ E_{\tau+2}=A^{-1}, \quad E_{\tau+2} \circ C \circ E_{\tau+2}=(A \circ B) \circ C^{-1}=A \circ C^{-1} \circ A^{-1}$.

This automorphism takes $F$ to $C F C^{-1}$; note that $C\left(\mathbb{H}_{*}^{2}\right) \neq \mathbb{H}_{*}^{2}$.

Remark. The conformal self-map of $\Delta / \Gamma$ induced by either of the above maps corresponds to a half Dehn twist about the puncture on $\Delta / \Gamma$. 
8.4. Let $\omega \in N_{\mathrm{qc}}(\Gamma)$ induce the geometric automorphism $\theta$. Then $\theta(A)$ is conjugate to $A^{ \pm 1}$ in $\Gamma$. Following $\theta$ by conjugation by $E_{\tau+2}$ (if necessary), we may assume that $\theta(A)$ is conjugate to $A$ and (following $\theta$ by a conjugation by an element of $\Gamma)$ that $\theta(A)=A$. Now $\theta(F)$ is a structure subgroup of $\Gamma$ and since $\theta(F) \cap F \supset\langle A\rangle$, either $\theta(F)=F$ or $\theta(F)$ and $F$ stabilize adjacent structure regions. The quasiconformal map $\omega$ fixes the structure loop corresponding to $A$. It cannot reverse the orientation of this loop (otherwise it would conjugate $A$ to $A^{-1}$ ). It follows that $\omega$ fixes each of these two structure regions and hence $\theta(F)=F$. Thus $\theta \mid F \in N_{\mathbb{R}}(F)$ with $\theta(A)=A$. Hence $\theta \mid F$ is conjugation by $A^{k / 2}$ for some $k \in \mathbb{Z}$. If $k$ is odd, then $\theta$ takes $B$ to an element conjugate to $B^{-1} \circ A^{-1}$. But $\theta(B)$ must be conjugate to $B^{ \pm 1}$ in $\Gamma$. It follows that $k$ is even. Following $\theta$ by conjugation by $A^{k / 2} \in \Gamma$, we may assume that $\theta(A)=A, \theta(B)=B$; that is, $\theta$ acts trivially on $F$. Now $\theta$ is determined completely by its value on $C$. Let $\widetilde{C}=\theta(C)$. Then from (6.3.1) we conclude $\widetilde{C} \circ\left(B^{-1}\right) \circ \widetilde{C}^{-1}=A$. It follows that $\left(C \circ \widetilde{C}^{-1}\right) \circ A \circ\left(\widetilde{C} \circ C^{-1}\right)=A$. Since both $C$ and $\widetilde{C}$ belong to $\Gamma$ we conclude that $\widetilde{C}=A^{k} \circ C$, for some $k \in \mathbb{Z}$. Existence of these geometric automorphisms is guaranteed by the Dehn twists around the curve $\tilde{a}$. The action of $\theta$ on $\mathbf{T}(1,1)$ in the $\tau$ coordinate is $\tau \mapsto \tau+2$. Hence $e^{\pi i \tau}$ is a complete invariant for the conjugacy class of $\Gamma$ in $\operatorname{PSL}(2, \mathbb{C})$.

We describe (up to inner automorphisms) an arbitrary geometric automorphism $\theta$ of $\Gamma$. These automorphisms are of two types:

(I) $\theta$ is the identity on $F$ and $\theta(C)=A^{k} \circ C$ for some $k \in \mathbb{Z}$, or

(II) $\theta$ conjugates $F$ to $C F C^{-1}, \theta(A)=A^{-1}$ and $\theta(C)=A^{k} \circ C^{-1} \circ A^{-1}$, for some $k \in \mathbb{Z}$.

Again, an exact sequence encodes the above data:

$$
1 \rightarrow \underset{\substack{N(\Gamma) / \Gamma \\ \cong \\ \mathbb{Z}_{2}}}{\cong} \operatorname{Mod} \Gamma \rightarrow \operatorname{Aut} \mathbf{T}(\Gamma) .
$$

We identify the image of $\operatorname{Mod} \Gamma$ in $\operatorname{Aut} T(\Gamma)$ with $2 \mathbb{Z}$, with $2 \in \mathbb{Z}$ corresponding to the Dehn twist about the partition curve $\tilde{a}$ on $\Delta / \Gamma$.

8.5. Let $\Gamma$ be a terminal $b$-group of signature $(0,4 ; 2,2,2, \infty)$. Without loss of generality we may take $\Gamma=N\left(\Gamma_{2}(\tau)\right)$ of $\S 8.3$. Let $\Delta$ be the invariant component of $\Gamma$. Let $\omega \in N_{\mathrm{qc}}(\Gamma)$. Then $\omega$ induces a self-map of $\Delta / \Gamma$ that preserves the partition curve as well as each part (because the two parts have different signature). Thus $\theta_{\omega}(A)$ is conjugate to $A^{ \pm 1}$. Now $\theta_{\omega}(A)$ must be conjugate to $A$ (otherwise the two parts of $\Delta / \Gamma$ would be interchanged). Replacing $\omega$ by $\gamma \circ \omega$ for some $\gamma \in \Gamma$, we achieve $\theta_{\omega}(A)=A$. It follows that $\theta_{\omega}\left(F_{j}\right)=F_{j}, j=5$ and 6 , and thus there exists $E_{j} \in N\left(F_{j}\right)$ such that $\theta_{\omega}\left|F_{j}=\theta_{E_{j}}\right| F_{j}$. The map that $E_{5}$ induces on $\mathbb{H}_{*}^{2} / F_{5}$ fixes the image of the elliptic fixed point and the puncture corresponding to $A$; and thus is the identity. 


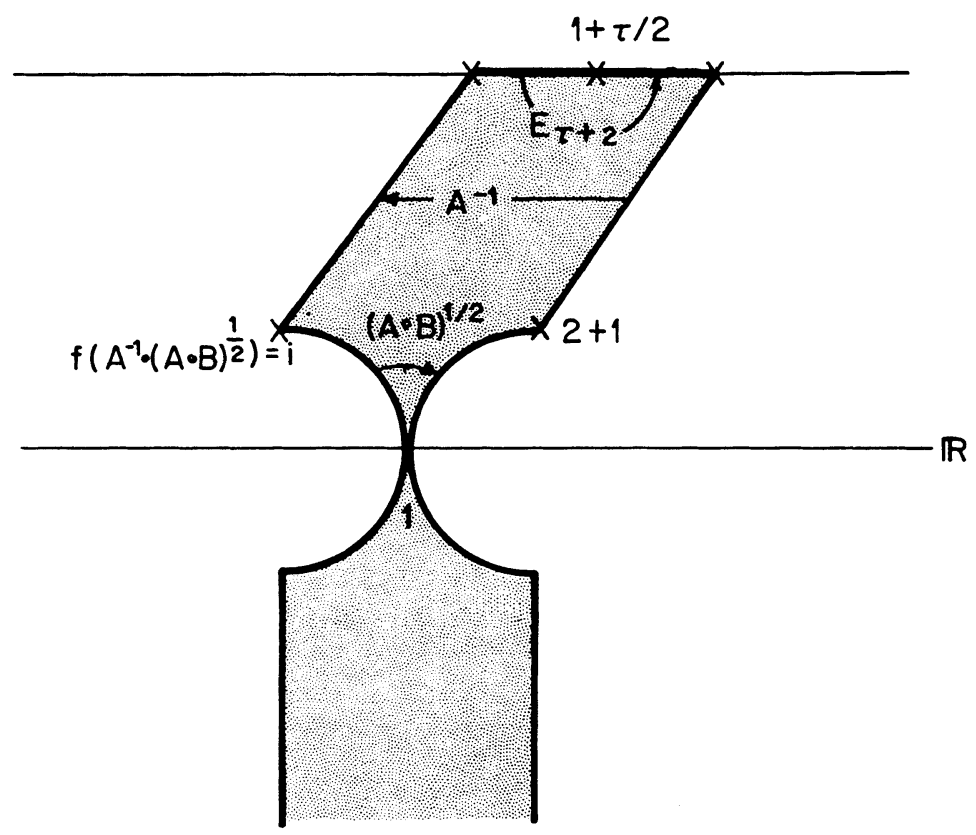

FIGURE 13. A fundamental domain for $N\left(\Gamma_{2}(\tau)\right)$.

It follows that we may assume that $E_{5}=I$. Since $E_{6}$ conjugates $A$ to itself, $E_{6}=\left[\begin{array}{ll}1 & b \\ 0 & 1\end{array}\right]$ for some $b \in \mathbb{C}^{*}$. Since $E_{6} \circ E_{\tau+2} \circ E_{6}^{-1}$ must be of the form

$$
z \mapsto(-1)^{k}\left(z-\frac{\tau}{2}-1\right)+\left(\frac{\tau}{2}+1\right)+2 n, \quad k, n \in \mathbb{Z},
$$

we conclude that $b=n$ or (equivalently) that $E_{6}=A^{n / 2}$. We have shown that every geometric automorphism of $\Gamma$ is conjugate to an automorphism $\theta$ that satisfies

$$
\theta(A)=A, \quad \theta\left((A \circ B)^{1 / 2}\right)=(A \circ B)^{1 / 2}, \quad \theta\left(E_{\tau+2}\right)=A^{n} \circ E_{\tau+2}, \quad n \in \mathbb{Z} .
$$

As before, these are precisely the Dehn twists about the partition curve. The above considerations have also shown that $N(\Gamma)=\Gamma$.

As before, $\operatorname{tr}\left((A \circ B)^{1 / 2} \circ E_{\tau+2}\right)=i \tau$ is a global coordinate for $\mathbf{T}\left(\Gamma_{0}\right)$, where $\Gamma_{0}$ is an arbitrary $b$-group of signature $(0,4 ; 2,2,2, \infty)$. The action of $\theta$ on $\mathbf{T}\left(\Gamma_{0}\right)$ in these coordinates is given by $\tau \mapsto \tau+2 n$. Hence $e^{\pi i \tau}$ is a complete invariant for the conjugacy class of $\Gamma$. (Note that $(A \circ B)^{1 / 2} \circ C \circ(A \circ B)^{-1 / 2}$ is conjugate to $C$ in $\Gamma$.)

8.6. We have seen that for all $\tau \in \mathbf{T}(1,1), \Gamma_{2}(\tau) \subset \Gamma_{1}(\tau)$ and $\tau \in \mathbf{T}(0,4)$. The groups $N\left(\Gamma_{1}(\tau)\right)$ and $N\left(\Gamma_{2}(\tau)\right)$ are never conjugate since their invariants $\left(e^{2 \pi i \tau}\right.$ and $\left.e^{\pi i \tau}\right)$ are different. Given $\alpha \in \mathbf{T}(0,4)$, then (recall $\S 6.3$, Remarks (2) and (3)) we can construct a group $\Gamma_{2}(\alpha)$ that is a candidate for a point in $\mathbf{T}(1,1)$. 
Theorem. The map $\alpha \mapsto \tau=2 \alpha$ establishes a complex analytic isomorphism between $\mathbf{T}(0,4)$ and $\mathbf{T}(1,1)$.

Proof. The map from $\mathbf{T}(0,4)$ to $\mathbf{T}(1,1)$ is constructed as follows. Let $\alpha \in$ $\mathbf{T}(0,4)$. Find $\tau \in \mathbf{T}(1,1)$ so that $N\left(\Gamma_{1}(\alpha)\right)=N\left(\Gamma_{2}(\tau)\right)$. For any $\alpha_{0} \in$ $\mathbf{T}(0,4)$ and any $\tau_{0} \in \mathbf{T}(1,1)$, the inclusions $\Gamma_{1}\left(\alpha_{0}\right) \subset N\left(\Gamma_{1}\left(\alpha_{0}\right)\right)$ and $\Gamma_{2}\left(\tau_{0}\right) \subset$ $N\left(\Gamma_{2}\left(\tau_{0}\right)\right)$ induce isomorphisms

$$
\mathbf{T}\left(N\left(\Gamma_{1}\left(\alpha_{0}\right)\right)\right) \rightarrow \mathbf{T}\left(\Gamma_{1}\left(\alpha_{0}\right)\right), \quad \mathbf{T}\left(N\left(\Gamma_{2}\left(\tau_{0}\right)\right)\right) \rightarrow \mathbf{T}\left(\Gamma_{2}\left(\tau_{0}\right)\right) .
$$

Hence our recipe is a well-defined holomorphic bijection of $\mathbf{T}(0,4)$ onto $\mathbf{T}(1,1)$. We have seen that the first of the maps (8.6.1) in the $\alpha$-coordinates is multiplication by $\frac{1}{2}$, while the second is the identity in the $\tau$-coordinates. Indeed, it is easy to see that $N\left(\Gamma_{1}(\alpha)\right)$ and $N\left(\Gamma_{2}(2 \alpha)\right)$ are conjugate groups. Let $E(z)=2 z+1$; then

$E \circ A^{1 / 2} \circ E^{-1}=A, \quad E \circ B_{1} \circ E^{-1}=(A \circ B)^{-1 / 2}, \quad E \circ E_{\alpha} \circ E^{-1}=E_{2 \alpha+2}$.

Corollary 1. For $\alpha \in \mathbf{T}(0,4), \Gamma_{2}(\alpha)$ is a terminal b-group of signature (1, 1; $\infty)$ if and only if $2 \alpha \in \mathbf{T}(1,1)$.

Corollary 2. If $\tau \in \mathbf{T}(1,1)$, then $\operatorname{Im} \tau>1$.

Proof. We saw in $\S 6.1$ that for $\alpha \in \mathbf{T}(0,4)$ we must have $\operatorname{Im} \alpha>\frac{1}{2}$.

8.7. Our work on one-dimensional moduli spaces has immediate applications to more complicated groups.

Theorem. Let $\Gamma$ be a torsion free terminal b-group of type $(0,4),(1,1),(1,2)$ or $(2,0)$. Then $N(\Gamma) / \Gamma$ always contains an element of order 2 corresponding to the hyperelliptic involution on $\Delta / \Gamma$; this element (viewed as an element of Mod $\Gamma$ ) acts trivially on $T(\Gamma)$.

Proof. The $(0,4)$ and $(1,1)$ cases have been treated already. Assume that $\Gamma$ is of type $(2,0)$. Then the graph corresponding to $\Gamma$ is given by either Figure 10 or 11 and the structure of $\Gamma$ is described by the illustrative examples in $\S 7.5$.

The Figure 10 example. The Möbius transformation $A_{2}^{1 / 2}$ conjugates $G_{2}$ onto itself (see equation (8.1.1)); as a matter of fact

$$
A_{2}^{1 / 2} \circ A_{1} \circ A_{2}^{-1 / 2}=B, \quad A_{2}^{1 / 2} \circ B_{2}^{-1} \circ A_{2}^{-1 / 2}=B_{2} \circ A_{2}^{-1} .
$$

Further, $A_{2}^{1 / 2}$ conjugates $G_{1}$ and $G_{3}$ onto themselves (see the end of $\S 8.3$, case (I));

$$
A_{2}^{1 / 2} \circ C_{1} \circ A_{2}^{-1 / 2}=C_{1}^{-1} \circ A_{2}^{-1}, \quad A_{2}^{1 / 2} \circ C_{3} \circ A_{2}^{-1 / 2}=C_{3}^{-1} \circ A_{2}^{-1} .
$$

The Figure 11 example. Let $E=E_{\tau}$ as defined by (8.1.2). Then $E$ conjugates $G_{1}$ onto itself. Direct calculations show that

$$
E \circ C_{j} \circ E^{-1}=C_{j}^{-1}, \quad j=2,3 .
$$


Remark. The Möbius transformation $E$ satisfies $E^{2}=I, E \circ B \circ E^{-1}=B_{1}$, $E \circ B_{1} \circ E^{-1}=B$. Hence

$$
E \circ C_{2} \circ E \circ B_{1} \circ E \circ C_{2}^{-1} \circ E=E \circ C_{2} \circ B \circ C_{2}^{-1} \circ E=E \circ B_{1}^{-1} \circ E=B^{-1} \text {. }
$$

Thus $E \circ C_{2} \circ E$ and $C_{2}^{-1}$ conjugate $B_{1}$ to $B^{-1}$. Since $\operatorname{tr}\left(E \circ C_{2} \circ E\right)=\operatorname{tr} C_{2}^{-1}$, we would like to conclude that $E \circ C_{2} \circ E=C_{2}^{-1}$; orientation consideration plus Lemma 12.3 allow us to do so. It is simpler just to compute (using a symbolic manipulation program such as MACSYMA).

To finish the proof of the theorem, we must consider groups of type $(1,2)$. The admissible graphs of type $(1,2)$ are subgraphs of graphs of type $(2,0)$, and have already been considered in the above proof.

\section{MODULI SPACES FOR TORSION FREE TERMINAL $b$-GROUPS}

This section is devoted to the study of the moduli or Riemann spaces of torsion free terminal $b$-groups and admissible graphs.

9.1. Let $\Gamma$ be a torsion free terminal $b$-group of graph type $\mathscr{G}$ with invariant component $\Delta$ and ordinary set $\Omega$. We use the same notation and conventions as in §7.2. To study the moduli space $\mathbf{R}(\Gamma)=\mathbf{T}(\Gamma) / \operatorname{Mod} \Gamma$, we choose representatives $A_{1}, \ldots, A_{d}$ of the nonconjugate, primitive, accidental parabolic elements in $\Gamma$, and modular subgroups $G_{j} \subset \Gamma$ such that $A_{j}$ is accidental parabolic in $G_{j}, j=1, \ldots, d$. The parabolic motion $A_{j}$ and the group $G_{j}$ correspond to the edge $a_{j}$ on $\mathscr{G}$. Let $S^{k}, k=1,2, \ldots, v$, be the parts of $S_{0}=\Delta / \Gamma-\Sigma$, where $\Sigma=\left\{a_{1}, \ldots, a_{d}\right\}$ is the partition on $S=\Delta / \Gamma$ determined by the accidental parabolic elements in $\Gamma$. We assume, as usual, that $d>0$.

Let $\omega \in N_{\mathrm{qc}}(\Gamma)$. The quasiconformal map $\omega$ fixes $\Delta$, conjugates $\Gamma$ onto itself and takes accidental parabolics onto accidental parabolics. Hence the induced map $\tilde{\omega}: \Delta / \Gamma \rightarrow \Delta / \Gamma$ has the property that, for $j=1, \ldots, d, \tilde{\omega}\left(a_{j}\right)$ is freely homotopic to $a_{\sigma_{2}(j)}$, where $\sigma_{2}$ is a permutation of $\{1, \ldots, d\}$. We may assume (see, for example, [B10]), without loss of generality, that $\tilde{\omega}\left(a_{j}\right)=a_{\sigma_{2}(j)}^{ \pm 1}$, $j=1, \ldots, d$. It follows that $\tilde{\omega}$ takes parts of $S_{0}$ onto other parts; that is, there exists a permutation $\sigma_{1}$ of $\{1, \ldots, v\}$ such that $\tilde{\omega}\left(S^{k}\right)=S^{\sigma_{1}(k)}$, $k=1, \ldots, v$. Since $\tilde{\omega}$ also permutes the punctures of $\Delta / \Gamma$, it maps the set of phantom edges of $\mathscr{G}$ onto itself. Thus we have produced an automorphism $\sigma=$ $g(\omega)$ of the augmented graph $\mathscr{G}$ : an element of Aut $\mathscr{G}$. The automorphism $g(\omega)$ depends only on the equivalence class of $\omega$ in $\operatorname{Mod} \Gamma$. Hence we have a well-defined group homomorphism

$$
g: \operatorname{Mod} \Gamma \rightarrow \operatorname{Aut} \mathscr{G} .
$$

Remark. Both $\sigma_{2}$ and $\sigma_{1}$ can be the identity without $\sigma$ being the identity because $\sigma$ also acts on the phantom edges and can reverse the orientation of edges. If $a$ is an edge of $\mathscr{G}$ that joins a vertex $S$ to itself, then there exists an automorphism $\sigma$ of $\mathscr{G}$ that preserves all the other edges and sends $a$ to its 
inverse. If $a^{\prime}$ is the other edge or phantom edge emanating from $S$, then the half Dehn twist about $a^{\prime}$ projects (via $g$ ) onto $\sigma$.

Caution. In defining the homomorphism from $\operatorname{Mod} \Gamma$ to $\operatorname{Aug} \mathscr{G}$ we have used the fact that the elements of the modular group arise from orientation preserving maps. An edge in the graph $\mathscr{G}$ determines a cylinder $\mathscr{A}$ on the surface $S$ as well as a central curve $a$ on $\mathscr{A}$. Let $\omega$ be an orientation preserving automorphism of $\mathscr{A}$ that fixes the curve $a$. Then $\omega$ reverses the orientation of $a$ if and only if it interchanges the components of $\mathscr{A}-\{a\}$. Orientation reversing automorphisms do not have this property. A nontrivial (in the sense that it is not a product of Dehn twists about partition curves) orientation reversing self-map of $S$ may induce the trivial automorphism of $\mathscr{G}$.

9.2. As before, we let $\mathscr{G}_{k}$ be the restriction of $\mathscr{G}$ to the edges $a_{1}, \ldots, a_{k}$ for $k=1, \ldots, d$. Then $\mathscr{G}_{k}$ is connected and admissible since $\mathscr{G}$ has a semicanonical ordering for its edges. We let $\Gamma_{k}$ be the subgroup of $\Gamma$ corresponding to the graph $\mathscr{G}_{k}$. At this point it is important to recall the conventions of $\S 3.2$. Choose structure loops $\tilde{a}_{1}, \ldots, \tilde{a}_{d}$ that cover the partition curves $a_{1}, \ldots, a_{d}$ (see $\S 5.1$ ). We choose these loops so that for $j=1, \ldots, d$, the modular region $D_{j}$ that covers the modular part $T_{j}$ contains $\tilde{a}_{j}$ and so that $D_{j} \cap D_{i}$ is nonempty for some $i$ with $1 \leq i \leq j-1$. We may assume that $G_{j}$ is the modular subgroup of $\Gamma$ that stabilizes $D_{j}$.

9.3. We define a homomorphism (recall (9.1.1))

$$
q: \operatorname{ker} g \rightarrow \operatorname{Mod} G_{1} \times \cdots \times \operatorname{Mod} G_{d}
$$

as follows. If $\theta=\theta_{\omega}, \omega \in N_{\mathrm{qc}}(\Gamma), \theta \in \operatorname{ker} g$, then $\theta\left(A_{j}\right)$ is conjugate in $\Gamma$ to $A_{j}$. It follows that $\theta\left(G_{j}\right)=E_{j} G_{j} E_{j}^{-1}$ for some $E_{j} \in \Gamma$. Then $\theta_{E_{j}^{-1}} \circ \theta$ conjugates $G_{j}$ onto itself and induces an element of $\operatorname{Mod} G_{j}$. If $E_{j} G_{j} E_{j}^{-1}=\widetilde{E}_{j} G_{j} \widetilde{E}_{j}^{-1}$ for some other element $\widetilde{E}_{j} \in \Gamma$, then $\widetilde{E}_{j}^{-1} \circ E_{j} \in G_{j}$ and $\theta_{\widetilde{E}_{j}^{-1}} \circ \theta=\theta_{\widetilde{E}_{j}^{-1} \circ E_{j}} \circ \theta_{E_{j}^{-1}} \circ \theta$ is equivalent in $\operatorname{Mod} G_{j}$ to $\theta_{E_{j}^{-1}} \circ \theta$. One shows similarly that replacing $\omega$ by $E \circ \omega$ for some $E \in \Gamma$ results in the same element of $\operatorname{Mod} G_{j}$. Thus we have a well-defined mapping $q$ (between the groups in (9.3.1)).

The mapping $q$ is a homomorphism. For $\omega_{1}$ and $\omega_{2} \in N_{\mathrm{qc}}(\Gamma)$, let $\theta_{i}=\theta_{\omega_{i}}$ and assume that $\theta_{i}\left(G_{j}\right)=E_{j}^{(i)} G_{j}\left(E_{j}^{(i)}\right)^{-1}$. Then

$$
\left(\theta_{2} \circ \theta_{1}\right)\left(G_{j}\right)=\theta_{2}\left(E_{j}^{(1)} G_{j}\left(E_{j}^{(1)}\right)^{-1}\right)=\theta_{2}\left(E_{j}^{(1)}\right) \circ E_{j}^{(2)} G_{j}\left(E_{j}^{(2)}\right)^{-1} \circ \theta_{2}\left(E_{j}^{(1)}\right)^{-1},
$$

and for $\gamma \in \Gamma$,

$$
\begin{aligned}
& \theta_{\left(E_{j}^{(2)}\right)^{-1}} \circ \theta_{2} \circ \theta_{\left(E_{j}^{(1)}\right)^{-1}} \circ \theta_{1}(\gamma)=\left(E_{j}^{(2)}\right)^{-1} \circ \theta_{2}\left(\left(E_{j}^{(1)}\right)^{-1} \circ \theta_{1}(\gamma) \circ E_{j}^{(1)}\right) \circ E_{j}^{(2)} \\
& =\left(E_{j}^{(2)}\right)^{-1} \circ \theta_{2}\left(E_{j}^{(1)}\right)^{-1} \circ \theta_{2}\left(\theta_{1}(\gamma)\right) \circ \theta_{2}\left(E_{j}^{(1)}\right) \circ E_{j}^{(2)} \\
& =\left(\theta_{\left[\theta_{2}\left(E_{j}^{(1)}\right) \circ E_{j}^{(2)}\right]^{-1}} \circ \theta_{2} \circ \theta_{1}\right)(\gamma) \text {; }
\end{aligned}
$$


from which it follows that $q\left(\theta_{2} \circ \theta_{1}\right)=q\left(\theta_{2}\right) q\left(\theta_{1}\right)$.

Let $\theta \in \operatorname{ker} g$. Without loss of generality $\theta\left(A_{1}\right)=A_{1}$. Assume $q(\theta)=I$. Then $E_{1}=I$ and $\theta \mid G_{1}=\theta_{B_{1}}$ with $B_{1} \in G_{1}$. It follows that $B_{1}$ is a power of $A_{1}$ and that without loss of generality we may assume that $B_{1}=I$. Assume by induction that $\theta \mid G_{j}$ is the identity for $j=1, \ldots, k, k<d$. Then $A_{k+1}$ belongs to $\Gamma_{k}$ and it follows that $\theta\left(A_{k+1}\right)=A_{k+1}$. Since this implies that $\theta\left(G_{k+1}\right)=G_{k+1}$, it follows that $\theta \mid G_{k+1}$ is conjugation by a power of $A_{k+1}$. Since $G_{k+1}$ intersects nontrivially some $G_{j}$ with $1 \leq j \leq k$, we conclude that $\theta \mid G_{k+1}$ is the identity. Thus $q$ is a monomorphism.

9.4. To describe the image of $q$ it is convenient to define $\operatorname{Mod}_{0} \Gamma=\operatorname{ker} g$. Note that each $G_{i}(1 \leq i \leq d)$ also has a graph (of type $(0,4)$ or $(1,1)$ ) associated to it. Hence the subgroups $\operatorname{Mod}_{0} G_{i}$ are well defined. It is obvious that

$$
q: \operatorname{Mod}_{0} \Gamma \rightarrow \operatorname{Mod}_{0} G_{i} \times \cdots \times \operatorname{Mod}_{0} G_{d} .
$$

Now, $\operatorname{Mod}_{0} G_{i}$ acts effectively on $\mathbf{T}\left(G_{i}\right)$ (see ( $\S 8.2$ and 8.4) and consists of the Dehn twists about the partition curve $a_{i}$; thus we can identify $\operatorname{Mod}_{0} G_{i} \cong 2 \mathbb{Z}$. It follows that the map $q$ of (9.4.1) is surjective and that $\operatorname{Mod}_{0} \Gamma$ is the free abelian group of rank $d$, consisting of the products of the Dehn twists about the partition curves.

Proposition. The quotient space $\mathbf{T}(\Gamma) / \mathrm{ker} g$ is biholomorphically equivalent to a domain in a product of $d$ punctured discs. If $\tau=\left(\tau_{1}, \ldots, \tau_{d}\right)$ are horocyclic coordinates on $\mathbf{T}(\Gamma)$, then the plumbing coordinates (defined in §7.3) $t=\left(t_{1}, \ldots, t_{d}\right)$ are coordinates on the quotient space.

Theorem. We have $\mathbf{D}_{0}(\mathscr{G}) \cong \mathbf{T}(\Gamma) / \operatorname{ker} g$; hence $\mathbf{D}_{0}(\mathscr{G})$ is a domain of holomorphy.

Proof. The isomorphism follows from the description of the construction algorithm for $\Gamma$ (see $\S 7.5)$. The fact that $\mathbf{D}_{0}(\mathscr{G})$ is a domain of holomorphy then follows from an observation of Hejhal [H1].

9.5. We need the following technical result.

Proposition. Let $t_{0} \in \mathbf{D}_{0}(\mathscr{G})$ and let $a_{k}(d<k \leq d+n)$ be a phantom edge on $\mathscr{G}$. For $t \in \mathbf{D}(\mathscr{G})$, let $z(t)$ be a horocyclic coordinate on $S_{t}$ at the puncture corresponding to $a_{k}$. Assume that the image of $z\left(t_{0}\right)$ contains the horodisc of radius $r_{0}>0$. Let $0<\varepsilon<r_{0}$. There exists a $\delta>0$, that depends only on $t_{0}$ and $\mathscr{G}$, such that for $\left|t-t_{0}\right|<\delta$, the image of $z(t)$ contains the horodisc of radius $r_{0}-\varepsilon$.

Remarks. (1) The reader should review the discussion of $\$ 3.6$.

(2) There are exactly two choices for the coordinate $z(t)$; they differ by a minus sign.

Proof of proposition. Let $\Gamma$ be a terminal $b$-group of graph type $\mathscr{G}$ that represents, on its invariant component $\Delta$, the surface $S_{t_{0}}: \Delta / \Gamma \cong S_{t_{0}}$. Without 
loss of generality, the group $F$ (of $\S 1.2$ ) is a structure subgroup of $\Gamma$ that contains the parabolic element $A$ (given by (1.2.2)) that represents the puncture corresponding to the phantom edge $a_{k}$. We may assume that $\Delta$ contains the half-plane $\mathbb{H}^{2}+i \alpha_{0}$ (with $\alpha_{0} \in \mathbb{R}^{+}, \alpha_{0}>\frac{1}{2}$ ) that is precisely invariant under $\langle A\rangle$ in $\Gamma$ (we may choose $r_{0}=e^{-\alpha_{0} \pi}$ ). A neighborhood of the identity isomorphism in $\mathbf{T}(\Gamma)$ consists of the equivalence classes $[w]$ of normalized (at $0,1, \infty) \Gamma$-compatible $K$-quasiconformal automorphisms $w$ of $\hat{\mathbb{C}}$ (see $\S 4.1$ ). It follows that $w \Gamma w^{-1} \supset F$ for all $[w] \in \mathbf{T}(\Gamma)$ and that $z=e^{\pi i \zeta}$ is the horocyclic coordinate on each of our surfaces $S_{t}, t \in \mathbf{D}_{0}(\mathscr{G}), S_{t}=w(\Delta) / w \Gamma w^{-1}$.

If $w$ is a $K$-quasiconformal automorphism of $\hat{\mathbb{C}}$, then for each $\zeta \in \mathbb{C}-$ $\{0,1\}, d(w(\zeta), \zeta) \leq \log K$; here $d(\cdot, \cdot)$ is the Poincaré metric on $\mathbb{C}-\{0,1\}$. See, for example, Ahlfors [A2] or $\mathrm{Kra}[\mathrm{K} 3]$. The domain $w\left(\mathbb{H}^{2}+i \alpha_{0}\right)$ is precisely invariant under $\langle A\rangle$ in $w \Gamma w^{-1}$. Hence it suffices to show that for $K$ sufficiently close to $1, w\left(\mathbb{H}^{2}+i \alpha_{0}\right) \supset \mathbb{H}^{2}+i \alpha, \alpha \in \mathbb{R}^{+}$, with $\alpha$ dependent only on $K$. This follows from the fact that $w \circ A=A \circ w$ for all $[w] \in \mathbf{T}(\Gamma)$. Thus

$$
\sup \left\{\operatorname{Im} w\left(x+i \alpha_{0}\right) ; x \in \mathbb{R}\right\}=\sup \left\{\operatorname{Im} w\left(x+i \alpha_{0}\right) ; 0 \leq x \leq 2\right\} .
$$

We leave it to the reader to estimate $\delta$ in terms of $\alpha_{0}$.

9.6. Proposition. The following is a short exact sequence of groups and group homomorphisms:

$$
0 \rightarrow \operatorname{ker} g \rightarrow \operatorname{Mod} \Gamma \stackrel{g}{\rightarrow} \operatorname{Aug} \mathscr{G} \rightarrow 0 ;
$$

it does not split.

Proof. Surjectivity of $g$ and the nonsplitting are the only issues. View $\mathscr{G}$ as embedded in $\mathbb{R}^{3}$; then a model for $S$ is the boundary of a regular neighborhood of $\mathscr{G}$ in $\mathbb{R}^{3}$. An automorphism $\sigma$ of $\mathscr{G}$ defines a homeomorphism of $\mathscr{G}$ onto itself. It can be extended radially to an orientation preserving automorphism of $S$. This automorphism preserves the partition curves. It follows that $g$ is surjective. To show that the sequence does not split, consider the automorphism $\sigma$ of the graph $\mathscr{G}$ of type $(0,4)$ (see Figure 6 ) defined by

$$
\sigma\left(a_{j}\right)=a_{j}, \quad j=1,2,3, \quad \sigma\left(a_{4}\right)=a_{5}, \quad \sigma\left(a_{5}\right)=a_{4} .
$$

This element of order 2 of Aut $\mathscr{G}$ is the image under $g$ of the half Dehn twist $\tilde{\omega}$ about the partition curve $a_{1}$ (see $\S 8.2$ ). The element $\tilde{\omega}$ is of infinite order in $\operatorname{Mod} \Gamma$. Further, no element of finite order in $\operatorname{Mod} \Gamma$ can map onto $\sigma$. The general case is handled similarly.

Remarks. (1) In $\S 9.10$ we will obtain a second proof of the surjectivity of $g$.

(2) In general, a half Dehn twist about the partition curve on a spherical end of $\Delta / \Gamma$ is an element of infinite order in $\operatorname{Mod} \Gamma$; it is sent by $g$ onto an element of order 2 in Aut $\mathscr{G}$. Similarly, if $T$ is an elliptic end on $\Delta / \Gamma \neq T$, and $a$ is the partition curve on $\Delta / \Gamma$ that bounds $T$, then the half Dehn twist $\tilde{\omega}$ about $a$ is an element of infinite order in $\operatorname{Mod} \Gamma$ that is sent to an involution 
in Aut $\mathscr{G}$. If $\Delta / \Gamma=T$, then $\tilde{\omega}$ induces an element of order 2 of $\operatorname{Mod} \Gamma$ (that acts trivially on $\mathbf{T}(\Gamma)$ ); see also $\S \S 8.3$ and 9.9 .

9.7. To study the action of Aut $\mathscr{G}$ on $\mathbf{D}(\mathscr{G})$ and $\mathbf{D}_{0}(\mathscr{G})$, we start with the following

Definition. The Riemann space of the graph $\mathscr{G}$ is $\mathbf{R}(\mathscr{G})=\mathbf{D}(\mathscr{G}) /$ Aut $\mathscr{G}$.

Theorem. Both $\mathbf{R}(\Gamma)$ and $\mathbf{R}(\mathscr{G})$ are d-dimensional complex analytic orbifolds. Furthermore, $\mathbf{R}(\Gamma) \cong \mathbf{D}_{0}(\mathscr{G}) /$ Aut $\mathscr{G}$.

Proof. Aut $\mathscr{G}$ is a finite group acting on $\mathbf{D}_{0}(\mathscr{G})$ as complex analytic automorphisms. Since $\mathbf{D}_{0}(\mathscr{G})$ is a complex analytic manifold, we conclude that $\mathbf{D}_{0}(\mathscr{G}) /$ Aut $\mathscr{G}$ is a complex orbifold. From Theorem 9.4 and the exact sequence (9.6.1), we conclude that $\mathbf{D}_{0}(\mathscr{G}) /$ Aut $\mathscr{G} \cong \mathbf{T}(\Gamma) / \operatorname{Mod} \Gamma \cong \mathbf{R}(\Gamma)$.

\subsection{The study of $\mathbf{R}(\mathscr{G})$ is based on the following}

Theorem. For every admissible graph $\mathscr{G}, \mathbf{D}(\mathscr{G})$ is a contractible bounded domain of holomorphy in $\mathbb{C}^{d}$.

Proof. We first observe that $\mathbf{D}(\mathscr{G})$ is a domain in $\mathbb{C}^{d}$. We know that $\mathbf{D}_{0}(\mathscr{G}) \subset$ $\mathbf{D}(\mathscr{G})$ is a domain. Let $t=\left(t_{1}, \ldots, t_{d}\right) \in \mathbf{D}(\mathscr{G})-\mathbf{D}_{0}(\mathscr{G})$. Then one or more of the coordinates $t_{j}$ must be zero and these correspond to nodes on $S_{t}$. We can certainly vary each of the nonzero coordinates. The plumbing constructions corresponding to a compact subset of $\mathbf{D}_{0}(\mathscr{G})$ can be restricted to annuli that do not intersect horodiscs about the punctures corresponding to nodes as a consequence of Proposition 9.5. Thus starting with a surface $S_{t}$ with nodes, we can vary the nonzero components of $t$ (viewed as elements of $\mathbf{D}_{0}\left(\mathscr{G}^{\prime}\right)$ for some allowable subgraph $\mathscr{G}^{\prime}$ of $\mathscr{G}$ obtained by breaking the edges $a_{j}$ in $\mathscr{G}$ for which $t_{j}=0$ ) in a compact set. We can then vary the zero components independently of the previous variations using fixed horodiscs about the pairs of punctures corresponding to the nodes. Thus a neighborhood of $t$ in $\mathbb{C}^{d}$ is contained in $\mathbf{D}(\mathscr{G})$.

We observe that every closed curve in $\mathbf{D}_{0}(\mathscr{G})$ is homotopic in $\mathbf{D}_{0}(\mathscr{G})$ to a closed curve in $\left\{t \in \mathbf{D}_{0}(\mathscr{G}) ;|t|<e^{-2 \pi}\right\}$. This follows from the following facts:

(1) $\mathbf{D}_{0}(\mathscr{G}) \cong \mathbf{T}(p, n) /(2 \mathbb{Z})^{d}$,

(2) $\left\{t \in \mathbf{D}_{0}(\mathscr{G}) ;|t|<e^{-2 \pi}\right\}$

$$
\cong\left\{\tau \in \mathbb{C}^{d} ; \operatorname{Im} \tau_{j}>2 \text { for } j=1,2, \ldots, d\right\} /(2 \mathbb{Z})^{d},
$$

(3) $\mathbf{T}(p, n)$ is contractible.

Every closed curve in $\mathbf{D}(\mathscr{G})$ is homotopic in $\mathbf{D}(\mathscr{G})$ to a closed curve in $\mathbf{D}_{0}(\mathscr{G})$. The ball $\left\{t \in \mathbb{C}^{d} ;|t|<e^{-2 \pi}\right\}$ is contained in $\mathbf{D}(\mathscr{G})$. Thus starting with a curve in $\mathbf{D}(\mathscr{G})$, we homotope it first to a curve in $\mathbf{D}_{0}(\mathscr{G})$, then to a curve in $\left\{t \in \mathbf{D}_{0}(\mathscr{G}) ;|t|<e^{-2 \pi}\right\}$, and finally we contract it to a point in $\left\{t \in \mathbb{C}^{d} ;|t|<\right.$ $\left.e^{-2 \pi}\right\}$. Thus $\mathbf{D}(\mathscr{G})$ is simply connected. Using Fenchel-Nielsen coordinates for $\mathbf{T}(p, n)$ one can show that $\mathbf{D}(\mathscr{G})$ is a cell and a domain of holomorphy. This result will appear in a paper of Earle-Kra-Marden [EKM]. 
9.9. We examine two important

Examples. (1) The graph $\mathscr{G}$ of Figure 10 has the following automorphisms:

(i) $\sigma\left(a_{2}\right)=a_{2}, \sigma\left(a_{1}\right)=a_{1}^{\varepsilon_{1}}, \sigma\left(a_{3}\right)=a_{3}^{\varepsilon_{3}}$,

(ii) $\sigma\left(a_{2}\right)=a_{2}^{-1}, \sigma\left(a_{1}\right)=a_{3}^{\varepsilon_{1}}, \sigma\left(a_{3}\right)=a_{1}^{\varepsilon_{3}}, \varepsilon_{1}= \pm 1, \varepsilon_{3}= \pm 1$.

The induced automorphisms of $\mathbb{C}^{3}$ send $\left(t_{1}, t_{2}, t_{3}\right)$ to

$$
\begin{array}{rr}
\left(t_{1}, t_{2}, t_{3}\right) & \text { in case (i) when } \varepsilon_{1} \varepsilon_{3}=+1 \text {, and to } \\
\left(t_{1},-t_{2}, t_{3}\right) & \text { when } \varepsilon_{1} \varepsilon_{3}=-1, \\
\left(t_{3}, t_{2}, t_{1}\right) & \text { in case (ii) when } \varepsilon_{1} \varepsilon_{3}=+1 \text {, and to } \\
\left(t_{3},-t_{2}, t_{1}\right) & \text { when } \varepsilon_{1} \varepsilon_{3}=-1 .
\end{array}
$$

Note that case (i) with $\varepsilon_{1}=-1=\varepsilon_{3}$ corresponds to the hyperelliptic involution (which acts trivially on $\mathbf{T}(2,0)$ ). We conclude that Aut $\mathscr{G} \cong D_{4}$, the 4-dihedral group $\left(\left|D_{4}\right|=8\right)$. The image of Aut $\mathscr{G}$ in Aut $D(\mathscr{G})$ is $\mathbb{Z}_{2} \oplus \mathbb{Z}_{2}$ (the kernel of the map described by Corollary 2 to Theorem 3.8 is $\mathbb{Z}_{2}$ ).

(2) The automorphism group of the graph $\mathscr{G}$ of Figure 11 has a normal subgroup of index 2 that fixes each vertex. This subgroup is the permutation group on three letters. It acts by permutation on $\mathbb{C}^{3}$. An extra element $\sigma$ of Aut $\mathscr{G}$ may be described by $\sigma\left(a_{j}\right)=a_{j}^{-1}, j=1,2,3$. It acts trivially on $\mathbb{C}^{3}$ and corresponds to the hyperelliptic involution. In this case Aut $\mathscr{G} \cong \mathscr{S}_{3} \oplus \mathbb{Z}_{2}$ (a group of order 12) maps onto a subgroup of $\operatorname{Aut} \mathbf{D}(\mathscr{G})$ isomorphic to $\mathscr{F}_{3}$ with kernel $\mathbb{Z}_{2}$.

Lemma. Let $\sigma \in$ Aut $\mathscr{G}$ with $\sigma^{*}=1$ and $\sigma \neq 1$. Then $\mathscr{G}$ is of type $(0,4)$, $(1,1),(1,2)$ or $(2,0)$. Further, in these exceptional cases $\left\{\sigma \in\right.$ Aut $\mathscr{G} ; \sigma^{*}=$ 1) is isomorphic to $\mathbb{Z}_{2} \oplus \mathbb{Z}_{2}$ for graphs of type $(0,4)$, and to $\mathbb{Z}_{2}$ for graphs of type $(1,1),(1,2)$ or $(2,0)$. These exceptional groups act as groups of conformal automorphisms that preserve each partition curve, on each Riemann surface $S_{t}$, $t \in \mathbf{D}(\mathscr{G})$.

Proof. The case $d=1$ has already been treated; so assume that $d>1$. If $\sigma^{*}=$ 1 , then $\sigma$ preserves each edge (it can send an edge to its inverse). If $\sigma\left(a_{j}\right)=a_{j}$, for $j=1, \ldots, d$, then $\sigma\left(S^{k}\right)=S^{k}$ for each vertex $k=1, \ldots, v$. In this case $\sigma$ can only permute a number of pairs of phantom edges corresponding to spherical ends. Let $a_{j}$ have two phantom edges emanating from one of its vertices. Assume that these phantom edges are permuted by $\sigma$. Consider the second vertex of $a_{j}$. Either two phantom edges emanate from it (thus we are in the $(0,4)$ case) or at least one edge emanates from it; it must be fixed by $\sigma$. It follows that $\sigma^{*}$ changes the sign of the $j$ th component of $t$.

The remaining possibility is that $\sigma\left(a_{j}\right)=a_{j}^{-1}$ for at least one $j$. If $a_{j}$ corresponds to an elliptic end, then the vertex of $a_{j}$ has another edge $a_{i}$, $i \neq j$, emanating from it (if it were a phantom edge, then we would be in the $(1,1)$ case). This edge $a_{i}$ is fixed by $\sigma$. If $a_{i}$ determines a spherical end, then we are in the case given by the first graph of type $(1,2)$ in Figure 6 . Thus 
the automorphism $\sigma$ must map $a_{1}$ to $a_{1}^{-1}$, fix $a_{2}$ and interchange $a_{3}$ and $a_{4}$. This automorphism of $\mathscr{G}$ arises from the hyperelliptic involution on the surface of type $(1,2)$. We leave the verification of this claim to the reader. If $a_{i}$ does not determine a spherical end, the second vertex of $a_{i}$ is joined to itself by an edge (otherwise $\sigma^{*}$ would change the sign of the $i$ th component of $t$ ). It follows that we are in the $(2,0)$ case of Figure 10 and $\sigma$ is the automorphism described by Example 1(i), with $\varepsilon_{1}=-1=\varepsilon_{3}$. If $a_{j}$ does not correspond to an elliptic end, then as above, it can be seen that we are in the siutation described by the second graph of type $(1,2)$ in Figure 6 or by the graph of type $(2,0)$ in Figure 11. The automorphism $\sigma$ is induced by the hyperelliptic involution on the surface.

Let $\mathscr{G}$ be an admissible graph of type $(p, n)$. We shall say that $\mathscr{G}$ is exceptional if for every torsion free terminal b-group $\Gamma$ of graph type $\mathscr{G}$, $N(\Gamma)$ is a proper extension of $\Gamma$.

Theorem. (a) The graph $\mathscr{G}$ is exceptional if and only if it is of type $(p, n)=$ $(0,3),(0,4),(1,1),(1,2)$ or $(2,0)$.

(b) If $\Gamma$ is a generic exceptional group of graph type $\mathscr{G}$, then

$$
N(\Gamma) / \Gamma \cong \begin{cases}\mathscr{S}_{3} \oplus \mathbb{Z}_{2} & \text { if }(p, n)=(0,3) \\ \mathbb{Z}_{2} \oplus \mathbb{Z}_{2} & \text { if }(p, n)=(0,4), \\ \mathbb{Z}_{2} & \text { if }(p, n)=(1,1),(1,2) \text { or }(2,0) .\end{cases}
$$

Proof. By a generic property, we mean one valid in a dense open subset of $\mathbf{D}_{0}(\mathscr{G})$. The results of Greenberg $[G]$ show that the theorem is a consequence of the lemma.

9.10. We have seen that every $\sigma \in$ Aut $\mathscr{G}$ with $\sigma^{*}=1$ arises from a conformal self-map of every surface $S_{t}, t \in \mathbf{D}(\mathscr{G})$. Hence such $\sigma$ are in the image of $g$. Let $\sigma \in$ Aut $\mathscr{G}$ with $\sigma^{*} \neq 1$. The automorphism $\sigma^{*}$ of $\mathbf{D}_{0}(\mathscr{G})$ lifts to $\mathbf{T}(p, n)$. By Ruyden's theorem [Ro], see also Earle-Kra [EK1], every automorphism of $\mathbf{T}(p, n)$ arises from an element of the modular $\operatorname{group} \operatorname{Mod}(p, n)$. This element of $\operatorname{Mod}(p, n)$ is induced by a self-map of $S$ that preserves the partition $\Sigma$ (hence an element of $\operatorname{Mod} \Gamma$ ) and projects to $\sigma$ under the map $g$. Thus we have reproven the surjectivity of $g$ of (9.1.1) and (9.6.1).

9.11. We have seen that for all $t \in \mathbf{D}(\mathscr{G})$ and all $\sigma \in$ Aut $\mathscr{G}, S_{t}$ is conformally equivalent to $S_{\sigma^{*}(t)}$ via a conformal map $h$ that preserves the partition $\Sigma$. Conversely, given a conformal map $h: S_{t^{(1)}} \rightarrow S_{t^{(2)}}$ for $t^{(1)}, t^{(2)} \in \mathbf{D}(\mathscr{G})$ with the property that $h$ preserves the partition $\Sigma$ (that is, for all $a \in \Sigma, h(a)$ is freely homotopic to $a$ ), then there exists a $\sigma \in$ Aut $\mathscr{G}$ so that $t^{(2)}=\sigma^{*}\left(t^{(1)}\right)$. We see, in particular, that in this case for $j=1, \ldots, d$, we have $t_{j}^{(2)}=(-1)^{\varepsilon_{j}} t_{\sigma_{2}^{-1}(j)}^{(1)}$ with $\varepsilon_{j}= \pm 1$, which implies $\left|t^{(2)}\right|=\left|t^{(1)}\right|$. 


\section{FoRgETFUL MAPS}

The deformation space $\mathbf{D}(\mathscr{G})$ fibers over lower dimensional deformation spaces. The projection maps for these fibrations are induced by the usual projections of $\mathbb{C}^{d}$ onto lower dimensional linear subspaces. This section begins a study of these fiber spaces.

10.1. Let $\mathscr{G}$ be an admissible graph of type $(p, n)$ and $\mathscr{G}^{\prime}$ a subgraph of $\mathscr{G}$ obtained by breaking a number of edges of $\mathscr{G}$. Let $a_{1}, \ldots, a_{d}$ be the edges of $\mathscr{G}$. Let $J$ be a subset of $\{1, \ldots, d\}$. Let us assume that $\mathscr{G}^{\prime}$ is obtained by breaking the edges $a_{j}$ with $j \in J$. Let

$$
\rho_{J}=\rho: \mathbf{D}(\mathscr{G}) \rightarrow \mathbf{D}\left(\mathscr{G}^{\prime}\right)
$$

be the canonical forgetful map (for $t \in \mathbf{D}(\mathscr{G})$, ignore the coordinates $t_{j}$ with $j \in J)$, and

$$
s=s_{J}: \mathbf{D}\left(\mathscr{G}^{\prime}\right) \rightarrow \mathbf{D}(\mathscr{G})
$$

the right inverse to the forgetful map (for $t \in \mathbf{D}\left(\mathscr{G}^{\prime}\right)$, insert a zero in the $j$ th coordinate for each $j \in J$ ). Then both $\rho$ and $s$ are holomorphic, $\rho \circ s=I$ (hence $\rho$ is surjective and $s$ is injective), and $\rho\left(\mathbf{D}_{0}(\mathscr{G})\right)=\mathbf{D}_{0}\left(\mathscr{G}^{\prime}\right)$.

10.2. Let $S_{J}$ be the surface (with partition) obtained by shrinking each curve $a_{j}, j \in J$, to a node. Let $\mathbf{T}_{J}(p, n)$ be the product of the Teichmüller spaces of the parts of $S_{J}$. As a result of Theorem 9.4, $\mathbf{T}_{J}(p, n)$ is a holomorphic universal covering space of $\mathbf{D}_{0}\left(\mathscr{G}^{\prime}\right)$, and there is a natural forgetful map $\mathbf{T}(p, n) \rightarrow \mathbf{T}_{J}(p, n)$ that covers $\rho: \mathbf{D}_{0}(\mathscr{G}) \rightarrow \mathbf{D}_{0}\left(\mathscr{G}^{\prime}\right)$.

10.3. Every complex manifold has two natural metrics on it: the Carathéodory metric $d_{c}$ and Kobayashi metric $d_{k}$ (see Kobayashi [Ko, Chapter IV]). In addition, the Teichmüller spaces $T(p, n)$ carry the Teichmüller metric $d_{T}$. It is well known that in general $d_{c} \leq d_{k}$; and as a consequence of a fundamental theorem of Royden [Ro] (see also [EK1]), $d_{c} \leq d_{k}=d_{T}$ on $\mathbf{T}(p, n)$. For simply connected domains in $\mathbb{C}$ with at least two boundary points, $d_{c}=d_{k}=$ Poincare metric $d_{p}$. If $d(\mathscr{G})=1$, then $\mathbf{D}(\mathscr{G})$ is biholomorphic to the unit disc. It follows that, in this case, the Carathéodory and Kobayashi metrics on $\mathbf{D}(\mathscr{G})$ coincide with the Poincare metric. As a consequence of the fact that holomorphic maps do not increase either Carathéodory or Kobayashi distance and the fact that $\rho \circ s=I$, the map $s$ is an isometry in both the Caratheodory and Kobayashi metrics, provided $\mathbf{D}\left(\mathscr{G}^{\prime}\right)$ has positive dimension. In particular, these invariant metrics on $\mathbf{D}(\mathscr{G})$ restrict to the Poincare metric on each coordinate plane $\left\{t \in \mathbf{D}(\mathscr{G}) ; t_{j}=0\right.$ for $\left.j \neq k\right\}, k=1, \ldots, d$.

10.4. Let $\mathbf{D}_{1}=\mathbf{D}(\mathscr{G})$ for $\mathscr{G}$ of type $(0,4)$ and $\mathbf{D}_{2}=\mathbf{D}(\mathscr{G})$ for $\mathscr{G}$ of type $(1,1)$. Then, we have shown (recall $(0.1)$ )

$$
\Delta_{e^{-\pi}} \subset \mathbf{D}_{1} \subset \Delta_{e^{-\pi / 2}}, \quad \Delta_{e^{-2 \pi}} \subset \mathbf{D}_{2} \subset \Delta_{e^{-\pi}} \quad \text { and } \quad \mathbf{D}_{2}=\left\{t^{2} ; t \in \mathbf{D}_{1}\right\} .
$$

For an arbitrary graph $\mathscr{G}$,

$$
\left(\Delta_{e^{-2 \pi}}\right)^{d} \subset \mathbf{D}(\mathscr{G}) \subset \mathbf{D}^{(1)} \times \cdots \times \mathbf{D}^{(d)},
$$


where $\mathbf{D}^{(j)}=\mathbf{D}_{1}$ if the edge $a_{j}$ corresponds to a four times punctured sphere, and $=\mathbf{D}_{2}$ if the edge $a_{j}$ corresponds to an elliptic end.

The above relations yield estimates for invariant metrics on the deformation spaces. They also prove Theorem $3(a)$.

\section{Metrics ON SURfaCes AND THeir TeICHMÜLLER SPACES}

We use estimates on the Poincaré metric on Riemann surfaces to obtain rough estimates for lengths of geodesics in a partition set.

11.1. For a hyperbolic domain $\mathbf{D}$ in $\hat{\mathbb{C}}$, we let $\lambda_{\mathbf{D}}(z)|d z|$ denotes its Poincaré metric of constant negative curvature -1 . The same notation will be employed on arbitrary Riemann surfaces, with the understanding that $z$ is a local coordinate on the surface.

11.2. We are interested in describing the behavior of partition curves as our parameters approach certain distinguished boundary points of $\mathbf{T}(p, n)$. We start by computing the Poincare metric $\lambda_{\mathbf{D}}(\zeta)|d \zeta|$ for the strip domain

$$
\mathbf{D}=\mathbf{D}_{a, k}=\{\zeta \in \mathbb{C} ; a<\operatorname{Im} \zeta<a+\pi k\} ;
$$

here $a \in \mathbb{R}$ and $k \in \mathbb{R}^{+}$. For the constant curvature -1 normalization,

$$
\lambda_{\mathbf{D}}(\zeta)=\left[k \sin \left(\frac{\operatorname{Im} \zeta-a}{k}\right)\right]^{-1} .
$$

The domain $\mathbf{D}$ is invariant under the group generated by the translation $A(\zeta)=$ $\zeta+2$. The factor space $\mathbf{D} /\langle A\rangle$ is the annulus

$$
\mathscr{A}=\mathscr{A}_{k}=\left\{z \in \mathbb{C} ; e^{-\pi^{2} k}<|z|<1\right\}
$$

(here $z=e^{\pi i(\zeta-i a)}$ ). The Poincare metric on this annulus is

$$
\lambda_{\mathscr{A}}(z)=\left[\pi k|z| \sin \left(-\frac{\log |z|}{\pi k}\right)\right]^{-1} .
$$

We note that $\lim _{k \rightarrow \infty} \lambda_{\mathscr{S}_{k}}(z)=[-|z| \log |z|]^{-1}$, which is the metric for the punctured unit disc.

Remark. For the normalization used in $\S 2.2$,

$$
\mathscr{A}=\mathscr{A}_{t}=\left\{z \in \mathbb{C} ;|t| e^{\pi / 2}<|z|<e^{-\pi / 2}\right\}, \quad|t|<e^{-\pi},
$$

we have

$$
\lambda_{\mathscr{A}}(z)=\left[-\left(\frac{\log |t|}{\pi}+1\right)|z| \sin \left(\frac{\pi(\log |z| / \pi+1 / 2)}{\log |t| / \pi+1}\right)\right]^{-1} .
$$

11.3. Consider the AFP construction of $\S 6.1$. The invariant component $\Delta$ of $\Gamma$ is trapped between two strips:

$$
\mathbf{D}_{0}=\mathbf{D}_{1 / 2,(\operatorname{Im} \alpha-1) / \pi} \subset \Delta \subset \mathbf{D}_{0, \operatorname{Im} \alpha / \pi}=\mathbf{D}_{1}
$$


(the second inclusion is valid for all $\alpha \in \mathbf{T}(0,4)$; for the first, we must assume that $\operatorname{Im} \alpha>1)$. Let $\tilde{a}$ be the geodesic on $\Delta / \Gamma$ corresponding to the Möbius transformation $A \in \Gamma$. Let $l(\alpha)$ be the length of the geodesic $\tilde{a}$ on the Riemann surface corresponding to the parameter $\alpha$. Then

$$
l(\alpha) \leq \int_{c(y)} \lambda_{\Delta}(\zeta)|d \zeta| \leq \int_{c(y)} \lambda_{\mathbf{D}_{0}}(\zeta)|d \zeta|,
$$

where $c(y)$ is the path $c(y)(s)=s+i y, 0 \leq s \leq 2$, and $y$ is arbitrary (but fixed) with $\frac{1}{2}<y<\operatorname{Im} \alpha-\frac{1}{2}$. Choosing $y$ to be $\frac{1}{2} \operatorname{Im} \alpha$, we see that $l(\alpha) \leq 2 \pi /(\operatorname{Im} \alpha-1)$, for all $\alpha \in \mathbf{T}(0,4)$ with $\operatorname{Im} \alpha>1$. In particular, $\lim _{\operatorname{Im} \alpha \rightarrow \infty} l(\alpha)=0$. To obtain an estimate in the opposite direction let $\tilde{\tilde{a}}$ be the lift to $\Delta$ of the geodesic freely homotopic to $\tilde{a}$ on $\Delta / \Gamma$. Then $\tilde{\tilde{a}}$ is a curve from a point $z_{0} \in \Delta$ to $z_{0}+2$, and

$$
l(\alpha)=\int_{\tilde{\tilde{a}}} \lambda_{\Delta}(\zeta)|d \zeta| \geq \int_{\hat{\tilde{a}}} \lambda_{\mathbf{D}_{1}}(\zeta)|d \zeta| \geq \frac{2 \pi}{\operatorname{Im} \alpha}, \quad \text { for all } \alpha \in \mathbf{T}(0,4) .
$$

We have shown that if $c:[0,1) \rightarrow \mathbf{T}(0,4)$ is a path in $\mathbf{T}(0,4)$, then (for the horocyclic coordinate $\alpha=c(s)$ )

$$
\lim _{s \rightarrow 1} l(c(s))=0 \Leftrightarrow \lim _{s \rightarrow 1} \operatorname{Im} c(s)=\infty .
$$

We observed that $\mathbf{T}(0,4) /(2 \mathbb{Z}) \cong \mathbf{D}_{0}(\mathscr{G})$, where $\mathscr{G}$ is the (unique) graph of type $(0,4)$. The coordinate $t$ on $\mathbf{D}(\mathscr{G})$ is related to the coordinates $\alpha$ on $\mathbf{T}(0,4)$ by $t=e^{\pi i \alpha}$. The length $l(t)$ of the curve $\tilde{a}$ on the surface corresponding to $t$ is a well-defined function on $\mathbf{D}(\mathscr{G})$. We have obtained the estimate

$$
\frac{-2 \pi^{2}}{\log |t|} \leq l(t) \leq \frac{-2 \pi^{2}}{\log |t|+\pi}
$$

(the first inequality is valid for all $t \in \mathbf{D}(\mathscr{G})$, the second only for $|t|<e^{-\pi}$ ).

11.4. Consider the general AFP construction for terminal $b$-groups. Assume that $\Gamma$ is the amalgamated free product of the groups $\Gamma_{1}$ and $\Gamma_{2}$ across the cyclic subgroup generated by $A=\left[\begin{array}{cc}-1 & -2 \\ 0 & -1\end{array}\right]$. Assume that our construction is tame and corresponds to the parameter $\alpha$ with $\operatorname{Im} \alpha>1$. It involves no loss of generality to assume that $\Gamma_{i} \supset F_{i}$ for $i=1,2$ (where the $F_{i}$ are defined by $\S 6.1)$. In this case $\alpha=f\left(B_{2}\right)$ and there exist real numbers $c_{1}, c_{2}$ with $c_{2}>c_{1}>0$ so that the regions $\left\{\zeta \in \mathbb{C} ; \operatorname{Im} \zeta>c_{1}\right\}$ and $\left\{\zeta \in \mathbb{C} ; \operatorname{Im} \zeta<c_{2}\right\}$ are precisely invariant under $\langle A\rangle$ in $\Gamma_{1}$ and $\Gamma_{2}$, respectively. It follows that the invariant component $\Delta$ of $\Gamma$ contains the strip $\left\{\zeta \in \mathbb{C} ; c_{1}<\operatorname{Im} \zeta<c_{2}\right\}$ and is contained in the strip $\{\zeta \in \mathbb{C} ; 0<\operatorname{Im} \zeta<\operatorname{Im} \alpha\}$. Let $l$ be the length of the geodesic on $\Delta / \Gamma$ determined by the element $A \in \Gamma$. It follows that $2 \pi / \operatorname{Im} \alpha \leq l \leq 2 \pi /\left(c_{2}-c_{1}\right)$. As before, the first inequality is valid for all $\alpha$. Thus $\operatorname{Im} \alpha \rightarrow \infty$ as $l \rightarrow 0$.

If the parameters for both $\Gamma_{1}$ and $\Gamma_{2}$ vary in compact subsets of their respective deformation spaces, then both $c_{1}$ and $c_{2}$ can be chosen independently 
of the groups $\Gamma_{1}$ and $\Gamma_{2}$ and we see in this case $c_{2}-c_{1}$ is of the form $\operatorname{Im} \alpha-c$ for some $c \in \mathbb{R}, c>0$. It follows that $l \rightarrow 0$ as $\operatorname{Im} \alpha \rightarrow \infty$.

Assume that both $\Gamma_{1}$ and $\Gamma_{2}$ have been constructed using only tame plumbings with gluing parameters $t$ of norm $|t|<e^{-2 \pi}$. It follows that we can choose in this case $c_{1}=1$ and $c_{2}=\operatorname{Im} \alpha-1$. We obtain the universal estimate for $t \in \mathbf{D}(\mathscr{G}),|t|<e^{-2 \pi}$,

$$
\frac{-2 \pi^{2}}{\log |t|} \leq l(t) \leq \frac{-2 \pi^{2}}{\log |t|+2 \pi} .
$$

11.5. For the HNN construction of $\S 6.3$, the invariant component $\Delta$ is contained in a strip of width $\operatorname{Im} \tau$ and contains a strip of width $\operatorname{Im} \tau-2$ (for $\tau \in \mathbb{C}$ with $\operatorname{Im} \tau>2)$. Thus the appropriate bounds on the length $l$ of the geodesic corresponding to the central curve on $S$ are given by (11.4.1). The same estimate is valid for the general HNN construction of the surface $S_{t}$ as long as $|t|<e^{-2 \pi}$.

11.6. Let $\mathscr{G}$, as before, be an admissible graph of type $(p, n)$ with edges $a_{1}, \ldots, a_{d}$. We define a mapping $l: \mathbf{D}(\mathscr{G}) \rightarrow \mathbb{R}^{d}$ by

$$
l(t)=l\left(t_{1}, \ldots, t_{d}\right)=\left(l_{1}(t), \ldots, l_{d}(t)\right),
$$

where $l_{j}(t)$ is the hyperbolic length on $S_{t}$ of the geodesic freely homotopic to $a_{j}$. Note that

$$
l_{j}(t)=0 \Leftrightarrow t_{j}=0, \quad j=1, \ldots, d .
$$

We observe that $l$ is well defined on $\mathbf{D}(\mathscr{G})$ and it is continuous on $\mathbf{D}_{0}(\mathscr{G})$ (since all geodesic length functions are continuous on $\mathbf{T}(p, n)$, and these particular length functions are invariant under $\left.(2 \mathbb{Z})^{d}\right)$. Continuity on $\mathbf{D}(\mathscr{G})$ will be studied in [EKM]. We observe that as a consequence of $\S \S 11.4,11.5$ and 9.5, we have the following for $t^{(n)} \in \mathbf{D}(\mathscr{G})$ with $t^{(n)}=\left(t_{1}^{(n)}, \ldots, t_{d}^{(n)}\right)$. If $\lim _{n \rightarrow \infty} t^{(n)}=t$ with $t=\left(t_{1}, \ldots, t_{d}\right) \in \mathbf{D}(\mathscr{G})$, then $\lim _{n \rightarrow \infty} l_{j}\left(t^{(n)}\right)=0$ if and only if $t_{j}=0$.

We have completed the proof of Theorem 3 of the Introduction.

11.7. The following result is a well-known application of the collar lemma.

Proposition. Let $S$ be a Riemann surface (possibly with nodes) of finite hyperbolic type. Let $\Sigma$ be a maximal partition on $S$. Assume that every curve in $\Sigma$ is freely homotopic to a geodesic of length $\leq 2 \operatorname{arcsinh} 1$. Let $h: S \rightarrow S$ be a conformal automorphism. Then for every $a \in \Sigma, h(a)$ is freely homotopic to a curve in $\Sigma$.

Proof. Without loss of generality, every curve in $\Sigma$ is either a geodesic or a node. Since $h$ is conformal, it is an isometry in the Poincare metric on $S$. Thus $a$ and $h(a)$ have the same length for all $a \in \Sigma$; in particular, $h(a)$ is a node if only if $a$ is a node. Thus $h$ permutes the components of the complement of the nodes on $S$. Let $a$ be a curve in $\Sigma$ with length $l(a)>0$. 
If $h(a)$ is not in $\Sigma$, then it must intersect a curve, say $b$ (not a node), in $\Sigma$ because each part of $S-\Sigma$ is a thrice punctured sphere. By the Keen-Halpern collar lemma in its sharp form (Matelski [Ms] or Buser [Bu])

$$
\sinh \frac{l(b)}{2} \sinh \frac{l(h(a))}{2}>1 \text {. }
$$

This contradicts the hypothesis that the length of all the curves in $\Sigma$ (hence also in $h(\Sigma)$ ) are short.

Remark. Note that $2 \operatorname{arcsinh} 1=2 \log (1+\sqrt{2})=1.762747 \ldots$.

11.8. As an application of the above proposition and our estimates on the lengths of geodesics, we prove the following

Theorem. There exists a universal constant

$$
\varepsilon>\varepsilon_{1}=e^{-2 \pi} e^{-\pi^{2} / \operatorname{arcsinh} 1}=2.5587 \ldots \times 10^{-3}
$$

such that for all admissible graphs $\mathscr{G}$ and all $t^{(1)}, t^{(2)}$ in $\mathbf{D}(\mathscr{G})$ with $\left|t^{(j)}\right|<\varepsilon$, $j=1,2$, we have $S_{t^{(1)}} \cong S_{t^{(2)}}$ (conformal equivalence) if and only if there is $a$ $\sigma \in$ Aut $\mathscr{G}$ such that $\sigma^{*}\left(t^{(1)}\right)=t^{(2)}$.

Proof. We have seen $(\S 9.10)$ that $t^{(2)}=\sigma^{*}\left(t^{(1)}\right)$ for some $\sigma \in$ Aut $\mathscr{G}$ implies that $S_{t^{(2)}}$ is conformally equivalent to $S_{t^{(1)}}$. Conversely, let $h: S_{t^{(1)}} \rightarrow S_{t^{(2)}}$ be a conformal equivalence. It suffices to show that $h$ preserves the partition (see $\S 9.11)$. Clearly $h$ sends nodes to nodes and preserves lengths of partition curves (which may be assumed to be geodesics). Thus if $\Sigma$ is a partition on $S_{t^{(1)}}$, then $\Sigma$ and $h(\Sigma)$ are partitions on $S_{t^{(2)}}$. The lengths of the curves in these partitions are bounded from above by $-2 \pi^{2} /(\log |t|+2 \pi) \leq 2 \operatorname{arcsinh} 1$ (here $t$ is a component of $t^{(2)}$ or $t^{(1)}$ ) as long as $\left|t^{(j)}\right| \leq \varepsilon$ for $j=1,2$. The two partitions coincide by Proposition 11.7.

\section{Appendix I: Calculations IN $\operatorname{PSL}(2, \mathbb{C})$ and $\operatorname{SL}(2, \mathbb{C})$}

We collect in this section various useful calculations with Möbius transformations that were needed in our work. Throughout "triangle group" means "torsion free triangle group". Most of the results are invariant forms of calculations previously encountered for special cases (see, for example, $\S \S 1.2,6.1$ and 6.3). We omit the details of the proofs.

12.1. Proposition. Let $a, b, c$ be three distinct points in $\hat{\mathbb{C}}$. There exists $a$ unique triangle group $F(a, b, c)$ with canonical generators $(A, B)$ such that $f(A)=a, f(B)=b$ and $f(A \circ B)=c$ and a unique isomorphism $\theta$ with $\theta(A)=\left[\begin{array}{cc}-1 & -2 \\ 0 & -1\end{array}\right], \theta(B)=\left[\begin{array}{cc}-1 & 0 \\ 2 & -1\end{array}\right]$. The Möbius transformations $A, B, C=$ $B^{-1} \circ A^{-1}$ may be described as the unique elements of $\operatorname{SL}(2, \mathbb{C})$ with

$$
\operatorname{tr} A=\operatorname{tr} B=\operatorname{tr} C=-2, \quad A \circ B \circ C=I,
$$


$A, B$ and $C$ fix (only) $a, b$ and $c$, respectively. If we define $M(a, b, c)$ by

$$
M(a, b, c)=\left[\begin{array}{cc}
-1-\frac{2 a(b-c)}{(a-b)(a-c)} & \frac{2 a^{2}(b-c)}{(a-b)(a-c)} \\
-\frac{2(b-c)}{(a-b)(a-c)} & -1+\frac{2 a(b-c)}{(a-b)(a-c)}
\end{array}\right],
$$

then formulae for the generators of $F(a, b, c)$ are

$$
A=M(a, b, c), \quad B=M(b, c, a) \text { and } C=M(c, a, b) .
$$

Proof. Conjugate the group $F=F(\infty, 0,1)$ of $\S 1.2$ by $E=E(a, b, c) \in$ $\operatorname{PSL}(2, \mathbb{C})$ that satisfies

$$
E(a)=\infty, \quad E(b)=0, \quad E(c)=1 .
$$

Then $E F(a, b, c) E^{-1}=F(\infty, 0,1)$.

Notation. Whenever we write $F(a, b, c)$ we will mean the triangle group along with the canonical generators specified by the above formulae. Thus $F(\infty, 0,1), F(\infty, 1,0)$ and $F(\infty,-1,0)$ denote the same group with the different sets of canonical generators.

Remarks. (1) A parabolic element $A \in \mathrm{SL}(2, \mathbb{C})$ with $\operatorname{tr} A=-2$ and $f(A)=$ $a \in \mathbb{C}$ can be written in "normal form" as

$$
\left[\begin{array}{cc}
-1-a \alpha & a^{2} \alpha \\
-\alpha & -1+a \alpha
\end{array}\right], \quad \alpha \in \mathbb{C}^{*}
$$

(alternately, we can write $1 /(A(z)-a)=1 /(z-a)+\alpha, z \in \hat{\mathbb{C}})$. We will call $\alpha$ the translation length of $A$.

(2) The case $a=\infty$ can be considered as the limiting situation of the general formula, if we regard $\alpha=0$ with $c=a^{2} \alpha \neq 0$ (thus, also $a \alpha=a^{2} \alpha / a=0$ ). We will follow such conventions in the sequel, whenever appropriate. (For $a=\infty,-a^{2} \alpha$ should be regarded as the translation length of $A$.)

(3) The element $A$ (defined by (12.1.1)) in the lemma has fixed point $a$ and translation length $2(b-c) /(a-b)(a-c)$, provided $a \neq \infty$; for $a=\infty$, the translation length of $A$ is $-2(b-c)$.

(4) Note that every parabolic element has a well-defined (unique) parabolic square root in $\operatorname{PSL}(2, \mathbb{C})$ and that $f(C)=A^{1 / 2}(f(B))$. (It hence also follows that $f(A)=B^{1 / 2}(f(C))$ and $f(B)=C^{1 / 2}(f(A))$.) Thus

$$
F(a, b, c)=F\left(f(A), f(B), A^{1 / 2}(f(B))\right),
$$

and the group with canonical generators $\left(A^{-1}, B^{-1}\right)$ is $F\left(a, b, A^{-1 / 2}(b)\right)$.

Corollary 1. For all distinct triples $a, b$ and $c$ in $\hat{\mathbb{C}}$ and all $E \in \operatorname{PSL}(2, \mathbb{C})$,

$$
E F(a, b, c) E^{-1}=F(E(a), E(b), E(c)) .
$$

Corollary 2. Let $F_{j}=F\left(a_{j}, b_{j}, c_{j}\right)$ be triangle groups with corresponding canonical generators $\left(A_{j}, B_{j}\right)$ for $j=1,2$. Let $E_{j}=E\left(a_{j}, b_{j}, c_{j}\right)$ (as defined by (12.1.2)). Then $F_{1}=F_{2}$ if and only if $T=E_{2}^{-1} \circ E_{1} \in \operatorname{PGL}(2, \mathbb{Z})$. Further, 
in case of equality, the punctures determined by $a_{2}, b_{2}$ lie on the same sides of horocircles determined by

$$
\left(A_{2}, B_{2}\right) \text { and }\left(E_{2} \circ E_{1}^{-1} \circ A_{1} \circ E_{1} \circ E_{2}^{-1}, E_{2} \circ E_{1}^{-1} \circ B_{1} \circ E_{1} \circ E_{2}^{-1}\right)
$$

if and only if $\operatorname{det} T=+1$.

Proof. Use the results of $\S 1.7$.

12.2. Lemma. Given a parabolic element $A \in \mathrm{SL}(2, \mathbb{C})$ with $\operatorname{tr} A=-2$ and given $b \in \hat{\mathbb{C}}$ with $b \neq f(A)$, there exists a unique parabolic $B \in \mathrm{SL}(2, \mathbb{C})$ such that $f(B)=b$ and $\operatorname{tr} B=-2=\operatorname{tr}(A \circ B)$.

Proof. Use the normal forms for $A$ and $B$ and consider two cases: $f(A)=\infty$ or $f(A) \in \mathbb{C}$.

Corollary 1. Given a parabolic element $A \in \mathrm{SL}(2, \mathbb{C})$ with $\operatorname{tr} A=-2$ and $f(A)=a$, and given $b \in \hat{\mathbb{C}}, b \neq a$, the unique triangle group with canonical generators $(A, B)$ with $f(B)=b$ is given by

$$
F\left(a, b, \frac{a(b-a) \alpha+2 b}{(b-a) \alpha+2}\right)=F\left(a, b, A^{1 / 2}(b)\right),
$$

where $\alpha$ is the translation length of $A$. (The first expression must be appropriately interpreted for $a=\infty$ or $b=\infty$ in accordance with Remark 2 of $\S 12.1$.)

Corollary 2. Let $A, B_{1}, B_{2} \in \operatorname{PSL}(2, \mathbb{C})$ with $\left(A, B_{j}\right), j=1,2$, canonical generators for the triangle group $F_{j}$. Then $B_{1}=B_{2} \Leftrightarrow f\left(B_{1}\right)=f\left(B_{2}\right)$.

Corollary 3. Let $F=F(a, b, c)$ be a triangle group with corresponding canonical generators $(A, B)$. Let $\tau \in \mathbb{C}, \tau \neq 0,1$. Then there exists a unique triangle group $F_{1}$ with canonical generators $\left(A, B_{1}\right)$, such that $\tau=\operatorname{cr}\left(f\left(B_{1}\right), a, b, c\right)$.

Proof. Solve for the fixed point $b_{1}$ of $B_{1}$ in $\tau=\operatorname{cr}\left(b_{1}, a, b, c\right)$ and use Corollary 1 with $b$ replaced by $b_{1}$. Then

$$
b_{1}=\frac{a(c-b) \tau+b(a-c)}{(c-b) \tau+(a-c)} .
$$

The translation length of $B_{1}$ is

$$
\beta_{1}=\frac{2(a(c-b) \tau+(a-c))^{2}}{(b-c)(c-a)(c-b)} .
$$

12.3. Lemma. Given two parabolic elements $A_{1}$ and $A_{2}$ in $\operatorname{SL}(2, \mathbb{C})$ with distinct fixed points and each with trace -2 , and given $\tau \in \mathbb{C}$, there exist precisely two $C \in \operatorname{SL}(2, \mathbb{C})$ with $C \circ A_{2} \circ C^{-1}=A_{1}$ and $\operatorname{tr} C=i \tau$.

Proof. The lemma is the invariant form of a result established in $\S 6.3$.

Remark. The two elements of $\operatorname{SL}(2, \mathbb{C})$ that satisfy the above conditions project to distinct elements of $\operatorname{PSL}(2, \mathbb{C})$ if and only if $\tau \neq 0$. 
12.4. Proposition. Let $(A, B)$ be canonical generators for a triangle group $F$. Let $\tau \in \mathbb{C}, \tau \neq 0,1$. There exists a unique $C \in \mathrm{SL}(2, \mathbb{C})$ such that

(i) $C \circ B \circ C^{-1}=A^{-1}$,

(ii) $\operatorname{tr} C=i \tau$, and

(iii) $\operatorname{cr}(C(f(A)), f(A), f(B), f(A \circ B))=\tau$.

Moreover, conditions (i) and (iii) yield a unique element $C$ of $\operatorname{PSL}(2, \mathbb{C}) ; a$ lift of $C$ to $\mathrm{SL}(2, \mathbb{C})$ can be chosen to satisfy (ii).

Proof. By conjugation we reduce to the case considered in $\S 6.3$.

12.5. We describe a formula for obtaining the Möbius transformation $C$. Solve for $b_{2}$ in $\tau=\operatorname{cr}\left(b_{2}, f(A), f(B), f(A \circ B)\right)$. Choose $B_{2} \in \mathrm{SL}(2, \mathbb{C})$ so that $f\left(B_{2}\right)=b_{2}, \operatorname{tr} B_{2}=-2=\operatorname{tr}\left(A \circ B_{2}\right)$. Choose $C \in \operatorname{PSL}(2, \mathbb{C})$ such that $C(f(A))=f\left(B_{2}\right), C(f(B))=f(A), C(f(A \circ B))=f\left(A \circ B_{2}\right)$. Then $C$ satisfies the conditions of the proposition.

Remark. Choose the invariant component $\Delta$ of $F$ so that $f(A)$ lies to the left of the horocircles determined by $A$ on $\Delta$. Assume that $\operatorname{Im} \tau \neq 0$. Then $\operatorname{Im} \tau>$ 0 if and only if $f(B)$ lies to the right of the horocircles determined by $A$ on $\Delta$. Similarly let $\left(A, B_{j}\right), j=1,2$, be canonical generators of the triangle group $F_{j}$. Assume that $\operatorname{Im} \tau \neq 0$, where $\tau=\operatorname{cr}\left(f\left(B_{2}\right), f(A), f\left(B_{1}\right), f\left(A \circ B_{1}\right)\right)$. Let $\Lambda_{j}$ be the limit set of $F_{j}$. Then $\Lambda_{j}$ is a circle. The two circles are tangent at $f(A)$. Let $D$ be the region between these circles. Then $\operatorname{Im} \tau>0$ if and only if $f\left(B_{1}\right)$ lies to the right of the horocircles determined by $A$ in $D$.

12.6. Proposition. Let $F_{j}=F\left(a_{j}, b_{j}, c_{j}\right)$ be a triangle group with corresponding canonical generators $\left(A_{j}, B_{j}\right), j=1,2$. Let $\tau \in \mathbb{C}, \tau \neq 0,1$. There exists a unique $C \in \operatorname{PSL}(2, \mathbb{C})$ with $C \circ A_{2} \circ C^{-1}=A_{1}$ and $\tau=\operatorname{cr}\left(C\left(b_{2}\right), a_{1}, b_{1}, c_{1}\right)$. Proof. Solve for $b_{3}$ in $\tau=\operatorname{cr}\left(b_{3}, a_{1}, b_{1}, c_{1}\right)$. Then $b_{3} \neq a_{1}$. Find $B_{3}=$ $\operatorname{SL}(2, \mathbb{C})$ with $f\left(B_{3}\right)=b_{3}, \operatorname{tr} B_{3}=-2=\operatorname{tr}\left(A_{1} \circ B_{3}\right)$. Let $c_{3}=f\left(A_{1} \circ B_{3}\right)$. Then find $C$ that satisfies $C\left(a_{2}\right)=a_{1}, C\left(b_{2}\right)=b_{3}, C\left(c_{2}\right)=c_{3}$.

Remark. Proposition 12.4 is a special case of Proposition 12.6 with $F_{1}=$ $F(a, b, c)$ and $F_{2}=F(b, a, c)$, where $a=f(A), b=f(B)$ and $c=$ $f\left(B^{-1} \circ A^{-1}\right)$. Note that $(A, B)$ are the canonical generators for $F_{1}$ and $\left(B^{-1}, A^{-1}\right)$ are the canonical generators for $F_{2}$.

\section{APPENDIX II. A COMPUTER PROGRAM FOR COMPUTING TORSION FREE TERMINAL $b$-GROUPS}

Let $\mathscr{G}$ be an admissible graph with a semicanonical ordering for its edges. Let $d=d(\mathscr{G})$. Let $t=\left(t_{1}, \ldots, t_{d}\right) \in \mathbb{C}^{d}$ with $0<\left|t_{j}\right|<1$ for $j=1, \ldots, d$. The construction algorithm described in $\S 7.5$ can be easily translated to a computer program that produces a group $\Gamma_{t}$ of Möbius transformations. This group is a $b$-group of graph type $\mathscr{G}$ if and only if $t \in \mathbf{D}_{0}(\mathscr{G})$. In such cases the program produces as output generators (as elements of $\mathrm{SL}(2, \mathbb{C})$ ) and relations 
for $\Gamma_{t}$, as well as the structure and modular subgroups of $\Gamma_{t}$. This program was implemented on MACSYMA to compute the examples in $\$ 7.5$. The program also produces the correct "boundary group" $\Gamma_{t}$ whenever $t \in \partial \mathbf{D}(\mathscr{G})$ and none of the coordinates of $t$ vanish. These boundary groups are studied in a sequel to this paper.

\section{APPENDIX III. INDEPENDENCE OF GLUING ON CHOICE OF ANNULI}

The following argument that the plumbing construction is independent of the choice of overlapping annuli is due to $\mathrm{S}$. Wolpert.

14.1. We introduce some convenient terminology. Let $\mathscr{A}$ be a closed connected set on a Riemann surface $S$. Assume that $S-\mathscr{A}$ has two components, one of which is a punctured disc and the other has a nonabelian fundamental group. We call the punctured discs the inside of $\mathscr{A}$ and the other the outside of $\mathscr{A}$.

14.2. For $j=1,2$, let $D^{j}$ be a punctured disc on the surface $S^{j}$ with boundary curve $\alpha_{j}$ contained in $S^{j}$. Let $z$ (respectively, $w$ ) be a local coordinate on the closure of $D^{1}\left(D^{2}\right)$ that vanishes at the puncture. We are allowing the possibility that $S^{1}=S^{2}$; in this case we assume that the closures of $D^{1}$ and $D^{2}$ are disjoint. Let $\mathscr{A}_{j}$ be an annulus whose closure is in $D^{j}$. Assume that the central curve on $\mathscr{A}_{j}$ is contractible to the puncture on $D^{j}$. Define

$$
S_{*}^{j}=S^{j}-\left\{\text { closure of inside of closure of } \mathscr{A}_{j}\right\} .
$$

Assume that there is a nonzero complex number $t$ such that for each $P \in \mathscr{A}_{1}$, there exists a unique $Q \in \mathscr{A}_{2}$ with $z(P) w(Q)=t$ and that the resulting map from $\mathscr{A}_{1}$ to $\mathscr{A}_{2}$ is surjective. Let $\sim^{*}$ denote the plumbing equivalence using the above annuli and the given value of the plumbing parameter and set

$$
S_{*}=S_{*}^{1} \cup S_{*}^{2} / \sim^{*} \text {. }
$$

Let $\mathscr{A}$ be the image of $\mathscr{A}_{1}$ in $S_{*}$. It is also the image of $\mathscr{A}_{2}$ in $S_{*}$.

14.3. We construct a surface $S_{* *}$ that depends only on $D^{1}, D^{2}$ and $t$ (and not on $\mathscr{A}_{1}$ or on $\mathscr{A}_{2}$ ). Let $\beta_{1}$ be the image in $S^{1}$ of $\alpha_{2} \subset S^{2}$ by the inversion $w \mapsto t / w$; that is,

$$
\beta_{1}=\left\{P \in S^{1} ; \exists Q \in \alpha_{2} \text { with } z(P) w(Q)=t\right\} .
$$

Similarly,

$$
\beta_{2}=\left\{Q \in S^{2} ; \exists P \in \alpha_{1} \text { with } z(P) w(Q)=t\right\} .
$$

For $j=1,2$, let

$$
\mathscr{A}_{j}^{*}=\left\{\text { inside of } \alpha_{j}\right\}-\left\{\text { closure of inside of } \beta_{j}\right\}
$$

and

$$
S_{* *}^{j}=S^{j}-\left\{\text { closure of inside of closure of } \mathscr{A}_{j}^{*}\right\}
$$




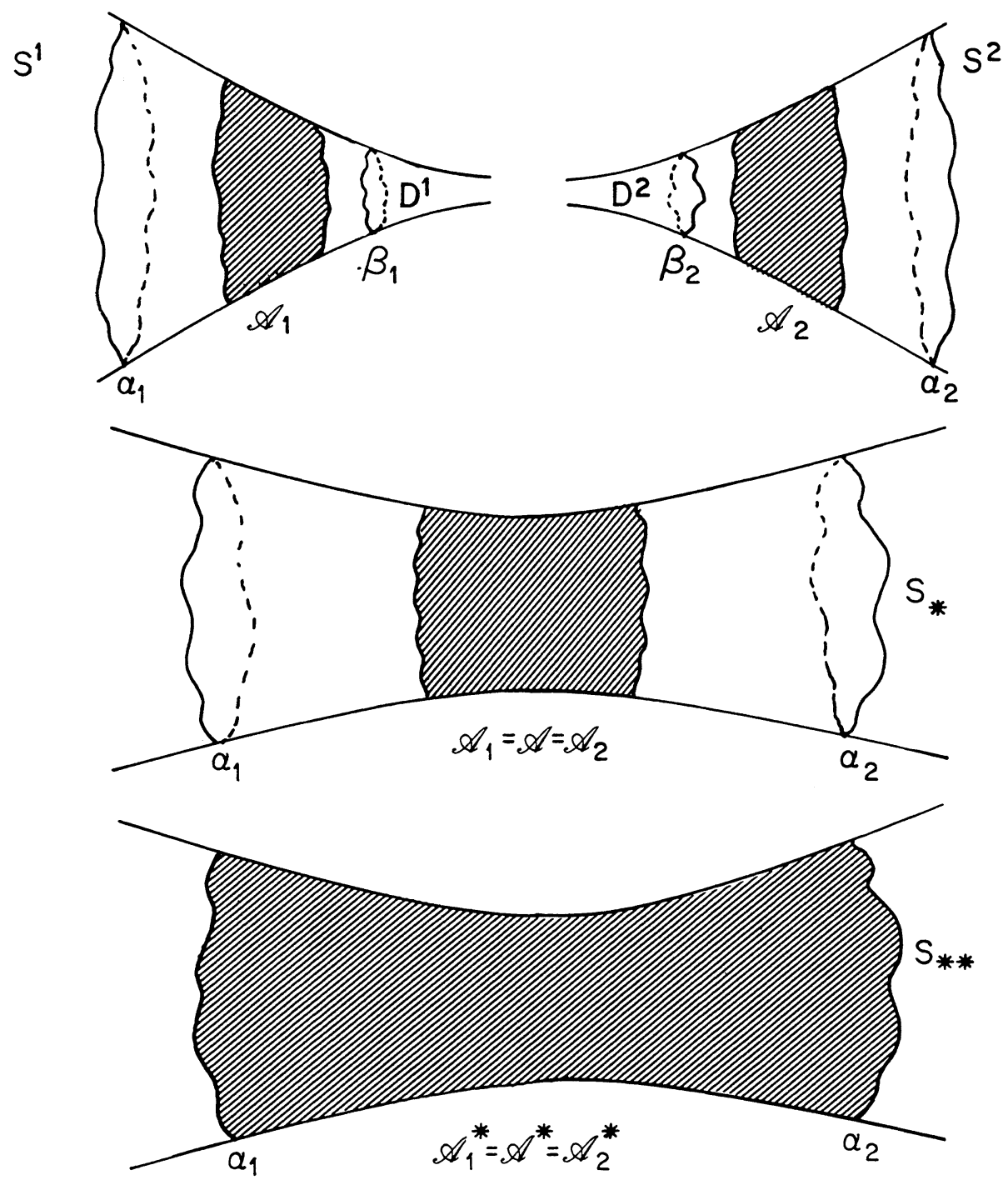

FIGURE 14. Gluing with different annuli.

Let $\sim^{* *}$ denote the plumbing equivalence using $\mathscr{A}_{1}^{*}, \mathscr{A}_{2}^{*}$ and $t$, and define

$$
S_{* *}=S_{* *}^{1} \cup S_{* *}^{2} / \sim^{* *} \text {. }
$$

The surface $S_{* *}$ is independent of the choice of $\mathscr{A}_{1}$ and $\mathscr{A}_{2}$. We claim that there exists a canonical biholomorphism (constructed in the next subsection) from $S_{* *}$ to $S_{*}$.

14.4. The relative positions of $\alpha_{1}, \beta_{1}, \beta_{2}, \alpha_{2}$ and the boundary curves of $\mathscr{A}_{1}$ and $\mathscr{A}_{2}$ are shown in Figure 14; that is, since $\alpha_{1}$ is outside $\mathscr{A}_{1}, \beta_{2}$ is inside $\mathscr{A}_{2}$ and hence also inside of $\alpha_{2}$ (similarly, $\beta_{1}$ is inside $\alpha_{1}$ ). We first extend 
the domain of the local coordinate $z$ on $S_{*}$. Let

$$
\tilde{z}(P)= \begin{cases}z(P), & P \in \mathscr{A} \text { or } P \in\left\{\text { annulus bounded by } \alpha_{1} \text { and } \mathscr{A}\right\}, \\ t / w(P), & P \in \mathscr{A} \text { or } P \in\left\{\text { annulus bounded by } \mathscr{A} \text { and } \alpha_{2}\right\} .\end{cases}
$$

The local coordinate $\tilde{z}$ is an analytic continuation of $z$. Define

$$
S_{*}^{\text {left }}=\text { connected component of }\left(S_{*}-\alpha_{2}\right) \text { containing } \mathscr{A},
$$

and observe that $S_{*}^{\text {left }}$ is canonically biholomorphic to $S_{* *}^{1}$ since $\tilde{z}$ allows us to extend the natural embedding of $S_{*}^{1}$ into $S_{*}$ to a natural embedding of $S_{* *}^{1}$ into $S_{*}$. We map $P \in S_{* *}^{1}-S_{*}^{1}$ to $Q \in S_{*}$ provided $z(P)=\tilde{z}(Q)$. Similarly, we define $\tilde{w}$ and $S_{*}^{\text {right }}$ and we obtain a natural embedding of $S_{*}^{\text {right }}$ (which is biholomorphic to $S_{* *}^{2}$ ) into $S_{*}$.

We have produced natural embeddings of $S_{* *}^{1}$ and $S_{* *}^{2}$ into $S_{*}$ and $P \in S_{* *}^{1}$ is identified with $Q \in S_{* *}^{2}$ by $\sim^{* *}$ if and only if $P$ and $Q$ map to the same point. This completes the proof that $S_{* *}$ is biholomorphically equivalent to the original surface $S_{*}$.

14.5. Up to now $z$ and $w$ were arbitrary coordinates on the punctured discs. If $z$ and $w$ are horocyclic coordinates, then the above arguments show that construction is also independent of the discs $D^{1}$ and $D^{2}$ (that is, the construction is independent of the branches of the $z$ and $w$ coordinates used).

\section{REFERENCES}

[A1] L. V. Ahlfors, The complex analytic structure of the space of closed Riemann surfaces, in Analytic Functions (R. Nevanlinna et. al., eds.), Princeton Univ. Press, Princeton, NJ, 1960, pp. 45-66.

[A2] _ The modular function and geometric properties of quasiconformal mappings, Proc. Minn. Conf. on Complex Analysis, Springer, 1965, pp. 296-300.

[AB] L. V. Ahlfors and L. Bers, Riemann's mapping theorem for variable metrics, Ann. of Math. (2) 72 (1960), 385-404.

[B1] L. Bers, Spaces of Riemann surfaces, Proc. Internat. Congr. Math. (Edinburgh, 1958), Cambridge, 1960, pp. 349-361.

[B2] - Simultaneous uniformization, Bull. Amer. Math. Soc. 66 (1960), 94-97.

[B3] _ Uniformization by Beltrami equations, Comm. Pure Appl. Math. 14 (1961), 215-228.

[B4] _ A non-standard integral equation with applications to quasiconformal mappings, Acta Math. 116 (1966), 113-134.

[B5] __ Spaces of Kleinian groups, in Several Complex Variables, Maryland 1970, Lecture Notes in Math., vol. 155, Springer, Berlin, 1970, pp. 9-34.

[B6] _ _ Fiber spaces over Teichmüller spaces, Acta Math. 130 (1973), 89-126.

[B7] _ $\ldots$, On spaces of Riemann surfaces with nodes, Bull. Amer. Math. Soc. 80 (1974), 12191222.

[B8] _ - Spaces of degenerating Riemann surfaces, in Discontinuous Groups and Riemann Surfaces, Ann. of Math. Stud., no. 79, Princeton Univ. Press, Princeton, NJ, 1974, pp. 43-59.

[B9] __ Deformations and moduli of Riemann surfaces with nodes and signatures, Math. Scand. 36 (1975), 12-16. 
[B10] __ An extremal problem for quasiconformal mappings and a theorem by Thurston, Acta Math. 141 (1978), 73-98.

[B11] _ Finite dimensional Teichmüller spaces and generalizations, Bull. Amer. Math. Soc. (N.S.) 5 (1981), 131-172.

[BG] L. Bers and L. Greenberg, Isomorphisms between Teichmüller spaces, in Advances in the Theory of Riemann Surfaces, Ann. of Math. Stud., no. 66, Princeton Univ. Press, Princeton, NJ, 1971, pp. 53-79.

[Bu] P. Buser, The collar theorem and examples, Manuscripta Math. 25 (1978), 349-357.

[DM] P. Deligne and D. Mumford, The irreducibility of the space of curves of a given genus, Inst. Hautes Études Sci. Publ. Math. 36 (1969), 75-109.

[E] C. J. Earle, Some intrinsic coordinates on Teichmüller spaces, Proc. Amer. Math. Soc. 83 (1981), 527-531.

[EK1] C. J. Earle and I. Kra, On holomorphic mappings between Teichmüller spaces, in Contributions to Analysis (L. V. Ahlfors et. al., eds.), Academic Press, New York, 1974, pp. 107-124.

[EK2] __, Half-canonical divisors on variable Riemann surfaces, J. Math. Kyoto Univ. 26 (1986), 39-64.

[EKM] C. J. Earle, I. Kra, and A. Marden, Convexity of moduli spaces (to appear).

[EM] C. J. Earle and A. Marden, Geometric complex coordinates for Teichmüller space (to appear).

[ES] C. J. Earle and P. L. Sipe, Families of Riemann surfaces over the punctured disk (to appear).

[F] J. D. Fay, Theta functions on Riemann surfaces, Lecture Notes in Math., vol. 362, SpringerVerlag, New York, 1973.

[G] L. Greenberg, Maximal Fuchsian groups, Bull. Amer. Math. Soc. 69 (1963), 569-573.

[H1] D. A. Hejhal, On Schottky and Teichmüller spaces, Adv. in Math. 15 (1975), 133-156.

[H2] _ Regular b-groups, degenerating Riemann surfaces, and spectral theory (to appear).

[Ko] S. Kobayashi, Hyperbolic manifolds and holomorphic mappings, Dekker, New York, 1970.

[K1] I. Kra, Deformations of Fuchsian groups. II, Duke Math. J. 38 (1971), 499-508.

[K2] _-, On spaces of Kleinian groups, Comment. Math. Helv. 47 (1972), 53-69.

[K3] _ On Teichmüller's theorem on the quasi-invariance of the cross ratios, Israel J. Math. 30 (1978), 152-158.

[K4] _ Canonical mappings between Teichmüller spaces, Bull. Amer. Math. Soc. (N.S.) 4 (1981), 143-179.

[K5] _ On lifting Kleinian groups to $\mathrm{SL}(2, \mathbb{C})$, in Differential Geometry and Complex Analysis (I. Chavel and H. M. Farkas, eds.), Springer-Verlag, Berlin, Tokyo, 1985, pp. 181-193.

[K6] - Non-variational global coordinates for Teichmüller spaces, in Holomorphic Functions and Moduli II, Math. Sci. Res. Inst. Publ., vol. 11, Springer, New York, 1988, pp. 221-249.

[KM1] I. Kra and B. Maskit, The deformation space of a Kleinian group, Amer. J. Math. 103 (1981), 1065-1102.

[KM2] __ Bases for quadratic differentials, Comment. Math. Helv. 57 (1982), 603-626.

[Mn1] A. Marden, Geometrically finite Kleinian groups and their deformation spaces, Chapter 8 of Discrete Groups and Automorphic Functions (W. J. Harvey, ed.), Academic Press, London, 1977, pp. 259-293.

[Mn2] _ Geometric relations between homeomorphic Riemann surfaces, Bull. Amer. Math. Soc. (N.S.) 3 (1980), 1001-1017.

[Mn3] - Geometric complex coordinates for Teichmüller space, in Mathematical Aspects of String Theory (S. T. Yau, ed.), World Scientific, Singapore, 1987, pp. 341-364.

[Mt1] B. Maskit, Self-maps of Kleinian groups, Amer. J. Math. 93 (1971), 840-856.

[Mt2] _- Decomposition of certain Kleinian groups, Acta Math. 130 (1977), 63-82.

[Mt3] _ Moduli of marked Riemann surfaces, Bull. Amer. Math. Soc. 80 (1974), 773-777. 
[Mt4] _ Isomorphisms of function groups, J. Analyse Math. 32 (1977), 63-82.

[Mr] H. Masur, The extension of the Weil-Petersson metric to the boundary of Teichmüller spaces, Duke Math. J. 43 (1976), 623-635.

[Ms] J. P. Matelski, A compactness theorem for Fuchsian groups of the second kind, Duke Math. J. 43 (1976), 829-840.

[Ra] H. E. Rauch, Variational methods in the problem of moduli of Riemann surfaces, in Contributions to Function Theory, Tata Institute of Fundamental Research, Bombay, 1960, pp. 17-40.

[Ro] H. L. Royden, Automorphisms and isometries of Teichmüller space, in Advances in the Theory of Riemann Surfaces, Ann. of Math. Stud., no. 66, Princeton Univ. Press, Princeton, NJ, 1971, pp. 369-383.

[W1] S. A. Wolpert, On the homology of the moduli space of stable curves, Ann. of Math. (2) 118 (1983), 491-523.

[W2] _ The hyperbolic metric and the geometry of the universal curve (to appear).

Department of Mathematics, State University of New York at Stony Brook, Stony BROOK, NEW YORK 11794 